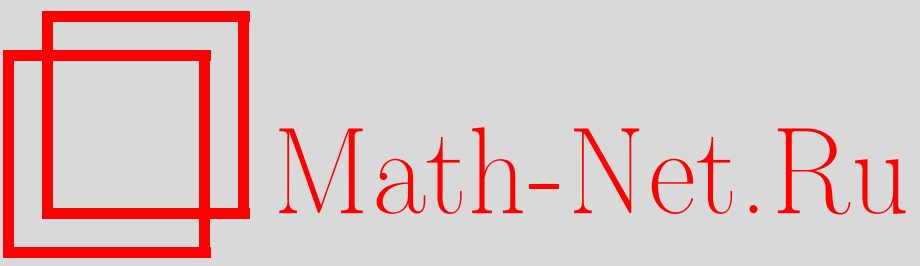

Р. Х. Зейтунян, Корректность задач динамики жидкостей (гидродинамическая точка зрения), УМН, 1999, том 54, выпуск 3, 3-92

DOI: https://doi.org/10.4213/rm152

Использование Общероссийского математического портала Math-Net.Ru подразумевает, что вы прочитали и согласны с пользовательским соглашением http: //www . mathnet.ru/rus/agreement

Параметры загрузки:

IP: 35.174 .16 .151

26 апреля 2023 г., 16:41:19 


\title{
КОРРЕКТНОСТЬ ЗАДАЧ ДИНАМИКИ ЖИДКОСТЕЙ (ГИДРОДИНАМИЧЕСКАЯ ТОЧКА ЗРЕНИЯ)
}

\author{
Р. Х. ЗЕЙтУнян \\ СОДЕРЖАНИЕ
}

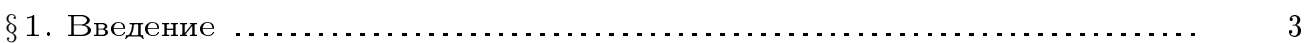

$\S 2$. Уравнения Навье-Стокса …....................................... 7

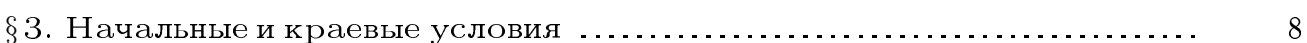

$\S 4$. Математическая структура задач гидродинамики ............... 10

$\S 5$. Сушествование и единственность ............................. 17

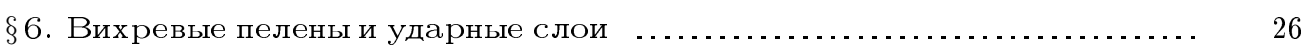

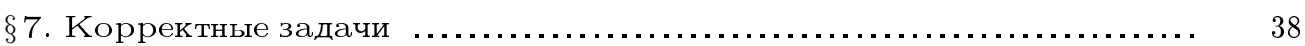

$\S 8$. Подход конечномерных динамических систем в задачах турбулент-

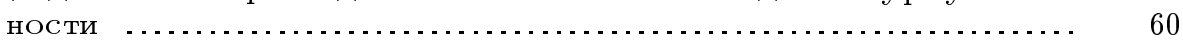

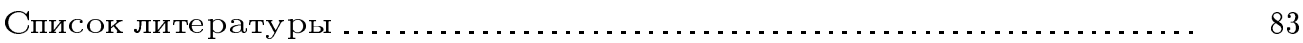

Доказательство существования, единственности, гладкости и устойчивости решений задач гидродинамики необходимо для того, чтобы придать смысл уравнениям и соответствуюшим начальньм и граничным условиям, которые управляют данными задачами.

При любом разумном выборе класса допустимых начальных данных гидродинамическая задача является корректно поставленной (в смысле Адамара [1]). Это означает, что (a) она имеет решение для любых начальных данных из этого класса; (b) peшение единственно для любых начальных условий; (c) решение непрерывно завиcum от начальных данных. В настоящей статье с гидродинамической точки зрения дается обзор некоторых аспектов задач о корректности, которые составляют очень трудньй и одновременно важньй раздел гидромеханики.

\section{$\S$ 1. Введение}

Приведенный (или вязкий) тензор напряжсений

$$
\boldsymbol{\Sigma}=\boldsymbol{T}+p \boldsymbol{I}
$$

Перевод статьи выполнен А. Ширикяном.

Работа выполнена при поддержке Российского фонда фундаментальных исследований (гранты № № 93-011-1450, 95-01074a). 
для так называемого течения Стокса в общем случае является функиией тензоpa деформаций $\boldsymbol{D}$; заметим, что когда жидкость находится в покое с нулевой скоростью изменения деформации, существуют нормальные компоненты $(-p)$ тензора напряжений, которые одинаковы во всех направлениях. Ньютоновская жидкость является фактически частным случаем жжидкости Стокса, когда в соотношении

$$
\boldsymbol{T}=-p \boldsymbol{I}+\boldsymbol{\Sigma}
$$

имеем

$$
\boldsymbol{\Sigma}=2 \mu \boldsymbol{D}+\lambda(\operatorname{div} \boldsymbol{u}) \boldsymbol{I}
$$

где в равенствах (1.1)-(1.3) мы полагаем $\boldsymbol{I}=\left(\delta_{i j}\right)$, а $\delta_{i i}=1$ и $\delta_{i j}=0$ при $j \neq i$. Отметим, что $\operatorname{div} \boldsymbol{u}=d_{k k}$ и $p-p^{*}=\left[\left(\lambda+\frac{2}{3} \mu\right)\right] d_{k k}$, где

$$
-p^{*}=\frac{1}{3} \operatorname{tr}(\boldsymbol{T})=\frac{1}{3}\left[T_{11}+T_{22}+T_{33}\right], \quad \boldsymbol{T}=\left(T_{i j}\right),
$$

причем $p^{*}$ можно интерпретировать как механическое давление жидкости, а $T_{i j}-($ декартовы) компоненты тензора напряжений $\boldsymbol{T}$.

В уравнении состояния (1.3) для ньютоновской жидкости скаляр $\lambda$ назьвается вторым коэффициентом вязкости, а $\mu_{v}=\lambda+\frac{2}{3} \mu$-обгемной вязкостью жидкости.

Для несжимаемой жидкости $\operatorname{div} \boldsymbol{u}=d_{k k}=0$, и поэтому скаляр $p$ из (1.2) есть просто механическое давление, которое совпадает с гидростатическим давлением, если жидкость находится в покое, и с гидродинамическим давлением, если она находится в движении (в этом случае мы получаем так называемые уравнения Навъе для несжимаемой, но вязкой жидкости). Для одноатомного газа $\mu_{v}=0$ и, следовательно, имеет место соотношение $\lambda=-\frac{2}{3} \mu$, которое назьвается равенством Стокса; таким образом, для одноатомного газа имеется только одна независимая вязкость. Для многоатомных газов и жидкостей объемная вязкость $\mu_{v}$ во многих случаях мало отличается от нуля, и приемлемой является аппроксимация

$$
p=p^{*}=-\frac{1}{3}\left[T_{11}+T_{22}+T_{33}\right]
$$

Во всяком случае, для жидкостей не имеет значения, равна нулю или нет вязкость $\mu_{v}$, так как всегда выполняется соотношение

$$
p=p^{*}
$$

Предположение о нњютоновости жидкости приводит к тому, что величины $\lambda$ и $\mu$ не зависят от скорости изменения тензора деформаций, и это явление наблюдается во многих часто встречающихся жидкостях и газах, таких как вода и воздух. Для нњютоновской жидкости предполагается также, что в уравнении энергии (записанной для внутренней энергии $E$, приходящей на единичную массу) тепловой поток (вектор) $\boldsymbol{Q}$ на единищу площади связан с температурой $T$ по закону теплопередачи Фурье:

$$
Q=-k \nabla T
$$


где $k$ - тепловой коэффициент диффузии жидкости. В уравнении энергии для нью-

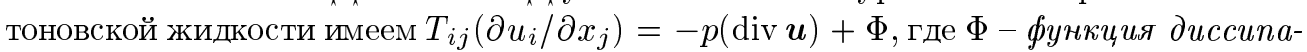
иии, представляющая собой скорость превращения механической энергии в тепловую. Функция $\Phi$ задается формулой

$$
\Phi=2 \mu d_{i j} d_{i j}+\lambda(\operatorname{div} \boldsymbol{u})^{2}
$$

которая показывает, что она совпадает со скоростью изменения работы вязких напряжений на единицу объема. Так как коэффициент $\lambda+\frac{2}{3} \mu=\mu_{v}$ объемной вязкости, так же как и $\mu$, положителен, функция $\Phi$ является на самом деле положительно определенной формой по $d_{i j}$, что видно из ее покомпонентной записи

$$
\begin{aligned}
\Phi=4 \mu\left[\left(d_{12}\right)^{2}+\left(d_{23}\right)^{2}+\right. & \left.\left(d_{31}\right)^{2}\right]+\left(\lambda+\frac{2}{3} \mu\right)\left[d_{11}+d_{22}+d_{33}\right]^{2} \\
& +\frac{2}{3} \mu\left[\left(d_{11}-d_{22}\right)^{2}+\left(d_{22}-d_{33}\right)^{2}+\left(d_{33}-d_{11}\right)^{2}\right]
\end{aligned}
$$

Отсюда ясно, какая часть диссипации возникает из-за недиагональных, или касательных, напряжений, и какая ее часть связана с диагональньми, или нормальными, компонентами вязких напряжений.

Наконеи, если $\sigma_{i j}-($ декартовы $)$ компоненты вязкого тензора $\boldsymbol{\Sigma}$, а $Q_{i}$ компоненты вектора теплового потока $Q$, то для ньютоновской жидкости имеют место следующие уравнения состояния:

$$
\begin{gathered}
T_{i j}=-p \delta_{i j}+\sigma_{i j} \\
\sigma_{i j}=\lambda(\operatorname{div} \boldsymbol{u}) \delta_{i j}+2 \mu d_{i j}, \quad d_{i j}=\frac{1}{2}\left[\frac{\partial u_{i}}{\partial x_{j}}+\frac{\partial u_{j}}{\partial x_{i}}\right] \\
Q_{i}=-k \frac{\partial T}{\partial x_{i}}
\end{gathered}
$$

где $i, j=1,2,3$.

Теперь важно заметить, что в классической механике сплошных сред из законов сохранения массы, линейного импульса и энергии выводится набор уравнений с частными производными относительно семи неизвестных функций, которыми являются давление $p$, плотность $\rho$, внутренняя энергия $E$, температура $T$ и компоненты скорости $u_{i}$ $(i=1,2,3)$. Предполагается, что коэффициенты вязкости $\lambda, \mu$ и тепловой коэффициент диффузии $k$ известны априори из экспериментальных данных; они могут быть постоянными или, более общо, заданными функциями от $T$ и $p$.

В случае ньютоновской жидкости, для которой справедливы соотношения (1.7), уравнение неразрывности (прямое следствие сохранения массы) и векторное уравнение движения (прямое следствие сохранения линейного импульса) образуют систему из четырех уравнений для $p$ (или $\rho$ ) и трех компонент скорости $u_{i}$. Для $E$ имеем уравнение энергии (которое является следствием первого закона термодинамики; при этом закон сохранения энергии получается, по существу, в результате его применения к элементу жидкости, находящейся в движении). 
Чтобы получить замкнутую систему уравнений для ньютоновской сжимаемой вязкой жидкости (так называемые уравнения Навье-Cтокса) относительно упомянутых выше семи неизвестных функций, необходимо добавить два уравнения состояния.

В настоящей статье мы в основном рассматриваем идеальный газ (воздух) и расширяемую жидкость (воду). Для идеального газа уравнения состояния имеют вид

$$
p=R \rho T \text { и } E=C_{v} T,
$$

где $R$ - константа, а $C_{v}$ - удельная теплоемкость при постоянном объеме. Для $р а с u и-$ ряемой жидкости уравнения состояния записьваются как

$$
\rho=\rho(T) \text { и } E=E(T),
$$

где $d E / d T=C(T)$ - удельная теплоемкость расширяемой жидкости, причем в рассматриваемом случае $\lambda=\lambda(T), \mu=\mu(T)$ и $k=k(T)$. Следует отметить, что если давление $p$ и коэффициенты $\mu$ и $\mu_{v}\left(=\lambda+\frac{2}{3} \mu\right)$ не зависят от температуры $T$, то уравнение состояния принимает вид

$$
p=P(\rho)
$$

в дальнейшем этот случай будем называть баротропным.

Однако для идеального газа (с константами $C_{p}$ и $C_{v}$ удельных теплоемкостей) уравнение состояния ( $p=R \rho T)$ можно записать в виде

$$
\frac{p}{\rho^{\gamma}} \exp \left(-\frac{S}{C_{v}}\right)=\mathrm{const}, \text { где } \gamma=\frac{C_{p}}{C_{v}},
$$

если ввести соответствующим образом энтропию $S$ идеального газа. Если энтропия остается постоянной при движсении потока, т.е. $S=S_{0}=$ const, то вместо (1.11) получаем баротропный поток с давлением

$$
p=\left[\exp \left(-\frac{S_{0}}{C_{v}}\right)\right] \rho \gamma, \quad \text { где } \gamma=\frac{C_{p}}{C_{v}},
$$

причем в этом случае функция $P(\rho)$ равна const $\rho \gamma$. Следовательно, в баротропном случае неизвестньми являются функции $\boldsymbol{u}, \rho$ и $p$. Случай

$$
\rho=\text { const }
$$

назьвается несжимаемым. Если же

$$
\frac{D \rho}{D t}=0
$$

то мы имеем дело с так называемым “изопикническим" (или изохорическим) случаем.

Книга Мейера [2] содержит очень полезное введение в математическую гидродинамику. Для глубокого изучения теоретических аспектов гидромеханики рекомендуется фундаментальная статья Серрина [3]. 


\section{§. Уравнения Навье-Стокса}

В механике сплошных сред (см., например, книгу Прагера [4]) из закона сохранения массы выводится следуюшее уравнение неразрывности:

$$
\frac{\partial \rho}{\partial t}+\operatorname{div}(\rho \boldsymbol{u})=0 \text { или } \frac{D \rho}{D t}+\rho \operatorname{div} \boldsymbol{u}=0
$$

где $D / D t=\partial / \partial t+(\boldsymbol{u} \cdot \nabla)-$ полная, или конвективная, производная, а декартовыми координатами градиента $\nabla$ являются $\partial / \partial x_{i}$. Аналогично, из принципа импульса (закон сохранения линейного импульса) выводится уравнение движсения:

$$
\rho \frac{D u_{i}}{D t}=\rho f_{i}+\frac{\partial T_{i j}}{\partial x_{j}}, \quad i=1,2,3 .
$$

Наконец, при условии отсутствия излучения сохранение энергии выражается первьм законом термодинамики, вследствие чего получаем уравнение әнергии:

$$
\rho \frac{D E}{D t}=T_{i j} d_{i j}-\frac{\partial Q_{j}}{\partial x_{j}} \text { или } T \rho \frac{D S}{D t}=-\operatorname{div} \boldsymbol{Q}+\Phi,
$$

где энтропия $S$ на единицу массы является параметром состояния, чья зависимость от других параметров состояния $(T, S, p$ и $\rho)$ выражается формулой $T d S=d E+p d(1 / \rho)$.

Принимая во внимание равенства (1.7) и (1.8), из уравнений (2.1)-(2.3) выводим следующую систему уравнений Навье-Стокса для идеального газа относительно неизвестных функций $u_{i}, p, \rho$ и $T$ :

$$
\begin{gathered}
\frac{D \rho}{D t}+\rho \frac{\partial u_{k}}{\partial x_{k}}=0 \\
\rho \frac{D u_{i}}{D t}+\frac{\partial p}{\partial x_{i}}=\rho f_{i}+\frac{\partial}{\partial x_{i}}\left[\lambda \frac{\partial u_{k}}{\partial x_{k}}\right]+\frac{\partial}{\partial x_{j}}\left[\mu \frac{\partial u_{i}}{\partial x_{j}}+\frac{\partial u_{j}}{\partial x_{i}}\right] \\
\rho C_{p} \frac{D T}{D t}=\frac{D p}{D t}+\Phi+\frac{\partial}{\partial x_{i}}\left[k \frac{\partial T}{\partial x_{i}}\right] \\
p=R \rho T
\end{gathered}
$$

где $C_{p}$ - удельная теплоемкость при постоянном давлении, $R=C_{p}-C_{v}$, а $\Phi$ задается равенством (1.6) с $d_{i j}=\frac{1}{2}\left[\partial u_{i} / \partial x_{j}+\partial u_{j} / \partial x_{i}\right]$.

Принимая во внимание уравнения состояния (1.9), для расширяемой жидкости из (2.1)-(2.3) вьводятся следующие уравнения Навье-Стокса относительно неизвестных функций $u_{i}, p$ и $T$ :

$$
\begin{gathered}
\frac{D(\log \rho)}{D t}+\operatorname{div} \boldsymbol{u}=0 \\
\rho(T) \frac{D u_{i}}{D t}+\frac{\partial p}{\partial x_{i}}=\rho f_{i}-\frac{\partial}{\partial x_{i}}\left[\lambda(T) \frac{D(\log \rho)}{D t}\right] \\
+\frac{\partial}{\partial x_{j}}\left[\mu(T)\left(\frac{\partial u_{i}}{\partial x_{j}}+\frac{\partial x_{j}}{\partial x_{i}}\right)\right], \\
\rho C(T) \frac{D T}{D t}-p \frac{D(\log \rho)}{D t}=\frac{\partial}{\partial x_{i}}\left[k(T) \frac{\partial T}{\partial x_{i}}\right]+\lambda(T)\left[\frac{D(\log \rho)}{D t}\right]^{2} \\
+\frac{\mu(T)}{2}\left[\frac{\partial u_{i}}{\partial x_{j}}+\frac{\partial u_{j}}{\partial x_{i}}\right]^{2},
\end{gathered}
$$


где предполагается, что коэффициент $\rho=\rho(T)$, так же как и $\lambda(T), \mu(T), k(T)$ и $C(T)$, является априори известной функцией температуры $T$. Обычно функция $\rho$ имеет вид $\rho=\rho\left(T_{0}\right)\left[1-\alpha\left(T-T_{0}\right)\right]$, где $\alpha$ - постоянньй коэффициент объемного расширения, причем для типичных жидкостей, используемых в экспериментах, имеем $\alpha \approx 5 \cdot 10^{-4} K^{-1}$.

В случае так назьваемой задачи тепловой неустойчивости Рэлея-Бенара для покояшейся расширяемой жидкости, находящейся между двумя бесконечньми горизонтальными плоскостями с различньми постоянньми температурами $\left(T=T_{0}\right.$ на верхней плоскости и $T=T_{0}+\Delta T_{0}$ на нижней), мы можем ввести отклоненную температуру $\theta=\left(T-T_{0}\right) / \Delta T_{0}\left(\Delta T_{0}>0\right)$ и написать следуюшее соотношение между $\rho / \rho_{0}$ и $\theta$ :

$$
\frac{\rho}{\rho_{0}}=1-\beta \theta
$$

где $\rho_{0}=\rho\left(T_{0}\right)$, а $\beta=\alpha \Delta T_{0}$. Если $\beta \ll 1$, то асимптотически можно строго вьвести так называемые приближенные уравнения Буссинеска для расшшряемой жидкости (см. Зейтунян [5]).

\section{§. Начальные и краевые условия}

Для уравнений Навье-Стокса (2.4) можно задавать различные начальные и краевые условия. Чаше всего встречаются следующие условия.

Уравнения $(2.4 \mathrm{a}, \mathrm{b}, \mathrm{c})$ являются эволюционными уравнениями относительно функций $\rho, u_{i}(i=1,2,3)$ и $T$. Для определения $\rho, u_{i}$ и $T$ надо рассматривать задачу Коши. Поэтому задаются следующие начальные условия:

$$
\boldsymbol{u}=\boldsymbol{u}^{0}(\boldsymbol{x}), \quad \rho=\rho^{0}(\boldsymbol{x}), \quad T=T_{0}(\boldsymbol{x}) \text { при } t=0,
$$

где $\rho^{0}(\boldsymbol{x})>0, T^{0}(\boldsymbol{x})>0$ и $\boldsymbol{u}^{0}(\boldsymbol{x})$ - заданные значения плотности, температуры и вектора скорости. Более того, при рассмотрении задачи со свободной границей надо задать начальное условие для границы $\partial \Omega(t)$. Очевидно, $\rho$ и $T$ можно заменить на любую другую пару независимых параметров состояния.

Некоторые краевые условия можно налагать для разных физических ситуаций. Рассмотрим в качестве примера движение жидкости в недеформируемом резервуаре $\Omega$ (с не зависящей от времени $t$ границей $\partial \Omega$ ), которьй представляет собой связное открытое подмножество в $\mathbb{R}^{d}$ (где $d \geqslant 1$ - физическая размерность). Разная структура уравнений приводит к необходимости различать вязкие и невязкие жидкости.

Вязкие жидкости: $\mu>0, \mu_{v} \geqslant 0$.

В этом случае физические эффекты, вызванные присутствием динамического коэффициента вязкости $\mu$ (коэффициент сдвига), приводят к условию непроскальзывания:

$$
\boldsymbol{u}=0 \text { на } \partial \Omega \text {. }
$$

Объемно-вязкие жмидкости: $\mu=0, \mu_{v}>0$.

Так как отличен от нуля только коэффициент объемной вязкости $\mu_{v}$, то в рассматриваемой ситуации на границе налагается условие проскальзывания:

$$
\boldsymbol{u} \cdot \boldsymbol{n}=0 \text { на } \partial \Omega \text {. }
$$


Здесь и ниже через $\boldsymbol{n}=\boldsymbol{n}(\boldsymbol{x})$ обозначается единичная внешшяя нормаль к $\partial \Omega$.

Невязкие жидкости: $\mu=0, \mu_{v}=0$.

В этом случае также налагается краевое условие проскальзьвания (3.3).

Что касается (абсолютной) температуры $T$, граничные условия для нее отличаются в следуюших двух взаимоисключающих случаях: $k>0$ и $k=0$.

Проводящие жицжости: $k>0$.

Физический смысл имеют несколько граничных условий. Мы ограничимся следующими тремя условиями, которые являются наиболее распространенными:

$$
\begin{gathered}
T=T_{w} \text { на } \partial \Omega \text { (Дирихле), } \\
k \partial T / \partial n=\boldsymbol{\Xi} \text { на } \partial \Omega \text { (Нейман), } \\
k \partial T / \partial n+h\left(T-T_{0}\right)=\boldsymbol{\Xi} \text { на } \partial \Omega \text { (третий тип условий), }
\end{gathered}
$$

где $T_{w}>0$ и $\boldsymbol{\Xi}$ - известные функции, а $h>0$ - заданная константа.

Непроводящие жидкости: $k=0$.

Если выполнено (3.2) или (3.3), то нет необходимости налагать граничное условие для температуры $T$, так как в этих случаях она не переносится через гранищу.

Мы ограничимся, в основном, обсуждением поля скоростей и плотности, так как условия (3.4)-(3.6) для абсолютной температуры $T$ обшеизвестны.

Прежде всего отметим, что во многих ситуациях (задачи втекания и вытекания) на $\partial \Omega$ нельзя налагать условие обращения скорости в нуль. Так обстоит дело, например, в случае течения вблизи профиля, когда областью втекания служит естественньй верхний бьеф (а область вытекания находится в спутном следе), или в случае течения вблизи твердого тела, когда условие обрашения скорости в нуль можно накладьвать только на границе тела. В указанных случаях могут налагаться разные граничные условия. Рассмотрим сначала вязкий случай. Для поля скоростей можно задать (неоднородное) условие Дирихле всюду на границе или только на области втекания, т.е. на подмножестве $\partial \Omega$, где $\boldsymbol{u} \cdot \boldsymbol{n}<0$, тогда как на остальной части налагаются условия

$$
\boldsymbol{u} \cdot \boldsymbol{n}=U^{+} \geqslant 0, \quad(\boldsymbol{n} \cdot \boldsymbol{D}) \cdot \boldsymbol{t}=0,
$$

где $\boldsymbol{t}$ - единичньй касательньй вектор к $\partial \Omega$, а $\boldsymbol{D}$ - скорость изменения тензора деформаций. Более того, заметим, что условия

$$
\boldsymbol{u} \cdot \boldsymbol{n}=0, \quad(\boldsymbol{n} \cdot \boldsymbol{D}) \cdot \boldsymbol{t}=0
$$

также можно задавать на всей гранище $\partial \Omega$, однако в этом случае не будет областей втекания и вытекания.

Более важным является анализ краевого условия для плотности $\rho$, так как оказывается, что его необходимо задать на области втекания. В самом деле, гиперболическое уравнение первого порядка (2.1а) можно решить методом характеристик, а граничное значение $\rho$ на области втекания есть просто начальное условие (данное Коши) для плотности, заданное на нехарактеристической поверхности. Заметим, что если коэффициент теплопроводности $k$ равен нулю, то для температуры $T$ на области втекания надо задать такое же граничное условие Дирихле, так как в этом случае уравнение (2.4c) также является гиперболическим. 
Более сложньм является случай невязкой (эйлеровой) жидкости $(\mu=\lambda=k=0)$. В самом деле, в этом случае уравнения (2.4a)-(2.4d) составляют гиперболическую систему первого порядка, и число краевых условий различно для дозвукового $(|\boldsymbol{u}|<a)$ и сверхзвукового $(|\boldsymbol{u}|>a)$ потоков, где $a=[\gamma R T]^{1 / 2}$ - локальная скорость звука для идеального газа. Рассмотрим, например, случай $d=3$. Анализируя знаки собственных значений ассоциированной характеристической матрицы, приходим к выводу, что в зависимости от того, является ли жидкость сверхзвуковой или дозвуковой, необходимо налагать пять или четыре граничных условия на области втекания. При этом на области вытекания в сверхзвуковом случае не задается никаких условий, а в дозвуковом случае налагается одно условие.

Мы не будем обсуждать этот вопрос более подробно, а рассмотрим только случай, когда невязкая жидкость удовлетворяет условию проскальзывания (3.3), причем граница является характеристической поверхностью.

Дальнейшие сведения о граничных задачах со втеканием и вытеканием для несжимаемой системы уравнений Навье-Стокса и невязких уравнений Эйлера можно найти в статьях Густафсона, Сандстрёма [6] и Олигера, Сандстрёма [7]. Другой интересньй набор граничных условий возникает при рассмотрении задачи со свободной границей, т.е. задачи, в которой жидкость не содержится в фиксированной области и может свободно двигаться. В этом случае вектор $\boldsymbol{n} \cdot T$ задается на (межфазной границе) $\partial \Omega$, причем требуется, чтобы скалярное произведение $\boldsymbol{u} \cdot \boldsymbol{n}$ либо обрашалось в нуль (стационарный случай), либо было равно нормальной компоненте скорости самой границы (нестационарный случай). Значение вектора $\boldsymbol{n} \cdot T$ на межфазной границе может либо равняться нулю (свободное расширение жидкости в вакууме), либо совпадать с выражением

$$
-p_{e} \boldsymbol{n}+2 \sigma K \boldsymbol{n}+\nabla_{S} \sigma
$$

где $p_{e}$ - внешнее давление, $\sigma$ - поверхностное натяжение (зависяшее от температуры в случае, когда жидкость является расширяемой), $K$ - средняя кривизна границы, а $\nabla_{S}=\nabla-\boldsymbol{n}(\boldsymbol{n} \cdot \nabla)$ - поверхностный (проектированный) градиент на границе. Но в этом случае необходимо также налагать условие теплопередачи через межфазную границу:

$$
k \frac{\partial T}{\partial n}+h_{S} T=\text { заданная функция, }
$$

где (постоянный) коэффициент теплопередачи $h_{S}$ иногда называют иислом Био. Так как $d \sigma / d T \neq 0$, то в случае задачи о "пленке" необходимо учитьвать число Ма-

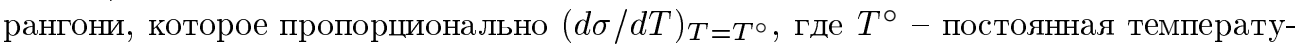
ра (причем в этом случае мы рассматриваем задачу со свободной границей Бенара-Марангони для тонкой пленки; см. Зейтунян [8]).

Естественно, мы налагаем также условие на межфазную границу $\partial \Omega$, так как она является неизвестной задачи. В нестационарном случае задается также начальное условие для границы.

\section{$\S$ 4. Математическая структура задач гидродинамики}

Для простоты мы всегда будем предполагать, что уравнение (2.4c) для температуры $T$ можно отделить от двух других уравнений (2.4a) и (2.4b), т.е. мы имеем дело с 
баротропной жидкостью (с постоянной энтропией). Более того, мы будем рассматривать только задачи в ограниченной области $\Omega$.

Грубо говоря, можно утверждать, что уравнения для вязкой жидкости являются параболическими, а уравнения для невязкой жидкости (уравнения Эйлера) - гиперболическими. Однако более детальньй анализ структуры уравнений (2.4a), (2.4b) показьвает, что это не совсем так. В самом деле, уравнение неразрывности (2.4a) относительно $\rho$ является гиперболическим, несмотря на присутствие вязкости.

Таким образом, более точно можно сказать, что система уравнений Навье-Стокса является гиперболическо-параболической или неполно-параболической в смысле определения, предложенного в работах Белова, Яненко [9] и Стрикверда [10], посвяшенных изучению математических свойств этих уравнений.

Термин несжимаемое движение означает такое течение жидкости, при котором $\rho \equiv$ const. Таким образом, несжимаемость в гидродинамике является свойством не жидкости, а представления, с помощью которого аппроксимируется ее реальное движение. Менее ограничительным является определение, согласно которому несжимаемость означает, что функция $\rho(\boldsymbol{x}(\boldsymbol{a}, t), t)$ зависит только от лагранжевой метки $\boldsymbol{a}$.

В обоих случаях

$$
\frac{D \rho}{D t}=0,
$$

так что из (2.1а) следует равенство

$$
\operatorname{div} \boldsymbol{u}=0 .
$$

В несжимаемом случае давление не связано с термодинамическими неизвестными, и вместо уравнения состояния (1.8) требуется, чтобы любая заданная часть жидкости сохраняла свой объем при движении; как хорошо известно, выполнение этого требования обеспечивается уравнением (4.2). В результате уравнения (2.4a) и (2.4b) принимают вид

$$
\begin{gathered}
\frac{D \rho}{D t}=0 \\
\rho \frac{D u_{i}}{D t}+\frac{\partial p}{\partial x_{i}}=\rho f_{i}+\frac{\partial}{\partial x_{j}}\left[\mu\left(\frac{\partial u_{i}}{\partial x_{j}}+\frac{\partial u_{j}}{\partial x_{i}}\right)\right],
\end{gathered}
$$

где $p$ - неизвестная функция (в некотором смысле, множитель Лагранжа, связанный с условием $\operatorname{div} \boldsymbol{u}=0$ ).

Заметим, что решение $\rho(t, \boldsymbol{x})$ уравнения

$$
\frac{D \rho}{D t}=0 \text { с начальным условием } \rho=\rho^{0}(\boldsymbol{x}),
$$

где поле скоростей $\boldsymbol{u}(t, \boldsymbol{x})$ рассматривается как заданный вектор, удовлетворяюший граничному условию (4.2) или (4.3) и уравнению $\operatorname{div} \boldsymbol{u}=0$, задается известной формулой

$$
\rho(t, \boldsymbol{x})=\rho^{0}(\boldsymbol{U}(0, t, \boldsymbol{x})),
$$


где характеристическое направление $\boldsymbol{U}(s, t, \boldsymbol{x})$ является решением системы обыкновенных уравнений

$$
\frac{\partial}{\partial t}[\boldsymbol{U}(s, t, \boldsymbol{x})]=\boldsymbol{u}(t, \boldsymbol{U}(s, t, \boldsymbol{x})), \quad \boldsymbol{U}(s, s, \boldsymbol{x})=\boldsymbol{x}
$$

функция $\boldsymbol{U}(s, t, \boldsymbol{x})$ назьвается также потоком векторного поля $\boldsymbol{u}(t, \boldsymbol{x})$.

Непосредственно из формулы (4.5) следует, что

$$
0<\inf _{\Omega} \rho^{0} \leqslant \rho(t, x) \leqslant \sup _{\Omega} \rho^{0}
$$

Этот факт имеет несколько следствий: во-первых, коэффициент при производной по времени функции $u_{i}$ в уравнении $(4.3 \mathrm{~b})$ не обрашается в нуль, т.е. $(4.3 \mathrm{~b})$ не может вырождаться; во-вторых, если $\rho^{0}(\boldsymbol{x})=\rho^{*}$ - положительная константа, то решением начальной задачи (4.4) является функция $\rho(t, \boldsymbol{x}) \equiv \rho^{*}$, а уравнение $(4.3 \mathrm{~b})$ принимает вид

$$
\frac{\partial \boldsymbol{u}}{\partial t}+\boldsymbol{u} \cdot \nabla \boldsymbol{u}-f=-\nabla p^{*}+\nu^{*} \Delta \boldsymbol{u}
$$

где $p^{*}=p / \rho^{*}$, а $v^{*}=\mu\left(\rho^{*}\right) / \rho^{*}$.

Уравнения (4.2) и (4.7) обычно назьваются уравнениями Навье (если $\left.\nu^{*}>0\right)$ или Эйлера (если $\nu^{*}=0$ ) для однородной несжимаемой жидкости, тогда как (4.1), (4.2) и (4.3) назьваются уравнениями Навье или Эйлера для неоднородной несжимаемой жидкости.

Мы не будем обсуждать математическую теорию этих уравнений, которые подробно изучались в литературе; заинтересованньй читатель может обратиться к следующим книгам: Ладыженская [11], Шинброт [12], Темам [13], Антонцев, Кажихов, Монахов [14], Константин, Фойаш [15], фон Валь [16], Майда [17].

В сжимаемом случае из уравнения $(2.1)$ с начальным условием $\rho=\rho^{0}(\boldsymbol{x})$ при $t=0$ находим

$$
\rho(t, \boldsymbol{x})=\rho^{0}(\boldsymbol{U}(0, t, \boldsymbol{x})) \exp \left[-\int_{0}^{t}(\operatorname{div} \boldsymbol{u})(s, \boldsymbol{U}(s, t, \boldsymbol{x})) d s\right]
$$

следовательно, $\rho$ может вырождаться в конечный момент времени $t^{*}$ в точке $\boldsymbol{x}$, если

$$
\left|\int_{0}^{t^{*}}(\operatorname{div} \boldsymbol{u})\left(s, \boldsymbol{U}\left(s, t^{*}, \boldsymbol{x}\right)\right) d s\right|=+\infty .
$$

Можно сказать, что одним из главных математических задач, касающихся сжимаемых жидкостей, является вывод априорных оценок, которые обеспечивают невырождаемость плотности $\rho$. Очевидно, это легче установить локально по времени или для малых начальных данных (см., например, Валли [18]).

Дифференциальное уравнение

$$
\frac{\partial U}{\partial t}+U \frac{\partial U}{\partial x}=0
$$


для функции $U(t, x)$ часто изучается как упрошенная модель для уравнений невязкой (эйлеровой) гидродинамики. Его решения могут иметь на конечных временах разрывы типа ударных волн. Для исследования таких решений приведенное выше уравнение перепишем в виде закона сохранения:

$$
\frac{\partial U}{\partial t}+\frac{\partial\left[\frac{1}{2} U^{2}\right]}{\partial x}=0
$$

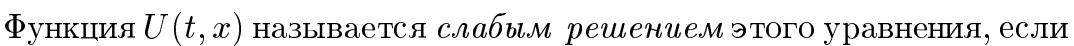

$$
\iint\left\{U \frac{\partial \varphi}{\partial t}+\left[\frac{1}{2} U^{2}\right] \frac{\partial \varphi}{\partial x}\right\} d t d x=0
$$

для любой ( пробной) функции $\varphi(t, x)$, которая непрерывно дифференцируема при всех $t, x$ и тождественно равна нулю вне некоторой конечной области на плоскости $(t, x)$ (т.е. $\varphi$ имеет компактньй носитель).

При этом функция $U(t, x)$ такова, что ее обобщенная производная по времени и обобщенная производная ее квадрата $U^{2}$ по $x$ связаны уравнением (4.9). Известно, что слабые решения соответствующих законов сохранения в гидродинамике имеют физический смысл. В частности, их конечные скачки удовлетворяют так называемому условию скачка Ренкина-Гюгонио для гидродинамических ударных волн. Однако правильную форму закона сохранения следует определить из физических соображений. Вместо (4.9) мы можем рассматривать уравнение

$$
\frac{\partial\left[\frac{1}{2} U^{2}\right]}{\partial t}+\frac{\partial\left[\frac{1}{3} U^{3}\right]}{\partial x}=0
$$

однако его решения не совпадают с решениями уравнения (4.9)! Из математических соображений невозможно понять, какие слабые решения следует назьвать обобщенными решениями исходного уравнения. Слабое решение уравнения (4.9) может совпадать со слабьм решением уравнения (4.11) до появления ударной волны (т.е. до тех пор пока они являются сильными решениями), а затем отличаться от него.

Рассмотрим теперь нестационарное одномерное движение (идеальной) невязкой жидкости при условии, что теплопроводностью можно пренебречь. В качестве модели можно представить себе жидкость, которая течет без трения по длинной трубе с поперечным сечением единичной плошади. Обозначим через $\rho, u, p$ и $e$ соответственно плотность, скорость, давление и внутреннюю энергию на единицу массы жидкости. Каждая из этих величин является функцией переменных $x$ и $t$, где $x$ - декартова координата вдоль трубы, а $t$ - время.

В этом случае, полагая

$$
U=\left(\begin{array}{c}
\rho \\
\rho u \\
e
\end{array}\right), \quad F=\left(\begin{array}{c}
\rho u \\
\left(u^{2} \rho\right)+p \\
(e+p) u
\end{array}\right)
$$

мы можем переписать уравнения Эйлера в векторном виде

$$
\frac{\partial U}{\partial t}+\frac{\partial F}{\partial x}=0
$$


Заметим, что пока неизвестных функций (это $\rho, \rho u, e, p)$ больше, чем уравнений. Однако, согласно законам термодинамики существует функциональная зависимость (уравнение состояния) между $p, \rho$ и $е$. Для идеального газа она имеет вид

$$
p=(\gamma-1) \rho e .
$$

Если под символом $p$ в (4.12) понимать это выражение, то все компоненты $F$ будут функциями от компонент вектора $U$. Следовательно, мы можем записать $F=F(U)$ и поэтому

$$
\frac{\partial U}{\partial t}+\frac{\partial F(U)}{\partial x}=0
$$

Система уравнений такого вида с произвольным числом неизвестных и независимых переменных называется системой законов сохранения. Обобщение на случай нескольких пространственных переменных очевидно.

Любую систему законов сохранения можно переписать в квазилинейной форме при исследовании гладких решений. Для этого определим матрицу $A=A(U)$ по формуле

$$
A_{j k}=\frac{\partial F_{j}(U)}{\partial U_{k}}
$$

и запишем систему (4.14) в виде

$$
\frac{\partial U}{\partial t}+A(U) \frac{\partial U}{\partial x}=0
$$

Если $W(t, x)$ - векторнозначная пробная функция (с таким же числом компонент, что и $U$ ), то по определению обобшенной производной (компоненты вектор-функций $U(t, x)$ и $F(t, x)$ рассматриваются как распределения на плоскости $(t, x)$, а производные, фигурируюшие в (4.14), понимаются в обобшенном смысле) уравнение (4.14) означает, что

$$
\iint_{-\infty}^{+\infty}\left[\frac{\partial W}{\partial t} U+\frac{\partial W}{\partial x} F(U)\right] d x d t=0
$$

Любая функция $U(t, x)$, удовлетворяющая уравнению (4.16) для произвольной пробной вектор-функции $W(t, x)$, называется слабъм решением системы законов сохранения (4.14).

Наконец, мы можем определить слабое решение эволющионных уравнений Навъе. Для этого естественно ввести функциональное пространство $H$ бездивергентных вектор-функций в области $\Omega$, которые обрашаются в нуль на $\partial \Omega$.

Скалярное произведение в $H$ будем обозначать скобками:

$$
(\boldsymbol{f}, \boldsymbol{g})=\int_{\Omega} \boldsymbol{f} \cdot \boldsymbol{g} d \boldsymbol{x} .
$$

Рассмотрим следуюшую әволюционную задачу Навье для функций $\boldsymbol{u}$ и $\pi$ :

$$
\begin{gathered}
\frac{\partial \boldsymbol{u}}{\partial t}+\boldsymbol{u} \cdot \nabla \boldsymbol{u}+\nabla \pi=\nu_{0} \Delta \boldsymbol{u} \text { в } \Omega, \quad \boldsymbol{u}=\boldsymbol{u}^{0} \text { при } t=0 ; \\
\nabla \cdot \boldsymbol{u}=0 \text { в } \Omega, \quad \boldsymbol{u}=0 \text { на } \partial \Omega .
\end{gathered}
$$


Пусть $\boldsymbol{u}$ - классическое решение начальной задачи Навье (4.18) (в этом случае все слагаемые в уравнениях Навье корректно определены). Пусть $f \in H$ - функция с компактным носителем. Умножим систему уравнений Навье на $f$ и проинтегрируем по $\Omega$.

Прежде всего заметим, что слагаемое $\int_{\Omega} \nabla \pi \cdot f d \boldsymbol{x}$ равно нулю (см. [12; с. 157]). Интегрируя по частям и дважды применяя формулу Грина, вместо уравнений Навье получим

$$
\int_{\Omega}\left[\boldsymbol{u} \cdot \frac{\partial \boldsymbol{f}}{\partial t}+(\boldsymbol{u} \cdot \nabla) \boldsymbol{f} \cdot \boldsymbol{u}+\nu_{0} \boldsymbol{u} \cdot \Delta \boldsymbol{f}\right] d \boldsymbol{x}=\frac{\partial}{\partial t}\left(\int_{\Omega} \boldsymbol{u} \cdot \boldsymbol{f} d \boldsymbol{x}\right) .
$$

Это уравнение можно интегрировать по $t$, что приводит к соотношению

$$
\int_{0}^{\infty}\left[\left(\boldsymbol{u}, \frac{\partial \boldsymbol{f}}{\partial t}\right)+(\boldsymbol{u} \cdot \nabla \boldsymbol{f}, \boldsymbol{u})+\nu_{0}(\boldsymbol{u}, \Delta \boldsymbol{f})\right] d t=-\left(\boldsymbol{u}^{0}, \boldsymbol{f}_{t=0}\right)
$$

Мы использовали финитность функции $\boldsymbol{f} \in H$ и начальное условие для $\boldsymbol{u}$.

Мы установили, что любое классическое решение начальной задачи Навье (4.18) удовлетворяет равенству (4.20) для произвольного $f \in H$. С другой стороны, легко представить себе функцию $\boldsymbol{u}$, удовлетворяющую тождеству (4.20), которая не обладает достаточной гладкостью, чтобы уравнения Навье (4.18a) имели смысл! Функция $\boldsymbol{u}$ называется слабым решением начальной задачи Навье (4.18a), если она принадлежит пространству Соболева $W^{2,2}\left(\Omega^{\prime}\right), \Omega^{\prime} \subset \Omega$, и удовлетворяет равенству (4.20) для всех $f \in H$ (точное определение можно найти в [13; гл. $3, \S 3$, п. 3.6]). Легко показать, что

достаточно гладкое слабое решение является классическим решением, если граница $\Omega$ также является гладкой.

Линейная система

$$
\frac{\partial U}{\partial t}+A(t, x) \frac{\partial U}{\partial x}=0
$$

с переменными коэффициентами называется гиперболической в области $R$ плоскости $(t, x)$, если матрица $A(t, x)$ имеет вещественные собственные значения и полную систему собственных функций в любой точке области $R$.

Для нелинейной системы гиперболичность зависит не только от уравнения, но и от решения. Пусть $U(t, x)$ - решение системы (4.15). Тогда система назьвается гиперболической в области $R$ для решения $U(t, x)$, если матрица $A(U(t, x))$ обладает сформулированными вьше свойствами; другими словами, система, полученная линеаризацией (4.15) на решении $U(t, x)$, является гиперболической в $R$. Часто налагаются ограничения на зависимость $A(U)$ от $U$ или $A(U(t, x))$ от $(t, x)$; например, предполагается, что эти функции удовлетворяют условию Лишшица. Более того, чтобы избежать плохой обусловленности матриц, часто требуют

$$
\text { ограниченности произведения }\|T\| \cdot\left\|T^{-1}\right\| \text { в области } R,
$$

где $T$ - матрица, диагонализируюшая $A$ :

$$
T A T^{-1}=D .
$$


Применяя матрицу $T$ непосредственно к системе (4.15), получим

$$
\sum_{k=1}^{l} T_{j k}(U)\left[\frac{\partial}{\partial t}+\lambda_{j}(U) \frac{\partial}{\partial x}\right] U_{k}=0 \quad(j=1,2, \ldots, l),
$$

где $\lambda_{j}$ - собственные значения матрицы $A(U)$. Эти уравнения представляют собой характеристическую форму системы (4.15).

Система является гиперболической тогда и только тогда, когда она может быть преобразована в систему вещественных уравнений, имеющую характеристическую форму.

Самьй простой способ приведения уравнений одномерной (эйлеровой) гидродинамики к характеристической форме состоит в выборе плотности $\rho$, скорости $u$ и удельной энтропии $S$ в качестве зависимых переменных. Если уравнение состояния записано как

$$
p=p(S, \rho), \quad c^{2}=\frac{\partial}{\partial \rho}[p(S, \rho)]
$$

то уравнения Эйлера принимают вид

$$
\begin{gathered}
\left(\frac{\partial}{\partial t}+u \frac{\partial}{\partial x}\right) \rho+\rho \frac{\partial u}{\partial x}=0 \\
\left(\frac{\partial}{\partial t}+u \frac{\partial}{\partial x}\right) u+\frac{1}{\rho}\left(c^{2} \frac{\partial \rho}{\partial x}+\frac{\partial p}{\partial S} \frac{\partial S}{\partial x}\right)=0 \\
\left(\frac{\partial}{\partial t}+u \frac{\partial}{\partial x}\right) S=0
\end{gathered}
$$

Матрища $A$ задается формулой

$$
A=\left(\begin{array}{ccc}
u & \rho & 0 \\
\frac{c^{2}}{\rho} & u & \frac{1}{\rho} \frac{\partial}{\partial S}[p(S, \rho)] \\
0 & 0 & u
\end{array}\right),
$$

а ее собственные значения $\lambda_{1}, \lambda_{2}, \lambda_{3}$ являются корнями характеристического уравнения

$$
\operatorname{det}[A-\lambda I]=\left[(u-\lambda)^{2}-c^{2}\right](u-\lambda)=0
$$

и, следовательно, равны $\lambda=u \pm c$ и $\lambda=u$.

Характеристиками являются траектории звуковых сигналов, распространяюшихся вперед и назад, и траектории частиц жидкости. Характеристическая форма уравнений легко находится:

$$
\begin{aligned}
& {\left[\frac{\partial}{\partial t}+(u+c) \frac{\partial}{\partial x}\right] p+\rho c\left[\frac{\partial}{\partial t}+(u+c) \frac{\partial}{\partial x}\right] u=0} \\
& {\left[\frac{\partial}{\partial t}+(u-c) \frac{\partial}{\partial x}\right] p-\rho c\left[\frac{\partial}{\partial t}+(u-c) \frac{\partial}{\partial x}\right] u=0} \\
& \left(\frac{\partial}{\partial t}+u \frac{\partial}{\partial x}\right) S=0
\end{aligned}
$$


Важным частным случаем является ситуация, когда әнтропия постоянна в начальныц й момент времени $t=0$, т.е. $S(0, x)$ не зависит от $x$. В этом случае третье уравнение в (4.25) показывает, что энтропия $S$ остается постоянной (до тех пор, пока не возникают ударные волны), т.е. течение является изәнтропическим. При этом функции $p$ и $c$ зависят только от $\rho$ и временно обозначаются через $p(\rho)$ и $c(\rho)$.

Если обозначить через $\sigma(\rho)$ термодинамическую величину

$$
\sigma=\sigma(\rho)=\int \frac{1}{\rho c(\rho)} d p(\rho),
$$

то первые два уравнения после деления на $\rho c$ примут вид

$$
\left[\frac{\partial}{\partial t}+(u \pm c) \frac{\partial}{\partial x}\right](\sigma \pm u)=0 .
$$

Выражения $\sigma \pm u$, называемые инвариантами $Р$ иман $a$, постоянны вдоль прямых и обратных характеристик. Для газов, удовлетворяюших $\gamma$-закону, имеем $\sigma=2 c /(\gamma-1)$, и поэтому в качестве зависимых переменных можно выбрать $u(t, x)$ и $c(t, x)$.

Двумерные уравнения Эйлера в характеристической форме имеют вид (см., например, книгу Рихтмайера [19; том I])

$$
\begin{gathered}
\frac{D p}{D t}-c^{2} \frac{D \rho}{D t}=0 \\
\mu \cdot\left(\frac{D \boldsymbol{u}}{D t}+\frac{1}{\rho} \nabla p\right)=0 \\
\rho c\left(\lambda \frac{D}{D t}+c \nabla\right) \cdot \boldsymbol{u}+\left(\frac{D}{D t}+c \lambda \cdot \nabla\right) p=0 .
\end{gathered}
$$

Все векторы в уравнениях (4.27) имеют по две компоненты; в частности, $\nabla=(\partial / \partial x, \partial / \partial y)$, а $\lambda$ и $\mu$ - произвольные единичные векторы в плоскости $(x, y)$; $D / D t=\partial / \partial t+\boldsymbol{u} \cdot \nabla=\partial / \partial t+u \partial / \partial x+v \partial / \partial y-$ оператор дифференцирования вдоль траекторий частиц, а $c=[\gamma p / \rho]^{1 / 2}$ - адиабатическая скорость звука. Уравнения (4.27) обобшают систему (4.25).

\section{$\S 5$. Существование и единственность}

Одной из фундаментальных проблем, возникающих при исследовании задач математической физики, является вопрос корректности, т.е. сушествуют ли решения на самом деле и определены ли они однозначно. Ответом на этот вопрос служат, как правило, теоремы существования и единственности, которые получаются в результате тшательных математических исследований задач гидродинамики. Основные (локальные) теоремы сушествования для уравнений Навье-Стокса (2.4a)-(2.4d) утверждают, что задача Коши $[(2.4 \mathrm{a})-(2.4 \mathrm{~d}),(3.1)]$ и начально-краевая задача $[(2.4 \mathrm{a})-(2.4 \mathrm{~d})$, $(3.1),(3.2),(3.6)]$ разрешимы локально по времени, т.е. они имеют решение на некотором временном интервале $\left(0, t^{*}\right)$, зависящем от начальных данных. Вопрос о разрешимости этих задач на произвольном временном интервале $\left(0, t^{* *}\right)$, не зависяшем от начальных данных (глобальное существование), остается открытьм (но некоторые новые результаты получены недавно Лионсом [20]). 
Отметим, что для одномерных вязких сжимаемых течений в [21] установлены глобальные теоремы сушествования, т.е. результаты о разрешимости на произвольном временном интервале.

Некоторые результаты в случае сжимаемой жидкости. В статье Валли [18] можно найти разные математические результаты, касающиеся существования единственного “гладкого" решения стационарных и нестационарных задач для баротропной жидкости в ограниченной области (слабые решения не рассматриваются). Автор изучает сначала сжмимаемую невязкую жидкость $(p=P(\rho) \equiv \rho, \mu=0$, $\left.\mu_{v}=0\right)$, для которой задача записывается в виде

$$
\begin{gathered}
\left.\frac{\partial \boldsymbol{u}}{\partial t}+(\boldsymbol{u} \cdot \nabla) \boldsymbol{u}+\nabla \eta=0 \quad \text { в } \quad Q_{T}:=\right] 0, T[\times \Omega, \\
\frac{\partial \eta}{\partial t}+\boldsymbol{u} \cdot \nabla \eta+\operatorname{div} \boldsymbol{u}=0 \quad \text { в } Q_{T} \\
\left.\left.(\boldsymbol{u} \cdot \boldsymbol{n})\right|_{\partial \Omega}=0 \text { на } \Sigma_{T}:=\right] 0, T[\times \partial \Omega \\
\left.\boldsymbol{u}\right|_{t=0}=\boldsymbol{u}^{0}(\boldsymbol{x}),\left.\quad \eta\right|_{t=0}=\eta^{0}(\boldsymbol{x}) \text { в } \Omega .
\end{gathered}
$$

Эти уравнения образуют симметрическую гиперболическую (эйлерову) систему первого порядка, в которой

$$
\eta(t, \boldsymbol{x}):=\log \rho .
$$

Идея доказательства сушествования решения состоит в преобразовании задачи (5.1)-(5.4) в эквивалентную систему для вихря $(\omega=\nabla \wedge \boldsymbol{u})$ и дивергенции вектора $\boldsymbol{u}$. Затем для доказательства существования применяется принцип неподвижной точ$\kappa u$ типа Шаудера. Мы не приводим подробности доказательства, отсылая читателя к статьям Бейрао да Вейга [22], [23].

Единственность решения была установлена в работах Граффи [24] и Серрина [25]. Сушествование решения задачи Коши (т.е. в случае $\Omega=\mathbb{R}^{3}$ ) является следствием общей теории квазилинейных симметрических гиперболических систем (см., например, Като [26]). В заключение отметим, что контрпример к глобальному существованию гладкого решения задачи Коши построен в работе Сидериса [27].

В случае сжимаемой обтемно-вязкой жидкости $\left(p=P(\rho), \mu=0, \mu_{v}>0\right)$ отметим только интересньй результат Сеччи [28], который при некоторых предположениях можно обобщить на случай полной системы (2.4b) (с $\mu=0),(2.4 \mathrm{a})$ и (2.4c) $($ c $k>0$ или $k<0)$. Необходимо отметить, что уравнение $(2.4 \mathrm{~b})(\mathrm{c} \mu=0)$ имеет второй порядок, но не является параболическим в обычном смысле. С другой стороны, функция div $\boldsymbol{u}$, по сушеству, удовлетворяет уравнению теплопроводности с условием Неймана на гранище, и это является решаюшим замечанием для построения решения. Вопрос о сушествовании глобального решения остается открытылм (даже при условии малости начальных данных) в случае, когда размерность пространства больше единицы. (В одномерном случае нет разнииы между вязкой и объемно-вязкой жидкостями.) По этому поводу с математической точки зрения можно сказать, что если пространственная размерность $d$ больие единищы, то облемно-вязкая жсидкость больше похожа на невязкую, чем на вязкую жидкость.

В случае сжимаемой вязкой жидкости $\left(p=P(\rho), \mu>0, \mu_{v} \geqslant 0\right)$ справедлива теорема о глобальном по времени сушествовании решения, установленная в статье Валли [29], который развил некоторые идеи работ Мацумура [30] и Мацумура, 
Нишида [31], [32]. Этот результат Валли был перенесен на полную систему уравнений Навье-Стокса (2.4a)-(2.4c) в работе Валли и Заяцковского [33], где можно найти также некоторые результаты, касаюшиеся задачи втекания-вытекания (т.е. в случае $\left.\left.(\boldsymbol{u} \cdot \boldsymbol{n})\right|_{\partial \Omega} \neq 0\right)$.

В баротропном случае мы для простоты предположим, что

$$
\mu=1, \quad \mu_{v} \geqslant \frac{2}{d}, \quad \rho^{0}(x)=\rho^{*}=1, \quad P(\rho)=\rho,
$$

и рассмотрим новую неизвестную функцию

$$
\sigma(t, \boldsymbol{x})=\rho(t, \boldsymbol{x})-1, \text { которая удовлетворяет условию } \int_{\Omega} \sigma d \Omega=0 \text {. }
$$

При этом исходную систему уравнений можно переписать в виде

$$
\begin{gathered}
\left.\frac{\partial \boldsymbol{u}}{\partial t}-\Delta \boldsymbol{u}-\nabla \operatorname{div} \boldsymbol{u}+\nabla \sigma=\boldsymbol{f}+\boldsymbol{H} \quad \text { в } \quad Q_{\infty}:=\right] 0,+\infty[\times \Omega, \\
\frac{\partial \sigma}{\partial t}+\operatorname{div} \boldsymbol{u}=L \quad \text { в } \quad Q_{\infty}, \\
\boldsymbol{H}:=-(\boldsymbol{u} \cdot \nabla) \boldsymbol{u}-\frac{\sigma}{1+\sigma}[-\nabla \sigma+\Delta \boldsymbol{u}+\nabla \operatorname{div} \boldsymbol{u}], \\
L:=-\sigma \operatorname{div} \boldsymbol{u}-\boldsymbol{u} \cdot \nabla \sigma .
\end{gathered}
$$

Вклад слагаемых $\nabla \sigma$ и $\operatorname{div} \boldsymbol{u}$ можно исключить, умножая уравнения (5.5) и (5.6) на $\boldsymbol{u}$ и $\sigma$ соответственно и интегрируя по $\Omega$. В самом деле, складывая эти два уравнения и учитывая соотношение

$$
\int_{\Omega} \sigma \operatorname{div} \boldsymbol{u} d \Omega=-\int_{\Omega} \nabla \sigma \cdot \boldsymbol{u} d \Omega
$$

получим (через $\|\cdot\|_{k}$ обозначается любая эквивалентная норма в $H^{k}(\Omega)$ ):

$$
\frac{d}{d t}\left[\|\boldsymbol{u}\|_{0}^{2}+\|\sigma\|_{0}^{2}\right]+\|\nabla \boldsymbol{u}\|_{0}^{2} \leqslant c\|\boldsymbol{f}\|_{-1}^{2}+N L,
$$

где через $N L$ обозначается сумма норм некоторых нелинейных слагаемых (которые вследствие предположения о малости данных задачи являются "хорошими" в том смысле, что оцениваются через линейные члены). Эту процедуру можно повторить для $\partial \boldsymbol{u} / \partial t$ (а также для "касательных" и "внутренних" производных функции $\boldsymbol{u}$ до второго порядка), так как во всех случаях граничные условия допускают такое же интегрирование по частям, как и в (5.9). Читатель может найти подробности вывода "хороших" оценок для нелинейных членов в упомянутой выше статье Валли. Однако случай небаротропного сжимаемого течения требует некоторых дополнительных оценок, которые позволяют "улучшать" энергию и диссипативные члены. 
Наконец, в недавней статье Заящковского [34] рассматривается движение вязкой сжимаемой жидкости в $\mathbb{R}^{3}$, которая ограничена свободной поверхностью, находящейся под поверхностным натяжсением и постоянным внешним давлением. В предположении, что начальная плотность достаточно близка к постоянной, начальная область достаточно близка к шару, начальная скорость достаточно мала, а внешшяя сила отсутствует, установлено глобальное по времени существование решения, которое в любой момент времени удовлетворяет свойствам, предписанным в начальном положении.

В заключение еще раз упомянем обзорную статью Солонникова и Кажихова [21], где можно найти теорию существования для вязкой жидкости, развитую к началу 80-х годов, и подробную библиографию.

Отметим также, что теорема единственности была, в частности, доказана Валли [35] и Итая [36]. В работе Валли [18] можно найти подробную библиографию, касаюшуюся математических результатов для сжимаемых течений.

Случай несжимаемой жидкости. Книга Шинброта [12] содержит полную и детальную математическую теорию (развитую к началу 70-х годов) для несжимаемых вязких уравнений Навье ( слабые и сильные решения, существование и единственность слабого решения, сушествование и единственность сильного решения, $р е$ продуктивное свойство для уравнений Навье). В частности, если внешняя сила в уравнении Навье имеет период $\tau=t_{1}-t_{0}$, где $t_{1}$ и $t_{0}$ - любые два момента времени $t$, то из репродуктивного свойства очевидным образом следует периодичность решения. Точное математическое определение классического, слабого и сильного решений уравнений Навье можно найти в книгах Шинброта [12; с. 155, 193] и Темама [13; раздел 3.6]. В книге Темама [13] содержатся более новые результаты о стационарных и нестационарных уравнениях Навье, а также о так называемых стационарных уравнениях Стокса:

$$
-\nu \Delta \boldsymbol{u}+\nabla p=\boldsymbol{f}, \quad \operatorname{div} \boldsymbol{u}=0 \quad \text { в } \Omega
$$

с граничным условием

$$
\boldsymbol{u}=0 \text { на } \Gamma=\partial \Omega \text {. }
$$

Необходимо отметить, что существование слабого решения следует из некоторых неравенств. Такие неравенства, которые справедливы, если решение существует, и из которых вытекает его сушествование, называются априорными оценками. Сильное решение также удовлетворяет некоторой априорной оценке и в этом смысле не отличается от слабого решения. Отметим, что вместо априорных оценок можно использовать метод Галеркина, и это дает новое доказательство сушествования слабого решения начальной задачи для уравнений Навье (см., например, Темам [13; гл. II, $\S 1]$ ).

В статье Хейвуда [37] построены классические решения уравнений Навье как для стационарных, так и для нестационарных граничных задач в произвольной трехмерной области с гладкой границей. При доказательстве основной теоремы существования в случае нестационарных задач предполагается лишь, что начальная скорость обладает конечным интегралом Дирихле. Решение строится методом Галеркина, причем в качестве базисных функций берутся собственные функции оператора Стокса. 
Имеется обширная литература, касаюшаяся регулярности решений уравнений Стокса (см. библиограффию в книге Хейвуда [37]). Что касается убвьвания при $t \rightarrow \infty$ решений во внешней области, Хейвуд показал, что в случае неоднородныъх задач, управляюших возмушениями, которые изучаются в теории устойчивости решений внешних стационарных задач, имеет место убывание порядка $t^{-1 / 4}$. В случае однородных граничных условий и внешних сил для нестационарных задач установлено убьвание порядка $t^{-1 / 2}$.

Наконец, рассматривая задачу для уравнений Навье в неограниченных областях общего вида, Хейвудотмечает необходимость наложения "вспомогательных условий", отвечающих данной области.

В недавней статье Саймона [38] рассматривается течение жидкости, являющейся вязкой, несжимаемой и неоднородной, т.е. с переменной плотностью (вязкая изопикническая модель). Течение рассматривается в области $\Omega \subset \mathbb{R}^{3}$ с границей $\Gamma$ на временном интервале $[0, T]$. Скорость $\boldsymbol{u}$, давление $p$ и плотность $\rho$ удовлетворяют уравнениям

$$
\begin{gathered}
\frac{\partial(\rho \boldsymbol{u})}{\partial t}+\nabla \cdot(\boldsymbol{u} \rho \boldsymbol{u})-\mu \Delta \boldsymbol{u}=\rho \boldsymbol{f}-\nabla p, \\
\frac{\partial(\rho \boldsymbol{u})}{\partial t}+\nabla \cdot(\rho \boldsymbol{u})=0, \\
\nabla \cdot \boldsymbol{u}=0
\end{gathered}
$$

в цилиндре $\Omega \times] 0, T[$, дополненным следуюшими граничными и начальными условиями:

$$
\begin{gathered}
\boldsymbol{u}=0 \text { на } \Gamma \times] 0, T[, \\
\rho=\rho^{0}, \quad \rho \boldsymbol{u}=\rho^{0} \boldsymbol{u}^{0} \text { в } \Omega \text { при } t=0 .
\end{gathered}
$$

Автор устанавливает существование глобального по времени решения $(\boldsymbol{u}, p, \rho)$, для которого $\rho \boldsymbol{u}$ удовлетворяет слабым начальньм условиям. Для этого решения функции $\boldsymbol{u}$ и $\rho \boldsymbol{u}$ не обязаны быть непрерывными по $t$, и поэтому значения $\boldsymbol{u}(t=0)$ и $(\rho \boldsymbol{u})(t=0)$, вообще говоря, не определены.

При этом не требуется, чтобы начальная плотность $\rho^{0}$ имела положительную нижнюю грань. Если к тому же $\rho^{0}$ не слишком мала, но, возможно, обрашается в нуль в некоторых точках, $\boldsymbol{u}$ непрерывна по $t$ при $t=0$ и удовлетворяет сильным начальным условиям $(\rho \boldsymbol{u})(t=0)=\rho^{0} \boldsymbol{u}^{0}$ и $\boldsymbol{u}(t=0)=\boldsymbol{u}^{0}$, то $(\boldsymbol{u}, p, \rho)$ - глобальное сильное решение.

Касаясь сушествования установившегося течения Навье вне некоторого тела на плоскости, отметим работу Амика [39]. В этой работе рассматривается проблема нахож дения решения $(p, \boldsymbol{w})$ стационарных уравнений Навье во внешней плоской области $P$ :

$$
\begin{gathered}
-\nu \Delta \boldsymbol{w}+(\boldsymbol{w} \cdot \nabla) \boldsymbol{w}=-\nabla p, \quad \nabla \cdot \boldsymbol{w}=0 \text { в } \quad P, \\
\boldsymbol{w}=0 \text { на } \Gamma, \quad \boldsymbol{w} \rightarrow \boldsymbol{w}_{\infty} \quad \text { при }|\boldsymbol{x}| \rightarrow \infty .
\end{gathered}
$$

В 1933 г. Ж. Лере [40] изучил задачу (5.17), (5.18), решая последовательность приближенных задач в кольцеобразной ограниченной области $P_{R}$ (пересечение $P$ и шара 
$\left.|\boldsymbol{x}|^{2}<R^{2}\right)$, и установил существование решения $\left(p_{R}, \boldsymbol{w}_{R}\right)$, удовлетворяющего дополнительному условию

$$
\int_{P_{R}}\left|\nabla \boldsymbol{w}_{R}\right|^{2} d \Omega_{R}<\mathrm{const}
$$

В книге [41] содержатся различные математические результаты, касаюшиеся уравнений Навье и Эйлера (по поводу уравнений Навье-Стокса для сжимаемой жидкости см. статью Валли [42]). Этим вопросам посвящена также книга [43].

В заключение приведем некоторые результаты, касаюшиеся решений (несжимаемых) уравнений Эйлера.

Сушествование гладкого решения для уравнений Эйлера было установлено многими авторами (см., например, Темам [44]). В случае глобально аналитических данных (на компактном многообразии или в ограниченной области) существование доказано в работе Бауэнди, Гулауика [45]. Метивье [46] установил локальное сушествование для аналитических псевдодифференциальных операторов и аналитических данных. Априорные результаты о гладкости для уравнений Эйлера в случае глобально аналитических данных доказаны в работах Бардоса [47], Бардоса, Бенашура, Цернеpa [48] и Бенашура [49]. По поводу распространения локальной аналитичности для (несжимаемых) уравнений Эйлера см. работу Алинака и Метивье [50]. Обобщения на неоднородные жидкости и граничные задачи получены Ле Белом [51].

Теорема Коши-Ковалевской. Прежде всего отметим, что если $\boldsymbol{U}(t, x, y)$ - неизвестный вектор (с компонентами $\left.U_{i}, i=1, \ldots, l\right)$, а линейная система (для $\boldsymbol{U}$ ) сводится к системе первого порядка относительно $t, x$ и $y$, то ее можно записать в виде

$$
A \frac{\partial \boldsymbol{U}}{\partial t}+B \frac{\partial \boldsymbol{U}}{\partial x}+C \frac{\partial \boldsymbol{U}}{\partial y}+D \boldsymbol{U}=0
$$

где матришы $A, B, C$ и $D$ - гладкие функции от $t, x$ и $y$.

Пусть $S$ - гладкая поверхность, заданная параметрически:

$$
t=t(\alpha, \beta), \quad x=x(\alpha, \beta), \quad y=y(\alpha, \beta),
$$

где $\alpha, \beta$-параметры. Рассмотрим начальное условие, задающее значение $\boldsymbol{U}$ на $S$, т.е.

$$
\boldsymbol{U}[t(\alpha, \beta), x(\alpha, \beta), y(\alpha, \beta)]=\text { заданная гладкая функция переменных } \alpha \text { и } \beta \text {. }
$$

Поверхность $S$ называется характеристической, если она ориентирована так, что дифференциальные уравнения налагают некоторые ограничения на начальную функцию (5.21), заданную на поверхности $S$.

Поэтому мы ищем такую линейную комбинацию уравнений системы (5.20), что все неизвестные функции $U_{i}$ (компоненты $\boldsymbol{U}$ ) дифференшируются по направлениям, лежашим в некоторой плоскости. Если поверхность $S$ касается этой плоскостив точке $P$, то в этой точке упомянутая вьше линейная комбинация выражается в терминах производных по $\alpha$ и $\beta$, и поэтому полученное дифференциальное уравнение (линейная комбинация) налагает ограничения на начальную функцию (5.21) в точке $P$. В этом случае 
плоскость назьвается характеристической плоскостью в точке $P$, а поверхность $S$ назьвается характеристической в точке $P$. Если $S$ является характеристической во всех точках, то она называется характеристической поверхностью системы уравнений (см., например, книгу Рихтмайера [19; т. I, гл. 17]).

Предположим, что рассматриваемая вьше линейная комбинация получена умножением системы (5.20) на вектор $\boldsymbol{W}=\boldsymbol{W}(t, x, y)$ слева. Тогда неизвестная функция $U_{i}$ дифференцируется вдоль вектора (в плоскости $(t, x, y))$ с направляющими косинусами, пропорциональными $\left(\boldsymbol{W}^{T} A\right)_{i},\left(\boldsymbol{W}^{T} B\right)_{i},\left(\boldsymbol{W}^{T} C\right)_{i}$. Следовательно, если $\lambda, \mu, \nu$ - направляющие косинусы нормали к $S$ в точке $P$, то условие характеристичности поверхности $S$ имеет вид

$$
\lambda \boldsymbol{W}^{T} A+\mu \boldsymbol{W}^{T} B+\nu \boldsymbol{W}^{T} C=0 .
$$

Это означает, что $\boldsymbol{W}$ - левый собственньй вектор матрицы

$$
\Lambda=\lambda A+\mu B+\nu C,
$$

соответствующий нулевому собственному значению, необходимым и достаточным условием сушествования которого является равенство

$$
\operatorname{det} \Lambda=0 \text {. }
$$

Три неизвестные функции $\lambda, \mu, \nu$ должны удовлетворять также уравнению

$$
\lambda^{2}+\mu^{2}+\nu^{2}=1 \text {. }
$$

Таким образом, мы получили два уравнения, и поэтому можно ожидать, что существует одно или несколько однопараметрических семейств решений. Если эти решения являются вешественнозначными, то существуют соответствуюшие однопараметрические семейства характеристических плоскостей.

В случае двумерных невязких гидродинамических уравнений Эйлера (характеристическая форма (4.27) этих уравнений приведена в четвертом параграфе) имеются два таких семейства, одно из которых состоит из всех плоскостей, касательных к траектории частипы в пространстве $(t, x, y)$ (в уравнении $(4.27 \mathrm{~b})$ направления дифференцирования составляют плоскость, которая касается траектории частицы и параллельна $\mu$ ), а другое - из всех плоскостей, касательных звуковому конусу (Маха) (в уравнении $(4.27 \mathrm{c})$ направления дифференщирования составляют плоскость, которая касается звукового конуса и такова, что ее пересечение с плоскостью $(x, y)$ перпендикулярно $\lambda$ ).

Отметим, что если $M=|\boldsymbol{u}| / c$ - локальное число Маха, то угол $\mu$, для которого

$$
\sin \mu=\frac{1}{M}
$$

назьвается углом Маха; он является действительным, только когда $M \geqslant 1$, и не сушествует для дозвуковых течений $(M<1)$. Для сверхзвуковых $(M>1)$ течений возмушения сосредоточены в правом круговом конусе, а $\mu$-полукруговой вертикальный угол этого конуса - так назьваемьй звуковой конус (см., например, книгу Варда $[52 ;$ гл. 3$])$. 
Гидродинамическая задача является, конечно, нелинейной, и поэтому поверхность (плоскость) $t=$ const не может быть характеристической, так как если бы одна из характеристических плоскостей имела вид $t=$ const, то скорость распространения сигнала была бы бесконечной, тогда как в гидродинамике скорости жидкости и звука конечны при любом выборе начальных данных, а максимальная скорость распространения сигнала совпадает с суммой этих двух скоростей. Этот факт следует также из того, что в гидродинамике матрища $A$ в уравнении (5.20) является единичной. Предположим теперь, что коэффициенты матриц $A, B, C$ и $D$ в уравнении (5.20) зависят от компонент вектора $\boldsymbol{U}$, а также от $t, x$ и $y$, т.е. рассматривается случай нелинейной гидродинамики. Тогда уравнения (5.20) являются квазилинейными. При этом определения и выводы - такие же, как и для линейных уравнений, однако точки зрения несколько разные: для заданной системы уравнений поверхность $S$ может быть или не быть характеристической в зависимости от начальных функций, заданных на $S$, т.е. компонент векторного поля $(5.21)$ на $S$, так как $A, B$ и $C$ зависят от $\boldsymbol{U}$. По этой причине часто говорят о характеристичности или нехарактеристичности начальных функций по отношению к заданной поверхности $S$.

Задача Коши (задача определения функции $\boldsymbol{U}(t, x, y)$ по данным Кошш $(5.21)$ и дифференциальному уравнению (5.20)) назьвается аналитической, если поверхность $S$ и все заданные функции являются аналитическими. Для аналитичности $S$ достаточно, чтобы функции $t(\alpha, \beta), x(\alpha, \beta)$ и $y(\alpha, \beta)$ были аналитическими, а ранг матрицы

$$
\left(\begin{array}{lll}
\frac{\partial x}{\partial \alpha} & \frac{\partial y}{\partial \alpha} & \frac{\partial t}{\partial \alpha} \\
\frac{\partial x}{\partial \beta} & \frac{\partial y}{\partial \beta} & \frac{\partial t}{\partial \beta}
\end{array}\right)
$$

равнялся двум всюду на поверхности $S$.

Теперь мы сформулируем без доказательства один из вариантов теоремь КошиКовалевской в случае трех независимых переменных $t, x, y$.

Предположим, что даннье Коши (5.21) аналитичны на аналитической поверхности $S$ и нехарактеристичны по отношению $\kappa S$ в некоторой (двумерной) окрестности точки $P$ на $S$. Пусть матрицы $A, B, C$ и $D$ из уравнения (5.20) аналитичны как функции от $t, x, y$ и компонент вектора $\boldsymbol{U}$. Тогда найдется трехмерная окрестность точки $P$, в которой задача Коши имеет единственное решение.

В наиболее распространенном случае поверхность $S$ совпадает с плоскостью $(x, y)$ (т.е. $t=0)$, и условия теоремы выполнены во всех точках плоскости. При этом если $K$ - компактная область на плоскости, то найдется такой интервал $(-T, T)$, что задача имеет единственное решение для всех $(x, y)$ и $t$, принадлежаших $K$ и $(-T, T)$ соответственно.

В случае невязкой гидродинамической (гиперболической) задачи требование аналитичности можно заменить гладкостью (непрерывной дифференцируемостью).

Ударные волны и другие важные особенности не покрываются теорией Коши-Ковалевской. Более того, если имеется контактньй разрыв или поверхность скольжения, то из-за неустойчивости Тейлора-Гельмгольца может случиться так, что для сушествования решения поверхность и течение по обе ее стороны должны быть не просто гладкими, а аналитическими или, по крайней мере, кусочно-аналитическими. 
Если существование и единственность решения не доказаны, то удобно принимать некоторую рабочую гипотезу или налагать дополнительное условие, чтобы создать базу для дальнейших исследований. Разумным условием в случае начальной задачи для невязкой (әйлеровой) гидродинамики является следуюшее предположение (уравнение состояния также предполагается кусочно-аналитическим):

если начальнье даннье - кусочно-аналитические, то, по крайней мере, на конечном интервале времени существует кусочно-аналитическое решение.

Наконец, чтобы дать физическую интерпретацию условия разрешимости относительно производных по времени, рассмотрим линейный случай с двумя независимьми переменными.

В этом случае уравнения (5.20) записьваются в виде

$$
A \frac{\partial \boldsymbol{U}}{\partial t}+B \frac{\partial \boldsymbol{U}}{\partial x}+C \boldsymbol{U}=0
$$

где $A, B$ и $C$-матришы размера $l \times l$, элементами которых являются гладкие функции от $t$ и $x$. Снова рассмотрим задачу Коши (начальную задачу), которая состоит из уравнения (5.25) и начального условия, согласно которому

$$
\boldsymbol{U}(0, x) \text { задана при всех } x \text {. }
$$

Если определитель $\operatorname{det} A$ отличен от нуля в некоторой области $R$ (плоскости $(t, x))$, содержащей ось $x$ (или, более общо, часть оси $x$ ), то сужение функции $\partial \boldsymbol{U} / \partial t$ на ось $x$ в области $R$ можно выразить из уравнения (5.25) в терминах заданной функции $\boldsymbol{U}(0, x)$.

Дифференцируя уравнение (5.25) по $t$, мы можем выразить $\partial^{2} \boldsymbol{U} / \partial t^{2}$ через (уже найденные) сужения функций $\boldsymbol{U}$ и $\partial \boldsymbol{U} / \partial t$ на ось $x$, и т. д. Поэтому если $\boldsymbol{U}(0, x)$ бесконечно дифференцируема по $x$, то все производные $\partial^{k} \boldsymbol{U} / \partial t^{k}$ можно найти, что позволяет построить степенной ряд

$$
\sum_{k=0}^{\infty} \frac{t^{k}}{k !}\left(\frac{\partial^{k} \boldsymbol{U}}{\partial t^{k}}\right)_{t=0}
$$

Можно показать (это частный случай теоремы Коши-Ковалевской), что если начальная функция $\boldsymbol{U}(0, x)$ и матришы $A, B, C$ аналитичны по $t, x$, то ряд (5.26) сходится, когда $t$ принадлежит некоторому интервалу $(-T, T)$, где $T$ может зависеть от $x$. При этом полученные функции переменных $t$ и $x$ удовлетворяют уравнению (5.25).

Таким образом,

решение начальной задачи, рассматриваемой в области $R$, построено в некоторой окрестности оси $x$.

В случае, когда $\operatorname{det} A$ обращается в нуль на оси $x$ в области $R$, получается совершенно другой результат. Обозначим через $\boldsymbol{V}=\boldsymbol{V}(x)$ левый собственный вектор 
матрищы $A$, соответствуюший нулевому собственному значению. Умножая уравнение $(5.25)$ на $\boldsymbol{V}^{T}$ слева, легко понять, что начальная функция $\boldsymbol{U}(0, x)$ должна удовлетворять условию

$$
\left[\boldsymbol{V}^{T} B \frac{\partial}{\partial x}+\boldsymbol{V}^{T} C\right] \boldsymbol{U}(0, x)=0
$$

для любого левого собственного вектора $\boldsymbol{V}$; в противном случае начальная задача не имеет решения! Более того, даже если начальная функция удовлетворяет условию (5.27), описанный вьше метод построения решения с помошю степенных рядов, вообще говоря, неприменим, так как из уравнения (5.25) сужения производных $\partial \boldsymbol{U} / \partial t$, $\partial^{2} \boldsymbol{U} / \partial t^{2}, \ldots$ на ось $x$ определяются не однозначно. Например, к любому значению $\partial \boldsymbol{U} / \partial t$, найденному из уравнения (5.25), можно добавить произвольньй вектор, который пропорционален правому собственному вектору матрицы $A$, соответствуюшему нулевому собственному значению. Резюмируя вышесказанное, можно утверждать, что $[19 ;$ т. I, с. 439]

начальная задача имеет единственное локальное (по времени) решение, если $\operatorname{det} A \neq 0$ в $R ;$ в то же время, если $\operatorname{det} A=0$ в $R$, то решение, вообще говоря, не существует и не единственно (если оно существует).

\section{§ 6. Вихревые пелены и ударные слои}

Течение (невязкой непроводящей) жидкости не обязано быть непрерьвным, как предполагалось выше, и на поверхности, на которой скорость терпит разрыв, дифференциальные уравнения движения Эйлера не выполняются. Поэтому требуются новые уравнения, связьвающие переменные на разных сторонах таких поверхностей.

Пусть $G$ - некоторая фиксированная поверхность, находящаяся в стационарном әйлеровом течении. При условии, что течение проходит через поверхность $G$, индексами + и - будем обозначать значения переменных на передней ${ }^{1}$ и задней сторонах $G$ соответственно. Пусть $\boldsymbol{n}$ - единичная нормаль к $G$.

Тогда закон сохранения массы на $G$ приводит к уравнению

$$
\rho_{+} \boldsymbol{u}_{+} \cdot \boldsymbol{n}=\rho_{-} \boldsymbol{u}_{-} \cdot \boldsymbol{n}
$$

а из закона сохранения линейного импульса следует, что

$$
p_{+} \boldsymbol{n}+\rho_{+} \boldsymbol{u}_{+} \boldsymbol{u}_{+} \cdot \boldsymbol{n}=p_{-} \boldsymbol{n}+\rho_{-} \boldsymbol{u}_{-} \boldsymbol{u}_{-} \cdot \boldsymbol{n} .
$$

Умножая уравнение (6.2) векторно на $\boldsymbol{n}$ и используя (6.1), получим

$$
\rho_{+} \boldsymbol{u}_{+} \cdot \boldsymbol{n}\left(\boldsymbol{u}_{+}-\boldsymbol{u}_{-}\right) \wedge \boldsymbol{n}=0
$$

что является условием на скорости, которое необходимо для существования разрыва в стационарном невязком течении.

\footnotetext{
${ }^{1}$ Передней называется та сторона $G$, на которую натекает поток. Прим. перев.
} 
Полученное вьше условие (6.3) показьвает, что имеются две нетривиальнье возможности.

В первом случае имеем

$$
\boldsymbol{u}_{+} \cdot \boldsymbol{n}=\boldsymbol{u}_{-} \cdot \boldsymbol{n}=0 \text { и } \quad\left(\boldsymbol{u}_{+}-\boldsymbol{u}_{-}\right) \wedge \boldsymbol{n} \neq 0 .
$$

При этом из (6.2) также следует, что

$$
p_{+}=p_{-}
$$

Таким образом, течение через поверхность отсутствует, и давление непрерывно на поверхности; в этом случае поверхность является контактным разрывом или вихревой пеленой, что эквивалентно поверхностному распределению завихренности. Разрывы такого сорта могут возникать в течениях всех типов; при этом стационарные уравнения движения на линиях обтекания не нарушаются, так как они не пересекают поверхность разрьва.

Во втором случае имеют место соотношения

$$
\left(\boldsymbol{u}_{+}-\boldsymbol{u}_{-}\right) \wedge \boldsymbol{n}=0 \text { и }\left(\boldsymbol{u}_{+}-\boldsymbol{u}_{-}\right) \cdot \boldsymbol{n} \neq 0
$$

в силу которых касательная компонента скорости непрерывна, и имеется разрывный поток через поверхность; из уравнений (6.1) и (6.2) следует, что плотность и давление такжсе терпят разрыв. Такие разрывы называются скачками уплотнения или ударными волнами.

В силу закона сохранения әнергии в любой точке поверхности полная әнергия на единицу массьи

$$
E=e+\frac{p}{\rho}+\frac{1}{2} \boldsymbol{u}^{2}
$$

одинакова на обеих ее сторонах. Это означает, что для стационарного (вихревого) течения уравнение Бернулли имеет вид

$$
e+\frac{p}{\rho}+\frac{1}{2} u^{2}=h^{*}
$$

где $h^{*}$ - значение удельной энтальпии $h$ через поверхность в застойной зоне (при $\boldsymbol{u}=0)$. Для идеального газа с постоянными теплоемкостями (в этом случае $h=[\gamma /(\gamma-1)](p / \rho))$ имеем

$$
\frac{\gamma p_{+}}{(\gamma-1) \rho_{+}}+\frac{1}{2}\left(\boldsymbol{u}_{+}\right)^{2}=\frac{\gamma p_{-}}{(\gamma-1) \rho_{-}}+\frac{1}{2}\left(\boldsymbol{u}_{-}\right)^{2} .
$$

Согласно этому условию скачка разрьв на ударной волне может быть либо сжимающим, либо разрежсающим. Можно, однако, показать, что энтропия $S$ терпит разрьв на ударной волне, причем она возрастает в направлении, в котором скорость убывает, а давление и плотность растут, и наоборот.

Таким образом, из второго закона термодинамики получаем следуюший вывод:

ударные волны физически могут существовать, только если разрыв является сжимающим; при әтом течение впереди ударной волны должсно быть сверхзвуковым. 
Вследствие изменения энтропии поток, гомэнтропический (в этом случае удельная энтропия $S$ одинакова для всех частиц жидкости) до прохождения через ударную волну, не обязан быть таковым после ударной волны, а безвихревый $(\omega=\nabla \wedge \boldsymbol{u}=0)$ поток, вообще говоря, не остается таковым после прохождения через ударную волну. Достаточно полный обзор свойств ударных волн можно найти в книге Куранта и Фридрихса [53], см. также [54; гл. IX].

Условия на скачке, связанные с законом сохранения. В обшем случае (см., например, обзорную статью Жермэна [55]) закон сохранения утверждает, что для заданного вешества (жидкости) в области $\Omega$, ограниченной замкнутой поверхностью $\partial \Omega$, за которой идет наблюдение в процессе движения, объем “поступлений” извне (соответствуюшая объемная плотность определяется компонентами $F_{i j}$ ) равен сумме "исходящего потока" через $\partial \Omega$ (его поверхностная плотность задается набором $a_{i j k}$ ) и того, что “остается" внутри $\Omega$ (объемная плотность задается матрицей $A_{i j}$ ). В этой формулировке мы используем ортогональные декартовы координаты и предполагаем, что величины $F_{i j}$ и $A_{i j}$ (для которых ниже вьписывается соотношение (6.8)) являются компонентами тензора второго порядка, тогда как $a_{i j k}$ - компоненты тензора третьего порядка. Точная формулировка закона сохранения имеет вид

$$
\frac{D}{D t} \iiint_{\Omega} A_{i j} d v+\iint_{\partial \Omega} a_{i j k} n_{k} d s=\iiint_{\Omega} F_{i j} d v
$$

где вектор $\boldsymbol{n}$ (с декартовыми координатами $n_{k}$ ) - внешняя единичная нормаль, а $D / D t$ - оператор полной производной.

Предположим теперь, что заданная область $\Omega$ представляется в виде объединения двух подобластей $\Omega_{+}$и $\Omega_{-}$, примыкающих к общей границе $G$, которая содержится в $\Omega$ и касательная плоскость к которой непрерьвно зависит от точки касания $P$. Обозначим через $\boldsymbol{W}(t, \boldsymbol{x})$ поле скоростей точек поверхности $G$, а через $\boldsymbol{N}$ - единичную нормаль к $G$, приложенную к точке $P$ и направленную внутрь области $\Omega_{-}$. Пусть вектор $\boldsymbol{U}(t, \boldsymbol{x})$ с декартовыми координатами $U_{i}$ обозначает скорость частицы, расположенной в точке $P$ в момент времени $t$; тогда $\boldsymbol{V}(t, \boldsymbol{x})=\boldsymbol{U}-\boldsymbol{W}$ - скорость среды относительно поверхности $G$. Наконец, обозначим через $[f]$ скачок функции $f$ при пересечении границы $G$ в направлении $\boldsymbol{N}$.

Теперь мы можем сформулировать следуюшую теорему [55; с. 147].

Пусть функиии $A_{i j}, a_{i j k}, F_{i j}$ и $U_{i}$, определенные в области $V$ и имеющие кусочно-непрерывные ограниченные производнье, удовлетворяют ассоциированному дифференциальному уравнению с частными производньми

$$
\frac{\partial}{\partial t}\left(A_{i j}\right)+\frac{\partial}{\partial x_{k}}\left(A_{i j} U_{k}\right)+\frac{\partial}{\partial x_{k}}\left(a_{i j k}\right)=F_{i j}
$$

в любой точке непрерывности и соотношению

$$
\left[A_{i j} v+a_{i j k} N_{k}\right]=0
$$

$($ әде $v=\boldsymbol{V} \cdot \boldsymbol{N})$ на любой поверхности разрыва $G$. Тогда для произвольной подобласти $\Omega$ области $V$ имеет место закон сохранения (6.8). 


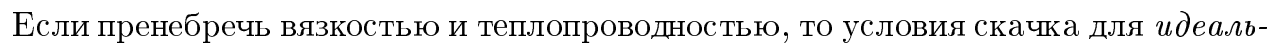
ной жицдости примут вид

$$
\begin{gathered}
{[\rho v]=0, \quad[p \boldsymbol{N}+\rho v \boldsymbol{U}]=0,} \\
{\left[\rho\left(e+\frac{1}{2} \boldsymbol{U}^{2}\right)+p(v+w)\right]=0,}
\end{gathered}
$$

где $w=\boldsymbol{N} \cdot \boldsymbol{W}$.

Таким образом, функция $m=\rho v$ непрерывна в направлении нормали к $G$, и $е с л и$ $m=0$, то $G$ называется контактныцм разрывом; при этом давление $p$ непрерывно на $G$, но плотность $\rho$ и температура $T$ могут иметь разрывы на $G$.

Если $m \neq 0$, то поверхность $G$ назьвается ударной волной, и равенства (6.11) можно записать в виде (соотношения на ударной волне)

$$
\begin{aligned}
{[\rho v] } & =0, & & {\left[p+\rho v^{2}\right]=0, } \\
{\left[\boldsymbol{V}_{T}\right] } & =0, & & {\left[h+\frac{1}{2} v^{2}\right]=0, }
\end{aligned}
$$

где $\boldsymbol{V}_{T}$ - касательная компонента вектора $\boldsymbol{V}$.

Отметим, что соотношения на ударной волне (6.12) можно переписать в терминах только относительной скорости $\boldsymbol{V}$ (ввиду принципа относительности Галилея законы механики имеют одинаковый вид $\theta$ любой инерциальной системе отсчета).

Рассмотрим теперь случай стационарной нормальной ударной волны, отделяющей $\partial в е$ однородные области идеального газа с постоянными удельными теплоемкостями, причем течение в обоих областях предполагается стационарным. Такая ударная волна интерпретируется как плоский разрьв, так что эффектами вязкости и теплопроводности можно пренебречь (образование наклонной ударной волны изучается в книге О'Нейла и Чорлтона [56; раздел 7.3]). Если ударная волна, подобно движению поршня в ударной трубе, является нестационарной, то задачу всегда можно свести к стационарной, предполагая, что вся система движется со скоростью, равной по модулю и противоположной по направлению скорости движушегося ударного фронта.

Давление $p_{1}$, плотность $\rho_{1}$, температура $T_{1}$ и скорость жидкости $u_{1}$ в первой области предполагаются известными, а задача состоит в нахождении этих величин во второй области. В этом случае из соотношений на ударной волне (6.12) мы можем вывести условия Ренкина-Гюгонио:

$$
\begin{aligned}
& \frac{p_{2}}{p_{1}}=\frac{(\gamma-1) \rho_{1}-(\gamma+1) \rho_{2}}{(\gamma-1) \rho_{2}-(\gamma+1) \rho_{1}}, \\
& \frac{\rho_{2}}{\rho_{1}}=\frac{(\gamma-1) p_{1}-(\gamma+1) p_{2}}{(\gamma-1) p_{2}-(\gamma+1) p_{1}} .
\end{aligned}
$$

Предположим теперь, что газ сжимается после прохождения через ударную волну, так что $p_{2}>p_{1}$, и мы можем определить интенсивность ударной волньи по формуле

$$
\pi=\frac{p_{2}}{p_{1}}-1>0 .
$$


При этом удобно ввести критическую скорость звука $c^{*}$, которая достигается при $u=c=c^{*}$; отметим, что $c_{1}^{*}=c_{2}^{*}$.

В этом случае мы можем вьвести следующее соотношение Прандтля:

$$
u_{1} u_{2}=\left(c^{*}\right)^{2}
$$

Введя теперь число Маха $M=u / c$, получим следуюшее важное равенство:

$$
M_{2}^{2}=\frac{1+\frac{1}{2}(\gamma-1) M_{1}^{2}}{\gamma M_{1}^{2}-\frac{1}{2}(\gamma-1)} .
$$

Соотношение (6.16) отражает связь между числами Маха на разных сторонах ударной волны. Часто бывает удобно выразить отношение плотностей $\rho_{2} / \rho_{1}$ в терминах числа Маха $M_{1}$ приходящего потока; в этом случае имеем

$$
\frac{\rho_{2}}{\rho_{1}}=\frac{2+(\gamma-1) M_{1}^{2}}{(\gamma+1) M_{1}^{2}},
$$

и возврашаясь к соотношению Прандтля (6.15), получим (разрежение при прохождении через ударную волну невозможно!) $u_{1}>c^{*}$ и $u_{2}>c^{*}$, откуда вытекает следующее утверждение.

В случае плоской нормальной стационарной ударной волньи приходящий поток является сверхзвуковым $\left(M_{1}>1\right)$, уходящий поток-дозвуковым $\left(M_{2}<1\right)$, а газ при прохожсении через ударную волну сжимается. Ударная волна - необратимый прочесс, после которого әнтропия увеличивается.

Точнее, разность энтропий $S_{2}-S_{1}$ в случае идеального газа имеет такой же знак, что и $M_{1}^{2}-1>0$, и легко понять, что $T_{2}>T_{1}$, откуда получаем следующий вьвод:

переход должен нагревать газ, когда ударная волна превращает сверхзвуковой поток в дозвуковой.

Отметим наконец, что термин "сильная ударная волна" часто используется, когда речь идет о пределе соотношений ударной волны при $M_{1} \rightarrow \infty$; этот предел представляет интерес в связи с задачами сверхзвуковой акустики [57], теорией сильных взрывов и астрофизикой. По поводу теории сверхзвуковых течений см. книгу Гиро [58].

Термин "слабая ударная волна" обозначает предел соотношений на ударной волне при $M_{1} \rightarrow 1$.

Так как увеличение энтропии является мерой неэффективности адиабатических процессов, то утверждение о том, что для слабой ударной волны оно не больше, чем $O\left(\left[M_{1}^{2}-1\right]^{3}\right)$, представляет существенньй практический интерес для сверхзвуковых полетов.

В случае крайне слабых ударных волн скорость, полученная в результате линеаризации (см. книгу Варда [52]), может быть непрерывной, и ударные волны проявляются в том, что производные по пространственньм переменным компонент скорости на соответствующих характеристических поверхностях бесконечны. 
Структура ударного слоя. Ниже предполагается, что все величины, характеризуюшие течение, не зависят от времени и являются функциями только от координаты $x$, а векторы скорости параллельны оси $x$. Согласно теории невязких сжимаемых жидкостей особенностями таких одномерных течений могут быть либо контактные разрывы, либо разрывы типа ударных волн [55].

Легко видеть, что вязкость и теплопроводность в конце концов полностью уничтожают контактный разрыв, так что соответствуюшее стационарное решение представляет собой равномерное течение. С другой стороны, влияние вязкости и теплопроводности на ударную волну проявляется в том, что вместо нее возникает непрерывный переход меж ду начальньм и конечным состояниями. Область непрерьвного перехода назьвается ударныцм слоем; он во многом аналогичен пограничному слою. Отметим, что толшина пограничного слоя имеет порядок $(\mu)^{1 / 2}$ (где $\mu$ - динамическая вязкость), тогда как толшина ударного слоя - порядок $\mu^{*}=\frac{4}{3} \mu$. Обсуждение вопросов, связанных с шириной ударного слоя, можно найти в [59; с. 177], а также в книге Мейера $[2 ;$ с. 170$]$. При нормальных условиях основная часть перехода происходит в очень узкой области, однако, строго говоря, ударньй слой является бесконечно шиироким, и условия впереди и сзади ударной волны следует заменить условиями на бесконечности для приходящего и уходящего потоков соответственно. При этом "условия скачка" должны быть следствием того, учитьваются или нет вязкость и теплопроводность.

В книге Франсуа [60; раздел V.5] методом согласования асимптотических разложений (МСАР) построено асимптотическое решение одномерных уравнений НавьеСтокса при условии, что

$$
M_{+}=\frac{U_{+}}{\left(\gamma R T_{+}\right)^{1 / 2}} \rightarrow 1 \text { и } \operatorname{Re}_{+}=\frac{L U_{+} \rho_{+}}{\mu^{*}} \rightarrow+\infty,
$$

где $L$ - произвольный характерный (макроскопический) масштаб длины. В случае слабой ударной волны, когда $M_{+}$(число Маха приходящего потока) близко к единице, мы можем ввести мальй параметр

$$
\alpha=\left(M_{+}\right)^{2}-1
$$

и заметить, что толщина ударного слоя имеет порядок

$$
\delta=\frac{L}{\alpha \operatorname{Re}_{+}}=\frac{l^{*}}{M_{+}\left[\left(M_{+}\right)^{2}-1\right]},
$$

где $l^{*}=L M_{+} / \operatorname{Re}_{+}-$средний свободный пробег, причем $\delta \gg l^{*}$.

Таким образом, рассматриваемая асимптотическая теория служит адекватной базой для теории слабых ударных волн (заметим, что $\delta / l^{*}$ не зависит от произвольной постоянной $L$ ).

Касаясь приложений МСАР, отметим, что в работе Жермэна [55; раздел IV] приведены асимптотические разложения, полностью описьваюшие (согласно статье Жермэна и Гиро [61]) асимптотическое поведение решения в окрестности ударной волны. Для простоты ограничимся случаем классической газовой динамики. Рассматривается течение вязкой теплопроводящей жидкости и изучается предельное течение, когда эффекты диссипации (характеризуюшиеся малым параметром $\varepsilon$, совпадаюшим с обратным к числу Рейнольдса) стремятся к нулю. 
Первьй вопрос, на которьй следует ответить, состоит в следующем. Предположим, что градиент решения уравнений Навье-Стокса очень велик внутри некоторого "слоя", так что при $\varepsilon \rightarrow 0$ получаем ударную волну на поверхности $G$, где $G$ лежит внутри слоя. Вопрос: как найти правильное асимптотическое разложение такого решения при малых $\varepsilon$ ?

Так как при $\varepsilon \neq 0$ имеем сингулярно возмущенную задачу (невозмущенная задача соответствует $\varepsilon=0)$, трудно ожидать, что асимптотическое разложение будет равномерным всюду (по поводу МСАР см. книгу Лагерстрома [62]).

По этой причине вводятся два типа асимптотических разложений, описывающие поведение решения в окрестности и внутри “слоя". Первое из них, назьваемое внешним разложсением, справедливо вдали от $G$, а именно, на расстоянии порядка некоторой степени $\varepsilon$. Второе разложение, назьваемое внутренним разложением, справедливо внутри слоя.

Таким образом, мы должны построить эти два разложсения, а затем согласовать их так, чтобы они аппроксимировали одно и то же решение уравнений Навье-Стокса.

Описанная выше техника, предложенная в работе Каплуна и Лагерстрома [63], в настоящее время широко используется при асимптотическом моделировании течений жидкости (см., например, Ван Дайк [64], Гиро и Зейтунян [65], Зейтунян [66]).

Отметим, что в работе Жермэна и Гиро [61] получены полные асимптотические разложения с точностью до членов произвольного поря дка. Для построения внешнего разложения фиксируются некоторые геометрические координаты, а затем $\varepsilon$ устремляется к нулю, тогда как внутреннее разложение требует введения новых (криволинейных) координат, связанных с нормалью к $G$, причем расстояние до $G$ по нормали имеет порядок интенсивности диссипации, скажем, $\varepsilon$.

Теперь возникает второй вопрос, связанньй с дальнейшим применением полученных результатов для исследования решений глобальной задачи (на самом деле описание решения в целом зависит от глобальной задачи, тогда как здесь рассматривается только локальная задача). Как отмечено в работе Жермэна [55], если нас интересует только внешнее разложение, то необходимо понять, каким образом разложения на разных сторонах $G$ должны быть связаны друг с другом, чтобы они приближали одно и то же решение. Эти соотношения являются в действительности условиями ударной волны для рассматриваемой задачи. Классические соотношения на скачке приводят к условиям ударной волны для членов до нулевого порядка, и классическая структура ударного слоя также приводит к внутреннему разложению до нулевого порядка.

Стандартный способ изучения таких задач с сингулярными возмущениями состоит в последовательном вычислении членов двух разложений и выписывании соответствующих условий согласования на каждом шаге. Однако более прямой путь использовали Жермэн и Гиро, которые предполагали известньми аналитические свойства разных зависимых переменных в окрестности $G$ и показали, как можно выгислить эти функции, используя уравнения Навье-Стокса. Обоснованность этого метода доказывается проверкой согласованности полученных формул на каждом шаге.

В теории Жермэна, Гиро важны два обстоятельства, а именно, введение функиий класса NS и понятие "связки" таких функций. "Члены связки", фигурируюшие в условиях ударной волны (до первого порядка, см. [55; с. 191 и 192]) важны для состоятельной теории (эти члены учитьвают влияние толщины ударного слоя). Подробности этой теории можно найти в [61]. 
В заключение отметим, что гидродинамика не дает адекватной основы для теории ударных волн, как может показаться из вьшеизложенного! Для достаточно сильных ударных волн (в сверхзвуковых течениях) толщина ударного слоя $\delta$ может быть сравнимой со средним свободньм пробегом $l^{*}$; в этом случае толшина ударного слоя сильно зависит от соотношения между вязкостью и температурой! Естественно, что уравнения Навье-Стокса в этой ситуации являются неадекватны.ми для описания структуры ударного слоя, и необходимо изучать уравнение Больимана кинетической теории газов (по поводу уравнения Больцмана см. статью Серсигнани [67]).

Некоторые свойства вихревой пелены. По определению свободная вихревая пелена представляет собой гладкую поверхность в области, занимаемой жидкостью, при пересечении которой касательная компонента скорости терпит разрьв, тогда как нормальная компонента является непрерьвной. Связь такой поверхности с завихренностью $\omega=\nabla \wedge \boldsymbol{u}$ состоит в следуюшем. Обозначим индексами + и - две стороны вихревой пелены $\Sigma$, а через $\boldsymbol{n}$ - единичную нормаль к $\Sigma$, направленную в сторону с индексом + . Положим

$$
\Omega=n \wedge\left(u_{+}-u_{-}\right)
$$

и назовем величину $\Omega$ поверхностной плотностью завихренности на вихревой пелене $\Sigma$. Векторное поле $\Omega$ касается поверхности $\Sigma$, а так как $\left(\boldsymbol{u}_{+}-\boldsymbol{u}_{-}\right)$также касается $\Sigma$, то правило тройного произведения приводит к соотношению

$$
u_{+}-u_{-}=\Omega \wedge n
$$

Для заданного замкнутого контура $P \subset \Sigma$ обозначим через $s$ и $\tau$ соответственно длину дуги и единичный касательный вектор, согласованный с ориентацией $P$. Тогда $\nu=$ $\boldsymbol{n} \wedge \tau$ и ввиду (6.20) имеет место предельный вариант теоремы Стокса:

$$
\int_{P}\left(\boldsymbol{u}_{+}-\boldsymbol{u}_{-}\right) \cdot d \boldsymbol{x}=\int_{P}(\Omega \cdot \nu) d s
$$

где левая часть совпадает с циркуляцией $P_{+}-P_{-}$по контуру $P$, а правая часть представляет собой поток векторного поля $\Omega$ через границу поверхности, ограниченной контуром $P$, нормаль $\nu$ к которой связана с ориентацией контура правилом правого винта. Векторное поле $\Omega$ на $\Sigma$ назьвают также интенсивностью вихря (на единищу площади), а его траектории - вихревыми нитями пелены.

Из вьшесказанного следует, что

поверхность, при пересечении которой касательная компонента скорости резко меняется, является вихревой пеленой.

Основное аэродинамическое применение вихревых пелен касается поверхности и спутного следа крыла. Если $\boldsymbol{u}=\nabla \varphi(\omega=\nabla \wedge \boldsymbol{u}=0)$, то течение безвихревое (это следует, например, из несжимаемости, $\nabla \cdot \boldsymbol{u}=0$, отсутствия трения, $\mu=0$, и предположения об однородности приходяшего потока), однако предположение об отсутствии трения не выполняется в непосредственной близости поверхности крыла, и частицы 
жидкости завихряются вследствие трения. Таким образом, течение вблизи конечного несущего крыла является почти безвихревым всюду, кроме спутного следа, где завихренность должна присутствовать. Следует подчеркнуть, что сила сопротивления на крыле возникает из-за трения в жидкости или вязкости, однако ее величина благодаря вихревому следу не зависит от вязкости жидкости. Тем не менее толщина этого вихревого следа зависит от вязкости. Вязкость воздуха очень мала; в этом случае вихревой след является предельно тонким и обычно называется вихревой пеленой. Таким образом, мы можем исходить из простой идеи, заключаюшейся в том, что вихревая пелена представляет собой бесконечно узкую область с "бесконечной” завихренностью. Этому понятию можно придать строгий смысл в рамках теории обобщенных функций. А именно, завихренность пелены в заданной точке определяется как $\delta$-функция Дирака, умноженная на $\boldsymbol{n} \wedge[\boldsymbol{u}]$, где через

$$
[\boldsymbol{u}]=\boldsymbol{u}_{+}-\boldsymbol{u}_{-}
$$

обозначается разрыв скорости на пелене. Соответственно вектор

$$
\Omega(=n \wedge[u])
$$

назьвается "завихренностью пелены”.

Ниже мы будем рассматривать зависящий от времени, несжимаемьй, безвихревой поток в безразмерной математической постановке. Через $t, \boldsymbol{x}, \boldsymbol{u}$ и $p$ обозначаются соответственно время, пространственные координаты, скорость и давление, а плотность считается равной единище. Пусть функция $\chi(t, \boldsymbol{x})$ определена так, что пелена задается уравнением $\chi=$ const. Мы будем изучать следуюшую основную систему уравнений (отметим, что они не являются независимыми):

$$
\begin{gathered}
\nabla \cdot \boldsymbol{u}=0, \\
\frac{\partial \boldsymbol{u}}{\partial t}+(\boldsymbol{u} \cdot \nabla) \boldsymbol{u}+\nabla p=0, \\
\nabla \wedge \boldsymbol{u}=0, \\
\frac{\partial \chi}{\partial t}+\boldsymbol{u} \cdot \nabla \chi=0 \text { на обеих сторонах пелены, } \\
{[p]=\nabla \chi \cdot[\boldsymbol{u}]=0 \text { на пелене, }}
\end{gathered}
$$

где $[f]$ - скачок функции $f$ при переходе через пелену из области $\chi<$ const в область $\chi>$ const.

Рассмотрим тонкую вихревую нить (вихревая труба, поперечное сечение которой имеет инфинитезимальную максимальную размерность). Следуя работе Гиро и Зейтуняна [68], мы найдем асимптотическое представление ядра вихревой пелень, плотно намотанной на нить (так назьваемая свернувиаяся вихревая пелена).

Рассматриваемьй поток является безвихревым, однако имеет завихренность на пелене. Вихревая нить вместе с завихренностью, непрерьвно распределенной над трубчатой областью, представляет собой физическую модель свернувшейся вихревой пелены. Пусть $[\boldsymbol{u}]$ - разрыв скорости при пересечении пелены, а $h$ - расстояние между 
двумя последовательными витками. Если $\boldsymbol{n}$ - единичная нормаль к пелене, то из физических соображений следует, что

$$
\Omega=\frac{n \wedge[u]}{h}
$$

является хорошей аппроксимацией завихренности, распределенной над вихревой нитью, которая моделирует свернувшуюся пелену. Заметим, что разрыв $[\boldsymbol{u}]$ на пелене вычисляется в направлении нормали $\boldsymbol{n}$.

Эти рассуждения были формализованы в работе Гиро и Зейтуняна [68]. Здесь мы ограничимся коротким резюме этой статьи. Ее изложение с акцентом на физический смысл заинтересованный читатель может найти в [69].

Так как мы рассматриваем поток с очень большим числом близко расположенных вихревых пелен, каждая из которых является носителем слабой завихренности, то общее уравнение для этих пелен имеет вид $\chi(t, \boldsymbol{x})=(2 k+1) \pi, k=\ldots-2,0,1,2, \ldots$, а предположение об их близости выражается неравенствами

$$
|\partial \chi / \partial t| \gg 1 \text { и }|\nabla \chi| \gg 1 .
$$

Естественно, вся пелена задается на самом деле уравнением $\chi=$ const, однако из-за того, что она свернута вокруг нити, функция $\chi(t, \boldsymbol{x})$ является многозначной, и это объясняет, почему константа принимает множество значений. Вследствие введения функции $\chi$, ког да поток движется перпендикулярно поверхности $\chi=$ const, для любой из его переменных характеристик получаем модулированныц й пилообразныцй график.

Например, для скорости имеем

$$
\boldsymbol{u}=\boldsymbol{u}^{*}+\boldsymbol{u}_{1} Y(\chi)+\cdots,
$$

где $Y(\chi)$ - точная пилообразная функция:

$$
Y(\chi)=\chi \text { при }|\chi|<\pi \quad \text { и } \quad Y(\chi) \quad 2 \pi \text {-периодична. }
$$

Заметим, что

$$
[\boldsymbol{u}]=2 \pi \boldsymbol{u}_{1}
$$

при условии, что пелена пересекается в направлении роста $\chi$. Если обозначить теперь через $L$ характеристическую длину потока, так что, например,

$$
|\Omega|=O\left(\frac{|\boldsymbol{u}|}{L}\right),
$$

то из $(6.23),(6.27)$ и $(6.28)$ получим

$$
\left|\boldsymbol{u}_{1}\right|=O(C)|\boldsymbol{u}|,
$$


где $C=L / h$ - параметр близости. При этом если $C \ll 1$, то (6.25) можно интерпретировать как разложение относительно близости.

Как получить такое разложение из (6.22), подробно объяснено в [68]. Здесь мы только сформулируем результат, ограничиваясь разложением до порядка, указанного в (6.25). Еще один член вычислен в [68], однако здесь он нам не понадобится.

Основной результат состоит в следующем.

Рассмотрим вихревое решение $\left(\boldsymbol{u}^{*}, p^{*}\right)$ невязких несжимаемых уравнений Эйлера и такую функцию $\chi(t, \boldsymbol{x})$, что

$$
\frac{\partial \chi}{\partial t}+\boldsymbol{u}^{*} \cdot \nabla \chi=0 \text { и } \Omega^{*} \cdot \nabla \chi=0
$$

где $\Omega^{*}=\nabla \wedge \boldsymbol{u}^{*}-$ завихренность, ассоциированная с $\boldsymbol{u}^{*}$.

Предполагается, что функция $\chi$ неоднородна, имеет описанную выше структуру и удовлетворяет приведенным там условиям. Определим теперь порядок соотношения величин

$$
\frac{\left|\Omega^{*}\right|}{\left|\boldsymbol{u}^{*}\right||\nabla \chi|} \approx C
$$

и предположим, что $C \gg 1$. Тогда формулы

$$
\boldsymbol{u}=\boldsymbol{u}^{*}+\frac{\nabla \chi \wedge \Omega^{*}}{|\nabla \chi|^{2}} Y(\chi)+\cdots, \quad p=p^{*}+\cdots
$$

задают первые два члена двухмасштабного разложения относительно параметра близости С для безвихревого течения, содержсащего свернувшуюся вихревую пелену

$$
\chi(t, x)=(2 k+1) \pi, \quad k=\ldots-2,0,1,2, \ldots .
$$

Таким образом, наша (псевдо)математическая картина основана на алгоритме, которьй позволяет (достаточно убедительным, но не вполне строгим путем) сопоставить непрерывному вихревому течению с завихренностью, распределенной над некоторой областью, безвихревое разрывное течение с завихренностью, сосредоточенной на свернувшейся пелене. Разумеется, такое соответствие имеет место только в асимптотическом пределе, когда витки пелены бесконечно близки друг к другу.

В [68] установлено также, что для скачка потенциала скоростей на пелене имеют место следуюшие соотношения (где $\nabla_{T}$ - оператор градиента по касательным к пелене направлениям):

$$
\begin{gathered}
\nabla_{T} \Gamma=-2 \pi \frac{\nabla \chi \wedge \Omega^{*}}{|\nabla \chi|^{2}}, \\
\frac{\partial \Gamma}{\partial t}+\boldsymbol{u}^{*} \cdot \nabla \Gamma=0 .
\end{gathered}
$$

Эти соотношения обеспечивают возможность вычисления следующих членов разложения (условия согласования). Отметим, что (6.33b) - хорошо известное условие в 
теории вихревых пелен, и совсем не удивительно, что оно у нас также возникло. В работе Гиро [70] доказывается, что уравнение (6.33a) разрешимо относительно Г и что (6.33b) выполняется автоматически.

Заметим, что при исследования задачи о свернувшейся пелене можно ввести по крайней мере два мальх параметра, а именно, параметр удлинения, который для пелены с конической передней кромкой совпадает с расстоянием до фокуса спирали, и параметр близости, который представляет собой обратное к числу витков или расстояние между ними. Оказывается, что для вихревой пелены с конической передней кромкой второй параметр имеет порядок квадрата первого, если придерживаться стандартного предположения о том, что логарифмы - порядка единица. Именно через параметр удлинения внешнее решение влияет на разложение в ядре, и поэтому необходимо учитьвать отклонение от круговой симметрии. Приложение нашей теории к исследованию ядра пелень с передней кромкой, а также к так называемой задаче Кадена можно найти в [68; с. 100-109].

Однако хорошо известно, что вязкость рассеивает вихревые пелены. Для плоской пелены такое рассеяние описывается линейным уравнением диффузии. В работе Гиро и Зейтуняна [71] рассматривается вязкий случай Навье для свернувшейся вихревой пелены и доказывается, что вязкая диффузия завихренности, сосредоточенной на очень близко расположенных друг к другу витках пелены (плотно намотанная свернувшаяся вихревая пелена), также может быть описана линейным уравнением (теплопроводности), если соответствуюшим образом преобразовать функции $T$ (псевдовремя) и $\chi$. В этом случае диффузия является универсальньм процессом, при котором усредненньй поток $\boldsymbol{u}^{*}$ остается вязким и вихревым. Для того чтобы наблюдать влияние вязкости на усредненньй поток, необходимо рассматривать очень большие значения псевдовремени $T$, что эквивалентно переходу к глубоким слоям свернувшейся пелены в направлении оси течения в ядре. Описание рассеянного вязкостью непрерьвного течения в ядре можно найти в статье Стюартсона и Холла [72].

Наконец, в статье Гиро и Зейтуняна [73] результаты работы [68] о безвихревом несжимаемом невязком течении распространяются на случай вихревого сжимаемого течения Эйлера. Строго доказьвается, что в пределах ядра вычисления с пеленой можно обходить, определяя эквивалентное непрерьвное течение. Показано также, как завихренность и градиент энтропии между витками пелены переносятся вдоль траекторий эквивалентного течения.

Все эти результаты довольно прозрачны с физической точки зрения, и основной интерес нашей теории [68] состоит в том, что дается формальное доказательство их согласованности со стандартной схемой (асимптотического двухмасштабного) разложения относительно малого параметра близости.

Вихревая пелена и условие Кутты-Жуковского. Для тел с острой задней кромкой экспериментальные наблюдения дозвуковых течений показывают, что в общем случае благодаря вязкости течение вблизи задней кромки является гладким, и от задней кромки вниз по течению отстающими от поверхности тела слоями жидкости образуется тонкий спутньй след. Когда вязкость стремится к нулю, такая ситуация идеализируется предположениями о том, что спутньй след является бесконечно тонким с момента его отрыва от тела и что скорость на острой задней кромке конечна.

Последнее предположение называется условием Kутmы-ЖКковского в честь авторов, которые впервые его рассматривали (однако, согласно Гиро [74] имеются уст- 
ные свидетельства о том, что примерно в это же время аналогичная идея возникла у французского математика Вилля; читатель может найти намек на эту историю в [75], см. конец первой и начало второй страниц).

Мы будем предполагать, что условие Кутты-Жуковского налагается на острой задней кромке не только для дозвуковых, но и для сверхзвуковых течений; для сверхзвуковых течений это условие на задней кромке вьполняется автоматически [52]. В реальных течениях скорость в спутном следе быстро меняется, так что идеализированный спутный след можно рассматривать как вихревую пелену с момента его отрыва от задней кромки. Предполагается, что на некотором расстоянии от тела эта вихревая пелена под действием ее собственной индуцированной скорости сворачивается и на больших расстояниях вниз по течению принимает форму несовпадающих вихревых ядер конечного диаметра. Чем больше интенсивность вихревой пелены, тем быстрее она сворачивается.

Экспериментальные наблюдения в целом подтверждают такое предположение, однако под действием вязкости вихревые ядра в конце концов рассеиваются, так что на практике они не доходят до "бесконечности".

В линеаризованной теории условие Кутты-Жуковского формулируется следующим образом: компонента возмущенной скорости, перпендикулярная средней поверхности тела, конечна в окрестности любой задней кромки (обычное предположение о конечности скорости - слишком ограничительное для линеаризованной теории!). Как следствие, при математическом решении могут возникать поверхности, идущие от задней кромки вниз по течению, на которых касательная компонента скорости терпит разрыв.

Исчерпьвающее обсуждение условия Кутты-Жуковского в связи с так назьваемьм парадоксом Даламбера можно найти в статье Стюартсона [76].

На самом деле, согласно модели трехпалубной структуры и в противоположность принятой схеме Кутты-Жуковского (Вилля) течение не отрывается от поверхности в точности с задней кромки, а происходит небольшое его искривление с невязкой точкой торможения на подветренной стороне профиля на расстоянии порядка $O\left(\operatorname{Re}^{-3 / 8}\right)$ от задней кромки (см. [74; раздел 2.4, с. 269-271]).

Что касается теории трехпалубной структуры, отметим лишш, что она основана на отказе от асимптотической структуры, принятой в уравнениях пограничного слоя Прандтля. Она заменяется другой (локальной) структурой, из которой вьводится новая математическая (асимптотическая) модель.

Хороший обзор результатов, полученных в теории трехпалубной структуры, дан в работе Найфю [77]; однако обзорная статья Найфэ не включает наиболее свежие, интересные достижения! Отметим, что статья Мейера [78] проливает свет на многие вопросы.

В следуюшем, седьмом параграфе мы коротко рассмотрим модельные уравнения как для пограничного слоя Прандтля, так и для трехпалубной структуры.

\section{$\S$ 7. Корректные задачи}

При доказательстве существования, единственности, регулярности и устойчивости решений задач гидродинамики необходимо придать смысл уравнениям Навье-Стокса и соответствующим начальным и граничным условиям. 
При любом разумном выборе класса допустимых начальных функций $\left[\boldsymbol{u}^{0}(\boldsymbol{x})\right.$, $\left.\rho^{0}(\boldsymbol{x}), T^{0}(\boldsymbol{x})\right]$ задача Навье-Стокса о течении является корректной (в смысле Адамара [1]). Это означает, что

(a) для любы $x$ начальных функций $\left(\boldsymbol{u}^{0}(\boldsymbol{x}), \rho^{0}(\boldsymbol{x}), T^{0}(\boldsymbol{x})\right)$, принадлежаших выбранному классу, задача имеет решение;

(b) решение единственно для любых заданных начальных условий;

(c) решение непрерывно зависит от начальных данных.

Последнее свойство означает, что если возмушение $\delta U$ решения $U(t)$ мало в начальный момент $t=0$, то оно мало для любого $t>0$ (условие устойчивости). Более точно, для каждого конечного интервала $\left[0, t_{0}\right]$ найдется такая константа $K=K\left(t_{0}\right)$, что любое классическое решение (сильное решение начальной задачи) уравнений Навье-Стокса удовлетворяет неравенству

$$
\|U(t)\| \leqslant K\left\|U\left(t_{0}\right)\right\| \text { при } 0 \leqslant t \leqslant t_{0} .
$$

Естественно, вопрос о корректности задачи может зависеть от выбора нормы. Задачи, не удовлетворяюшие хотя бы одному из вышеперечисленных свойств (а)-(c), называются некорректныцми. Следующее условие является необходимым для устойчивости решений.

Малая ошибка или малое возмущение начального состояния приводит к малому изменению конечного состояния, т.е. решение непрерьвно зависит от начальных данных.

Адамар, в частности, подчеркивал важность непрерьвной зависимости решений от начальных данных (свойство (c)), так как практические задачи (в гидродинамике, метеорологии, и т. д.) нередко приводят к некорректным постановкам. Начальная задача некорректна, даже если решение сушествует, но оно не зависит непрерьвно от начальных данных. Некорректность - более сильное свойство, чем просто неустойчивость. Но удивительно то, что некорректная задача может представлять физический интерес. К сожалению, для уравнений Навье-Стокса реальные задачи чаше оказываются математически некорректными, и в поведении решений проявляется ключевое свойство, называемое “чувствительной зависимостью от начальных условий” (ЧЗНУ) и представляюшее собой важную характеристику турбулентности (xaoca!).

На самом деле нелинейные задачи Навье-Стокса имеют много сложных особенностей, таких как явления бифуркации, турбулентность (хаос) и тепловой перенос.

Например, задача о переносе по характеру отличается от начальной задачи, так как непрогнозируемость деталей - сушественная особенность.

Проблемы динамической метеорологии также представляют собой гидродинамическую задачу (см., например, недавнюю книгу автора [79]). Эти метеорологические задачи имеют промежуточную природу. В таких задачах случайные эффекты и неадекватное знание начальных данных приводят к невозможности долгосрочных прогнозов, однако со временем новые данные становятся доступными из наблюдений.

Нелинейные эффекты многочисленны и разнообразны, и поэтому трудно ожидать, что какая-то дисциплина, скажем гидродинамика, может проиллюстрировать все такие явления! Однако, ввиду сложности и разнообразности гидродинамических явлений и (относительной!) простоты и точности управляющих ими уравнений можно 
ожидать, что математическая теория будет очень глубокой и красивой. То, что многие важные задачи остаются до сих пор нерешенными, может служить источником вдохновения и стимулировать создание глубокой, оригинальной и действенной теории в будущем; обсуждение некоторых из этих важных вопросов можно найти в статье Хейвуда [80].

Интересно отметить, что задачу о гидродинамической устойчивости можно интерпретировать как начальную задачу, так как классический метод разложения по собственным модам неявно игнорирует начальную фазу роста искажения или возмущения основного состояния (или течения). Может показаться, что результаты, полученные методом разложения по собственным модам, можно использовать для построения решения начальной задачи, представляя его, по крайней мере формально, в виде суперпозиции таких мод (в линейном случае!). Однако практически это не так просто, так как переходньй режим трудно описать из-за того, что при $t \rightarrow 0$ число значимых мод увеличивается. Более того, даже для формального применения этой процедуры имеется существенное препятствие (см., например, статью Бергера [81]), состоящее в том, что спектр возмущения является непрерьвным, а не дискретным, и поэтому переходньй режим нельзя описать линейной суперпозицией бесконечного числа дискретных мод.

В литературе эта трудность обходится рассмотрением только наименее демпфированной из собственных мод и прогнозированием появления разрыва, когда эта мода увеличивается в $e$ раз. Однако из-за того, что приближение одной собственной модой эффективно лиш в асимптотическом пределе (при $t \rightarrow \infty)$, прогноз времени появления разрьва может оказаться ошибочным; в самом деле, возмушение может увеличиваться больше чем в $е$ раз еше до того, как приближение становится эффективным.

Приведенные выше рассмотрения были основной мотивацией для (успешного) применения этих идей к исследованию важных задач об устойчивости, а также о волнах и осциллящиях малой амплитуды.

Корректность двумерного идеального течения. В статье [82] дается обзор математических результатов, касаюшихся корректности уравнений Эйлера для идеальной (несжимаемой невязкой) жидкости в двумерном случае. Если в начальньй момент времени завихренность ограничена (в гёльдеровской норме), то существует единственное решение, определенное при всех временах и обладающее такой же гладкостью, как и начальные данные. Однако это условие не выполняется в классическом контексте неустойчивости Кельвина-Гельмгольиа, когда начальная скорость терпит разрьв на вихревой пелене! В этом случае существование вихревой пелены при мальх временах гарантируется только аналитичностью пелены и плотности завихренности в начальный момент времени. Асимптотический анализ (Мур [83]) и численное моделирование подсказывают, что за конечное время в вихревой пелене образуются особенности. Поэтому глобальное существование течения имеет место лишь в слабом смысле. Так или иначе анализ Гиро и Зейтуняна [84] показывает, что задачи устойчивости для свернувшихся пелен, в принципе, не являются локальньми. Однако, когда длины волн пренебрежимо малы по сравнению с расстоянием (между витками пелены), механизм неустойчивости такой же, как и в случае бесконечного пилообразного профиля, и это подтверждает, что время роста имеет порядок $O\left(\lambda / C\left|\boldsymbol{u}^{*}\right|\right)$, когда $\lambda$ мало по сравнению с $D /\left|\boldsymbol{u}^{*}\right|$, где $D$ - диаметр ядра. С другой 
стороны, при $\lambda=O\left(D /\left|\boldsymbol{u}^{*}\right|\right)$ анализ устойчивости должен учитывать всю свернувшуюся вихревую пелену!

Прежде всего отметим, что в трехмерном случае, какими бы гладкими ни были начальные условия, существование единственного (классического) несжимаемого решения уравнений Эйлера установлено только на конечном интервале $\left[0, t^{*}[\right.$, длина которого обратно пропорциональна гёльдеровской норме начальной завихренности. На этом промежутке решение обладает такой же гладкостью, как и начальное условие; если начальные данные аналитичны, то решение также аналитично (см. обзор [85]). До сих пор не известно, является ли задача корректной в слабом смысле при $t>t^{*}$.

Положение намного лучше в двумерном случае благодаря сохранению завихренности $\omega$, которая отождествляется с вектором, перпендикулярным плоскости течения, и удовлетворяет классическому уравнению вихря:

$$
\frac{\partial \omega}{\partial t}+(\boldsymbol{u} \cdot \nabla) \omega=0
$$

В самом деле, классическое решение сушествует и единственно при всех временах, если начальная завихренность непрерывна по Гёльдеру. Геометрическое доказательство этого утверждения, данное в работе Эбина и Марсдена [86], использует тот факт, что движение идеальной несжимаемой жидкости представляет собой геодезический поток на бесконечномерном многообразии сохраняющих объем диффеоморфизмов.

Имеется конструктивная процедура, доказьвающая глобальное существование решения начальной задачи Эйлера в двумерной области $D$ :

$$
\begin{gathered}
\frac{\partial \boldsymbol{u}}{\partial t}+(\boldsymbol{u} \cdot \nabla) \boldsymbol{u}+\nabla p=0 \\
\nabla \cdot \boldsymbol{u}=0 \\
\boldsymbol{u} \cdot \boldsymbol{n}=0 \text { на } \partial D \\
\boldsymbol{u}(0, \boldsymbol{x})=\boldsymbol{u}^{0}(\boldsymbol{x})
\end{gathered}
$$

Для существования классического решения уравнений Эйлера требуется, чтобы скорость и градиент скорости были ограниченъ. Легко показать, чтовсепроизводные векторного поля, ограниченные в начальньй момент времени, остаются ограниченными. Для неограниченной области необходимо, чтобы начальные условия достаточно быстро убывали на бесконечности, что обеспечивает абсолютную интегрируемость завихренности. Предположение об абсолютной интегрируемости начальной завихренности в неограниченной области обшего вида связано с возможньм медленным убыванием на бесконечности ротора функции Грина соответствуюшего уравнения Пуассона.

Единственность классического решения легко следует из свойств регулярности.

Таким образом, для сушествования (единственного) классического решения уравнений Эйлера необходимо, чтобы начальная завихренность была непрерывна по Гёльдеру. Однако, когда это условие не выполняется, можно ввести понятие слабого решения (в смысле обобшенных функций), так как уравнения Эйлера записываются в виде закона сохранения.

Классический пример сингулярного решения уравнений Эйлера соответствует неустойчивости Кельвина-Гельмгольца, когда начальная скорость терпит разрьв на 
гладкой кривой, на которой сосредоточена завихренность с гладкой линейной плотностью. В этом случае, если полная энергия течения конечна, а линейная плотность завихренности абсолютно интегрируема на поверхности, то сушествует слабое решение уравнений Эйлера [87].

Подчеркнем еще раз, что в области, неограниченной в двух направлениях, мы не можем установить корректность задачи без предположения об убьвании решения на бесконечности.

Что касается нелинейных длинных волн на воде и солитонов, недавняя обзорная статья [88] содержит различные математические результаты о существовании, единственности и устойчивости решения классической задачи.

Неустойчивости Кельвина-Гельмгольца и Тейлора. Чтобы начальная задача была корректной в смысле Адамара, необходимо не только существование единственного решения для всех начальных состояний системы, определяемых некоторым "разумньм" классом начальных функций, но требуется еще непрерьвная зависимость решения от начальных данных в соответствуюшем смысле.

Неустойчивость Гельмгольца означает, что в некоторый момент времени зависимость от начальных данных не является непрерывной. Предположим, что плоскость разбивает объемлющее пространство на две области (полупространства), в каждой из которых равномерно движется жидкость, причем скорости движения различны, а на самой плоскости жидкость скользит (без трения). Введя малое возмушение начальных данных путем перехода от плоскости скольжения к волнистой поверхности малой синусоидальной амплитуды и соответствующего незначительного изменения течения вблизи этой поверхности, можно получить решение, в котором амплитуда возмушения со временем возрастает; этот рост является әкспоненииальным до тех пор, пока амплитуда мала по сравнению с длиной волны, т.е. для линеаризованной задачи (см. ниже).

Описанное вьше явление служит причиной, например, образования волн на поверхности водоема в результате слабого берегового ветра; это явление известно как неустойчивость Гельмгольиа. Естественно, при этом предполагается, что скорость скольжения не превосходит определенной части скорости звука: при больших относительных скоростях акустические эффекты подавляют неустойчивости. Более того, скорость экспоненциального роста такого возмушения неограниченно возрастает, когда длина волны $\lambda$ первоначальных колебаний стремится к нулю.

Следовательно, для любъх $\varepsilon>0$ и $M>0$ существует такое достаточно малое $\lambda$, что начальное "инфинитезимальное" возмущение с длиной волны $\lambda$ возрастает более чем в $M$ раз за промежуток времени, меньиий, чем $\varepsilon$, т.е. зависимость решения линеаризованной задачи от начальных данных не является непрерывной.

Однако если между двумя жидкостями сушествует поверхностное натяжение (граничное натяжение), то возмушения с длиной волны, меньшей некоторого $\lambda_{0}$, не возрастают, и поэтому непрерывная зависимость от начальных данных восстанавливается, даже если возмушения с большей длиной волны остаются неустойчивыми. Волны на поверхности воды стабилизируются под действием поверхностного натяжения и гравитации (введение в теорию волн на воде можно найти в книге Уизема [89; гл. 13]).

С другой стороны, когда поверхность скольжения образована внутри данной жидкости (например, в результате движения аэродинамической поверхности или пересе- 
чения двух ударных волн в задаче Маха об отражении), стабилизируюшие факторы, вообще говоря, отсутствуют.

Согласно Рихтмайеру [19; т. I, раздел 17.15] предыдущие соображения основаны на линеаризованной теории неустойчивости. Предположим, что невозмушенная поверхность совпадает с плоскостью $(x, y)$, а возмушенная поверхность $S$ задается уравнением $z=\zeta(t, x, y)$.

Линеаризованная теория основана на предположении, что производные $\partial \zeta / \partial x$ и $\partial \zeta / \partial y$ всюду пренебрежимо малы по сравнению с 1 (более точная линейная теория развита в [90; с. 267-268]).

Для простоты мы не будем учитьвать влияние гравитации, поверхностного натяжения и сжимаемости (более полный и реалистичный подход к асимптотической нелинейной теории волн на воде можно найти в [88] и [90]) и будем предполагать, что течение является безвихревым по обе стороны поверхности $S$, так что в любой момент времени $t$ скорость выражается через свой потенциал $\varphi$ по формуле

$$
\boldsymbol{u}=\nabla \varphi, \quad \text { где } \nabla^{2} \varphi=0 \text { в любой области. }
$$

Тогда начальная задача формулируется следующим образом:

$$
\zeta(t, x, y) \text { и } \frac{\partial \zeta}{\partial t}(t, x, y) \text { заданы при } t=0 \text { как функции от } x \text { и } y \text {; }
$$

в каждой области потенциал скорости $\varphi(t, x, y, z)$ определяется при любом $t$ по функциям (7.5) с помощью уравнения Лапласа $\nabla^{2} \varphi=0$ и следующих условий:

(a) нормальная компонента скорости $\boldsymbol{u}$ непрерывна при переходе через $S$ и согласована на $S$ с нормальной компонентой скорости самой поверхности $S$;

(b) вектор $\boldsymbol{u}$ стремится к $( \pm V / 2,0,0)$ при $z \rightarrow \pm \infty$, где $V$ - относительная скорость или скорость скольжения.

Важно отметить, что потенциал скорости $\varphi$ не является непрерывным при переходе через $S$, и движение определяется из условия, что давление $p$ непрерывно на $S$ и удовлетворяет уравнению Эйлера:

$$
\rho_{0}\left(\frac{\partial}{\partial t}+\boldsymbol{u} \cdot \nabla\right) \boldsymbol{u}=-\nabla p .
$$

Рассмотрим решение задачи, в котором

$$
\zeta(t, x, y)=\sum_{k=-\infty}^{+\infty} c_{k}(t) e^{i(k x+l y)}
$$

В линейном приближении каждое слагаемое можно изучать отдельно, и для функций $c_{k}$, отвечаюших за зависимость от времени, получаем формулы

$$
c_{k}(t)=A_{k} e^{k V t / 2}+B_{k} e^{-k V t / 2},
$$

которые справедливы до тех пор, пока амплитуда мала по сравнению с длиной волны. 
Коэффициент в одном из показателей, а именно $\frac{1}{2}|k| V$, стремится к бесконечности при $k \rightarrow+\infty$; поэтому для сходимости ряда

$$
\sum_{k=-\infty}^{+\infty} d_{k} \exp \left[i(k x+l y)+\frac{1}{2}|k| V t\right]
$$

при некотором $t=t_{0}>0$ необходимо, чтобы

$$
d_{k}=o\left(\exp \left[-\frac{1}{2}|k| V t_{0}\right]\right) \text { при } k \rightarrow+\infty,
$$

откуда следует, что начальная поверхность должна быть аналитической: если она не является аналитической, то решение нельзя построить методом рядов Фурье!

Поведение поверхности раздела при больших амплитудах (когда линейная аппроксимация не пригодна) изучалось экспериментально и численным моделированием.

Для поверхности, которая в начальньй момент времени имела вид $\zeta=a_{k}(t) \cos k x$ (одномерная волна), установлено, что когда амплитуда становится сравнимой с длиной волны, т.е. когда $a_{k}(t) \approx 1 / k$, рост $a_{k}(t)$ является уже не экспоненциальным, а приближенно линейньм, причем $d a_{k}(t) / d t \approx \alpha V$, где $\alpha$ - константа порядка единицы.

Зависимость от $x$ (и $y$ ) также меняется. Наблюдения показывают, что при еще больших амплитудах $\left(a_{k}(t) \gg 1 / k\right)$ поверхность разрушается и заменяется турбулентнылм слоем, толшина которого со временем возрастает из-за вихревой диффузии. Мелкие начальные иррегулярности любого сорта приводят к немедленному локальному разрушению поверхности.

С другой стороны, если начальная поверхность является кусочно-аналитической, скажем, с зигзагообразным поперечным сечением, и если справедливо следуюшее предположение:

если начальные данные кусочно-аналитичны, то, по крайней мере, на некотором конечном интервале времени существует единственное кусочно-аналитическое решение,

то должно существовать кусочно-аналитическое решение, однако природа этого решения неизвестна.

Неустойчивость Тейлора имеет аналогичный характер. В этом случае предполагается, что нет скольжения вдоль поверхности $S$, но жиидости имеют различные плотности, а система испытьвает ускорение в направлении от тяжелой жидкости к легкой. Примером такой системы, в которой ускорение заменено эквивалентным гравитационным полем, служит вода в верхней части сосуда, поддерживаемая давлением воздуха, находящегося в нижней части. Если поверхность раздела - в точности горизонтальная плоскость, то система находится в равновесном, но неустойчивом состоянии, при котором волны на поверхности имеют зависящий от времени множитель вида $\exp ( \pm \alpha t)$, а не $\sin \alpha t$ или $\cos \alpha t$, как для обьчных волн на воде. Снова коэффициент $\alpha$ неограниченно возрастает (если поверхностное натяжение отсутствует), когда длина волны убывает.

Интересно отметить, что корректность задачи о вихревой пелене (для несжимаемого невязкого безвихревого течения) на конечном интервале времени с аналитическими данньпи, впервые предсказанная Биркгофом (в 1962 году) в размерности два, 
имеет место как в двумерном, так и в трехмерном случаях [91]. В двумерном случае установлено глобальное существование слабого решения уравнений Эйлера с аналитическими начальными условиями. В трехмерном случае получено лагранжево представление вихревой пелены, аналогичное уравнению Биркгофа в размерности два, и для описания эволюции неустойчивости и возможного появления особенностей было бы интересно получить представление вихревой пелены, допускающее сворачивание границы. На самом деле показано, что особенность появляется за конечное время, что согласуется с асимптотическим результатом Мура [83].

Наконец, касаясь неустойчивости Кельвина-Гельмгольца, отметим, что нелинейная эволюция такой неустойчивости изучалась в работах Найфэ и Сарика [92] и [93] в случае, когда амплитуда неустойчивой волны однородна по пространственным переменньм и растет только по времени. В статье Вайсмана [94] изучаются эволюция и распространение волновых пакетов, представляющих собой волновой поезд, в котором амплитуда зависит как от пространственных переменных, так и от времени. С помощью метода кратного скейлинга получено обобщение амплитудного уравнения, которое пригодно как для устойчивых, так и для маргинально неустойчивых областей пространства параметров.

Парадокс Даламбера. Согласно статье Стюартсона [76] математическую гипотезу, ассоциированную с парадоксом Даламбера, наиболее ясно можно сформулировать в терминах уравнений Навье для несжимаемой вязкой жидкости, которые в случае стационарного движения записываются в виде

$$
-\boldsymbol{u} \wedge \omega=-\nabla\left(\frac{p}{\rho_{0}}+\frac{\boldsymbol{u}^{2}}{2}\right)+\mu \nabla^{2} \boldsymbol{u}, \quad \nabla \cdot \boldsymbol{u}=0 .
$$

Парадокс Даламбера возникает в результате наложения интуитивно разумных предположений о том, что при больших числах Рейнольдса вязким членом $\left(\mu / \rho_{0}\right) \nabla^{2} \boldsymbol{u}$ можно пренебречь, условие непроскальзывания можно заменить требованием касания, а полученное решение является гладким. Однако в этом случае управляюшие уравнения переходят в уравнения Эйлера, и имеет место теорема Бернулли (если наложенные условия выполняются равномерно на бесконечном расстоянии от тела):

$$
\frac{p}{\rho_{0}}+\frac{\boldsymbol{u}^{2}}{2}=\text { const }
$$

При этом, как можно строго показать,

сопротивление среды отсутствует, что в точности совпадает с выводом Даламбера!

Следовательно, проекция действуюшей на тело силы на направление основного потока $\boldsymbol{i}$ не изменится, если заменить $\boldsymbol{i}$ на $-\boldsymbol{i}$. Таким образом, если для какого-то течения сопротивление среды положительно, то оно станет отрицательным, если на бесконечности изменить направление движения жидкости на противоположное. Это противоречит второму закону термодинамики!

Легко представить себе смятение, возникающее в результате такого заключения, полностью противоречащего ежедневным наблюдениям, если к тому же учесть, что 
уменьшение сопротивления на движущееся тело является основной проблемой для специалистов по прикладной гидродинамике, таких как проектировщики кораблей и инженеры по авиационной технике. Описанньй вьше парадокс преследовал теоретиков в девятнадцатом веке и раньше, но они постепенно смирились с ним. Несмотря ни на что, теория может внести сушественный вклад в понимание движения тел в жидкости.

Так как классическая невязкая теория приводит к безусловно абсурдному выводу, согласно которому сопротивление, испытываемое твердым телом при равномерном движении в жидкости, равно нулю, за последние сто лет были предприняты серьезные попытки построить альтернативную теорию и объяснить, как пренебрежимо малая сила трения в жидкости может иметь существенное влияние на свойства течения. Используемые при этом методы представляют собой комбинацию экспериментальных наблюдений, вычислений, проводимых часто на очень большой шкале, и анализа структуры асимптотического вида решения, когда трение стремится к нулю. Эти исследования имели значительньй успех, особенно за последние тридцать лет, так что теперь парадокс можно считать в значительной степени решенным.

Целью теоретических исследований является получение правильной структуры решения уравнения (7.10), когда число Рейнольдса Re $=U_{\infty} L / \nu$ стремится к бесконечности (где $\nu=\mu / \rho_{0}, L$ - характерная длина заданного тела с гладкой границей, a $U_{\infty}$ - скорость равномерного движения на бесконечном расстоянии от тела в направлении, определяемом единичным вектором $\boldsymbol{i}$ ), что позволит понять парадокс Даламбера. В самом деле, жидкость, движущаяся вблизи плохо обтекаемого тела, в передней точке торможения не остается так же близко к телу, как и в задней точке торможения, а отрывается от поверхности, оставляя сзади вихревой след с почти постоянньп давлением. Этот след, который может распространяться на большие расстояния сзади от тела, является турбулентным, но мы можем заключить, что в соответствующем ламинарном решении жидкость также отделяется от тела, порождая поверхность разрьва. По одну сторону от этой поверхности жидкость берет начало на бесконечном расстоянии от тела в направлении, противоположном ее движению, тогда как по другую сторону она имеет иное происхождение. Простейшее предположение, которое можно налагать на жидкость во второй области, состоит в том, что она находится в покое, образуя так называемую застойную зону в течении. Поэтому имеются два возможных кандидата для предельного решения уравнений Навье (7.10), один из которых ( соединенное потенциальное течение) является гладким и приводит к парадоксу Даламбера, а другой (так назьваемое свободное ламинарное течение Кирхгофа; см., например, книгу Биркгофа и Сарантонелло [95]) является разрьвным и имеет некоторую асимметрию, позволяющую избегать парадоксов.

Так как мы допускаем наличие разрьвов, необходимо ответить на многие вопросы, уточняющие постановку задачи.

Как выбирается точка отрыва? Находится ли жидкость в покое в спутном следе? Сколько имеется точек отрыва? Является ли спутный след конечным?

На эти вопросы невозможно ответить в рамках невязкой теории!

Следуюший важньй шаг был сделан Прандтлем (1904 г.), который ввел понятие пограничного слоя.

Пограничный слой Прандтля. Математически, когда $\operatorname{Re} \rightarrow \infty$, невязкое решение не может сушествовать всюду, так как оно не удовлетворяет условию непроскальзьва- 
ния на границе тела. Единственньй способ устранения этой неравномерности - ввести (тонкий) слой вблизи тела, где вязкость имеет сушественное влияние. К счастью, толщина этого слоя стремится к нулю при $\operatorname{Re} \rightarrow \infty$.

В простейшей форме (для двумерного стационарного несжимаемого течения) эта теория предполагает, что невязкое течение было правильно вычислено и что (горизонтальная) скорость скольжения на границе тела равна $u_{e}(x)$, причем считается, что граница задается (в данном случае) уравнением $y^{*}=0$. Скорость скольжения обрашается в нуль на границе тела и в полосе ширины порядка $O\left(L / \mathrm{Re}^{1 / 2}\right)$. Система уравнений для этого тонкого слоя получается в результате скейлинга компоненты скорости $v^{*}$ (перпендикулярной к границе тела) с коэффициентом $\mathrm{Re}^{-1 / 2}$, так что если $\left(x^{*}, y^{*}\right)$ обозначает расстояния по касательному и нормальному к гранище тела направлениям, а $\left(u^{*}, v^{*}\right)$ - соответствующие компоненты скорости, то в пограничном слое имеем

$$
y^{*}=y \operatorname{Re}^{-1 / 2}, \quad x^{*}=x, \quad v^{*}=v \operatorname{Re}^{-1 / 2}, \quad u^{*}=u, \quad p^{*}=p .
$$

В этом случае для функций $u, v$ и $p$ переменных $x$ и $y$ уравнения Навье сводятся к приводимым ниже уравнениям Прандтля, которые получаются в результате подстановки соотношений (7.12) в (7.10) и перехода к пределу, когда $\operatorname{Re} \rightarrow \infty$ при фиксированных $x$ и $y$ :

$$
\begin{gathered}
\frac{\partial u}{\partial x}+\frac{\partial v}{\partial y}=0 \\
u \frac{\partial u}{\partial x}+v \frac{\partial u}{\partial y}=-\frac{1}{\rho_{0}} \frac{\partial p}{\partial x}+\frac{\partial^{2} u}{\partial y^{2}} \\
\frac{\partial p}{\partial y}=0 .
\end{gathered}
$$

Таким образом, в пограничном слое давление постоянно и совпадает с его значением на внешней границе этого слоя (см. (7.11)): $\left(1 / \rho_{0}\right) \partial p / \partial x=u_{e}\left(\partial u_{e} / \partial x\right)$.

Для непроницаемой твердой стенки задаются соответствующие граничные условия

$$
u=v=0 \text { при } y=0, \quad u \rightarrow u_{e} \text { при } y \rightarrow \infty,
$$

а также некоторые условия на $u$ в начальной точке $x$, которые обычно имеют вид

$$
u=0 \text { при } y>0 \text { в передней точке торможения } x=x_{0} \text {. }
$$

В настоящее время известно, что при этих условиях решение системы уравнений (7.13) сушествует в некоторой области $x_{0}<x<x_{1}$, если $\partial u_{e} / \partial x \geqslant 0$ (Олейник [96]), и единственно до тех пор пока $u>0$ (Никел [97]). Прандтль предположил, опираясь частично на наблюдения, что основное течение отрывается от поверхности, когда коэффициент поверхностного трения

$$
\tau(x)=\left(\frac{\partial u}{\partial y}\right)_{y=0}
$$


обрашается в нуль, что имеет место при отрицательном градиенте давления: $\partial u_{e} / \partial x<0$. В некотором смысле это условие определяет положение точки отрывв.

Несмотря на то, что результаты Прандтля были определенным шагом вперед и, кроме того, представляли большой интерес в аэронавтике, оставались открытыми многие проблемы, в частности, вопросы о том, почему обращение в нуль функции $\tau(x)$ является определяющим фактором и каков механизм, благодаря которому свободное ламинарное течение покидает окрестность тела! На самом деле, когда $\tau(x)=0$, утверждение, что пограничный слой имеет только небольшое влияние на невязкое течение вне слоя, является не совсем точным. По крайней мере, невязкое течение должно быть существенно модифицировано вблизи точки $x=x_{s}, y=0$, для которой $\tau\left(x_{s}\right)=0$. Прандтль указьвал, что согласно экспериментальным наблюдениям невязкое течение покидает окрестность тела именно в этой точке, и ввел понятие отрьва для описания указанного явления. Гольдштейн [98] показал, что решение уравнений пограничного слоя не может быть продолжено вниз по течению в направлении отрьва, если появляется особенность и тем самым происходит полное разрушение иерархической системы.

В данном случае, по-видимому, невозможно ограничить влияние особенности окрестностью точки отрыва, используя рассуждения, основанные на эффекте трехпалубной структурь (см. статью Стюартсона [99]). Краткий обзор асимптотик, возникающих в теории трехпалубной структуры, можно найти в [74; с. 262-271].

По-видимому, схема, согласно которой отрыв течения от гладкой поверхности (Сычев [100]) возникает в результате отрицательного градиента давления на конечном участке поверхности тела, в действительности вообще не имеет места [76; с. 312]!

Классическое безвихревое невязкое присоединенное течение вокруг тела плохо обтекаемой формы не может быть правильным пределом течения Навье-Стокса при $\operatorname{Re} \rightarrow \infty$. Это утверждение устраняет основную концепцию, приводящую к парадоксу Даламбера. В самом деле, необходимо ответить на следующие два вопроса: можно ли построить внешнее распределение скоростей, которое не приводит к особенностям, и как пограничный слой обеспечивает необходимое для этого ограничение?

Ответ на первый вопрос, данный в работе Катералла и Манглера [101], состоит в том, что не надо задавать градиент внешнего давления. Как следствие, при отрьве не возникают особенности, связанные с толщиной вытеснения.

Ответ на второй вопрос дается в рамках теории трехпалубной структуры, независимо развитой в работах Нейланда [102] и Стюартсона и Уильямса [103]. Всесторонний обзор результатов, полученных применением этой концепщии, можно найти в [77] и $[78]$.

Трехпалубная структура. Имеется много модификаций асимптотической структуры (Прандтля), но одна из них является особенно значимой, так как приводит к новой, по-видимому, очень богатой асимптотической структуре, и именно в результате этой модификации возникает трехпалубная структура. Выберем какую-нибудь точку, скажем $x_{a}$, и положим $x-x_{a}=O\left(\mathrm{Re}^{-3 / 8}\right)$. Порядок $\mathrm{Re}^{-3 / 8}$ получен экспериментально, методом проб и ошибок (однако такой же порядок получается в результате анализа теории неустойчивости, а также из решения Лайтхилла (1953 г.) парадокса передачи возмушений вниз по потоку). Отметим, что Маусс [104] указал дедуктивньй способ получения этого порядка. Выбрав асимптотический порядок продольного размера, находим, что в нормальном направлении к стенке должна быть трехслойная струк- 
тура. Имеются три разных уравнения, зависящих от порядка нормальной координаты $y$, а именно, рассматриваются "нижний подслой", для которого $y=O\left(\operatorname{Re}^{-5 / 8}\right)$, “основной подслой”, для которого $y=O\left(\operatorname{Re}^{-1 / 2}\right)$, и “верхний подслой”, для которого $y=O\left(\operatorname{Re}^{-3 / 8}\right)$.

При этом классический пограничный слой Прандтля (Блазиуса) разбивается на две части, которые обычно назьваются нижним и основным подслоями. Третий подслой представляет собой область внешнего течения, на которую быстрые изменения пограничного слоя влияют наиболее существенным образом. Этот подслой называется верхним и расположен прямо над двумя другими подслоями (см. приводимьй ниже рисунок).

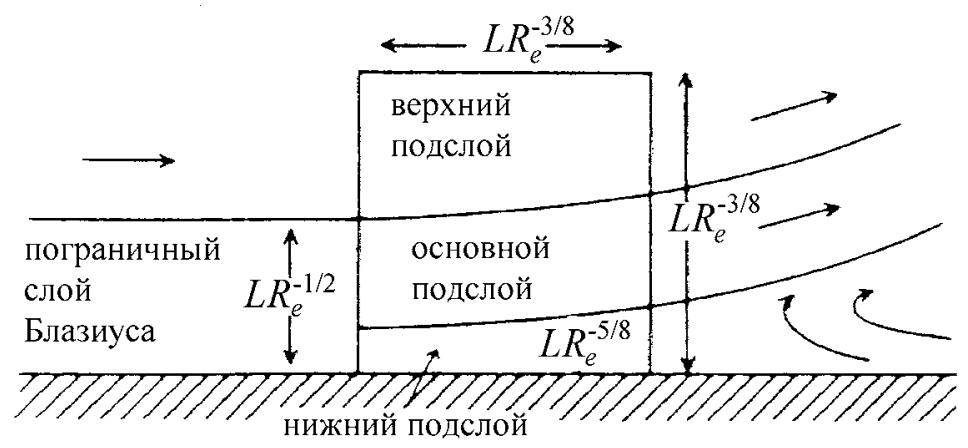

Рис. 1. Эскиз области трехпалубной структуры около отрыва на плоской пластине

В нижнем подслое классические уравнения пограничного слоя Прандтля (7.13) выполняются с точностью до старшего порядка, однако их (асимптотический) вывод требует применения разных скейлингов (для компонент скорости и давления), которые мы не будем уточнять. Условия непроскальзьвания вьполняются на стенке, и вверх по течению от области трехпалубной структуры решение должно быть согласовано с обычным пограничным слоем, от которого оно эволюционирует. Следовательно,

$$
u \rightarrow \lambda y \text { при } x \rightarrow-\infty \quad \text { и } \quad u-\lambda y \rightarrow \lambda A(x) \text { при } y \rightarrow \infty,
$$

где согласно обычной теории $\lambda$ - значение коэффициента поверхностного трения $\tau$ в начале координат трехслойной области, а $A(x)$ - неизвестная функция, которая определяется с помощью давления $p$.

В некотором смысле теория основного подслоя играет пассивную роль, передавая эффективную скорость скольжения $\lambda A(x)$ через пограничный вязкий нижний подслой и преобразовывая ее в скорость по нормали на внешней границе, однако это сушественно для успешного применения схемы. Если основной пограничный слой Прандтля имеет профиль $U_{b}(y)$ в области взаимодействия, то из (7.10) получим уравнения

$$
u^{*}=U_{b}(y)+A(x) \frac{d U_{b}(y)}{d y}, \quad v^{*}=-\operatorname{Re}^{-1 / 2} \frac{d A(x)}{d x} U_{b}(y)+\cdots
$$


которые выполняются в основном подслое и приводят к автоматической согласованности между $x$-компонентами скорости в основном и нижнем подслоях. Если $y \rightarrow \infty$ в масштабе основного подслоя, то

$$
u^{*} \rightarrow U_{b}(\infty), \quad v^{*} \rightarrow-\operatorname{Re}^{-1 / 2} \frac{d A(x)}{d x} U_{b}(\infty),
$$

так что малый параметр $\mathrm{Re}^{-1 / 2}$ в скорости по нормали на внешней границе пограничного слоя частично компенсируется усиливающим влиянием производной по $x$. Скорость по нормали действует на основание верхнего подслоя и порождает градиент давления порядка $O\left(\operatorname{Re}^{-1 / 2}\left[d^{2} A(x) / d x^{2}\right]\right)$ в безразмерных величинах, что в свою очередь управляет нижним подслоем. Для двумерного несжимаемого внешнего течения уравнение трехпалубной структуры совпадает с (эллиптическим) уравнением Лапласа, и функциональное соотношение, связываюшее $p(x)$ и $A(x)$, задается интегралом Гильберта:

$$
p=-\frac{1}{\pi} \int_{-\infty}^{\infty} \frac{d A\left(x^{\prime}\right) / d x^{\prime}}{x-x^{\prime}} d x^{\prime}
$$

Наконец, для твердой непроницаемой стенки соответствующие условия для нижнего подслоя имеют вид

$$
u=v=0 \text { при } y=0 \text {, }
$$

причем для этого нижнего подслоя необходимо решить вязкие уравнения пограничного слоя (7.13) с $\rho_{0}=1$, дополненные условиями (7.16), (7.19) и (7.20). Одной из наиболее привлекательных особенностей модели трехпалубной структуры является ее универсальность, так как все специфические свойства модели содержатся в различных скейлингах.

Для решения парадокса Даламбера и “доказательства” справедливости условия Кутты-Жуковского (Вилля) необходимо ввести трехпалубную структуру вблизи задней кромки. Это продиктовано тем, что (асимптотическая) структура пограничного слоя Прандтля приводит к особенности на той стороне задней кромки, которая лежит ниже по течению. Это в свою очередь связано с тем, что изменение в граничном условии, когда течение отрьвается от крыла и входит в спутньй след, является разрьвньм. Однако гипотеза Кутты-Жуковского (Вилля), согласно которой коэффициент подъемной силы в результате саморегулирования принимает значение, которое устраняет наиболее существенную особенность, справедливо только в пределе при бесконечны $x$ числах Рейнольдса, и следует ожидать, что область, в которой течение жидкости вблизи задней кромки не удовлетворяет граничному условию, очень мала! Оказьвается, что ее размер пренебрежимо мал и имеет порядок $\mathrm{Re}^{-3 / 8}$, ипоэтому поправка подъемной силы имеет порядок $\mathrm{Re}^{-1 / 8}$. Однако, если угол атаки больше некоторой величины, критическое значение которой имеет порядок $\operatorname{Re}^{-1 / 16}$, происходит отрьв, которьй заполняет всю подветренную сторону профиля. По-видимому, часть жидкости, оторвавшейся вблизи задней кромки, может повторно присоединиться на достаточно близком расстоянии от задней кромки. Для профилей с толшиной, когда возможно появление гистерезиса, модель течения является еше более сложной в том, 
что касается зависимости оторвавшегося потока от малого параметра толщины. Довольно сомнительна возможность прояснения структуры течения вблизи угловой задней кромки путем полномасштабного моделирования (несжимаемых) уравнений Навье при больших числах Рейнольдса [65; с. 8]. Для того чтобы не было отрыва, угол задней кромки должен быть достаточно малым, а именно, порядка $\operatorname{Re}^{-1 / 4}$. Вопрос о рождении вихря, который иногда обсуждается в литературе, является бессмысленным в случае модели идеальной жидкости: если задняя кромка имеет конечный угол, то течение отрывается, и вихревая пелена вблизи задней кромки не образуется; с другой стороны, если задняя кромка такова, что отрыва не происходит, то модель идеальной жидкости соответствует бесконечному числу Рейнольдса при нулевом угле задней кромки, и в этом случае всегда образуется вихревая пелена в касательном направлении к сторонам кромки. По-видимому, трехмерньй анализ Манглера и Смита [105] не учитывает это обстоятельство, к которому трехмерность не имеет никакого отношения, так как вариации течения вблизи кромки контролируются двумернылми әффектами. В любом случае задача остается, в основном, открытой даже при условии, что игнорируются как нестационарность, так и турбулентность.

Необходимо также отметить, что разумное объяснение отрыва с помощью трехпалубной структуры при дозвуковом течении вне пограничного слоя было обнаружено Сьчевым [100] и дополнено численным кодом Смита [106].

В статье Гиро и Зейтуняна [107] получен критерий для нахождении линии отрьва вихревой пелены от гладкого тела. Этот критерий обобщает (в случае стационарного сжимаемого течения идеального газа с постоянной удельной теплоемкостью) критерий Смита (1978 г.) для несжимаемого безвихревого течения. Указанный критерий получен как следствие согласованности анализа идеальной жидкости и моделирования ламинарного пограничного слоя вблизи линии отрыва (в стиле Сычева) при помощи трехпалубной структуры. При этом предполагается, что стенка либо является адиабатической, либо имеет постоянную температуру, а компонента скорости стенки, перпендикулярная к линии отрыва, является дозвуковой.

В заключение, следуя Смиту [108], отметим, что трехпалубная структура является интерактивной моделью, причем эта интерактивная структура применима к достаточно большому числу ситуаций, в случае которых иерархическая структура не работает (см., например, книгу [109]).

Фильтрация и нестационарная корректировка. Когда $M \rightarrow 0$ (течения с мальм числом Маха), основные аппроксимации и асимптотические модели строятся с целью отделения быстрых акустических волн от решений полных сжимаемых уравнений Навье-Стокса (или Эйлера), так как такие волны не представляют интереса для медленных аэродинамических и различных атмосферных и океанических движений.

Когда $\operatorname{Re} \rightarrow \infty$ (течения с большим числом Рейнольдса), из (несжимаемых) уравнений Навье вьводятся уравнения пограничного слоя Прандтля (см., например, $(7.13))$, и согласно (7.13c) в случае нестационарного течения в предельном уравнении сохранения импульса для вертикальной компоненты скорости слагаемое $\partial v / \partial t$ пропадает! В случае малых чисел Рейнольдса $(\operatorname{Re} \rightarrow 0)$ в предельных (стационарных) уравнениях Стокса и Осеена нестационарный член также отсутствует!

Во всех обсуждавшихся выше случаях необходимо уточнить начальные условия, число которых меньше, чем для полных, “точных" уравнений Навье-Стокса (или 
Эйлера). Это связано с тем, что в результате предельного перехода, приводящего к асимптотической модели, некоторые производные по времени пропадают. Вследствие этого возникает вопрос: какие начальные условия можно налагать и как они связаны с начальными условиями для полных, точных уравнений Навье-Стокса (или Эйлера)?

Отметим, что уравнения Навье-Стокса, вообще говоря, не согласованы с оценками основных порядков величин, вытекающими из асимптотической модели. Для того чтобы начальные данные стали согласованными друг с другом в том, что касается их порядков, требуется физический процесс эволюции по времени. Такой процесс называется нестационарной корректировкой начального множества данньх, связанной с асимптотической структурой рассматриваемой модели. Процесс корректиров$\kappa u$, который наряду с метеорологией встречается также во многих областях гидромеханики, является коротким во временном масштабе изучаемой асимптотической модели, и он приводит, в асимптотическом смысле, к значениям начальных данных, которые подходят для данной модели.

При рассмотрении основных модельных уравнений (полученных асимптотически из полных, “точных" уравнений Навье-Стокса) необходимо объяснить процесс корректировки в случае уравнений Навье, уравнений пограничного слоя Прандтля, стационарных уравнений Стокса и Осеена, уравнений Буссинеска и неупругих уравнений.

Многие проблемы корректировки, возникающие в гидромеханике, связаны с потерей начальных условий в результате потери производных по времени при предельных переходах, приводящих к упрощенному множеству модельных уравнений. Здесь мы обсудим только наиболее известную проблему, которая изучалась многими специалистами по гидродинамике. Она состоит в потере начальных условий для полной функции распределения при переходе от уравнения Больцмана к уравнениям Навье-Стокса путем устремления к нулю отношения среднего свободного пробега и макроскопического масштаба длины $(L)$ (называемого числом Кнудсена); см., например, книгу Серсигнани $[110 ;$ гл. V, 55$]$. Насколько нам известно, эти задачи решены путем скейлинга временной и, возможно, некоторых пространственных переменных, что приводит к так называемой задаче начального слоя.

По сушеству, в зависимости от задачи могут быть два типа асимптотического поведения, когда время, полученное в результате скейлинга, стремится к бесконечности. А именно, либо наблюдается стремление к предельному стационарному состоянию, либо имеется незатухающее множество осцилляций (так обстоит дело, например, в случае инерционных волн в невязкой задаче о спинапе ${ }^{2}$ (разгоне) врашающейся жидкости; см., например, книгу Гринспена [111; раздел 24]).

Важно также отметить, что фильтрация акустических волн в полных нестационарных сжимаемых уравнениях Эйлера, когда $M \rightarrow 0$, меняет гиперболический характер уравнений Эйлера (см., например, [7]); например, предельньй переход в уравнениях Эйлера, когда

(7.21) $M \rightarrow 0$ и $B o \rightarrow 0$, причем $B o / M=B^{*}=O(1)$,

\footnotetext{
${ }^{2}$ Для жидкости, заключенной между двумя вращающимися концентрическими плоскостями, спинап определяется как переходный режим, возникаюший между двумя состояниями твердого вращения при увеличении угловой скорости. В произвольном замкнутом объеме спинап имеет аналогичньй смысл. Прим. перев.
} 
приводят к уравнениям Буссинеска, которые не являются гиперболическими (Вотак называемое число Буссинеска; см. [5]). Чтобы получить “правильные” начальные значения, необходимые для однозначного определения решения, для каждого из асимптотических модельных уравнений следует изучать (внутреннюю) начальную задачу о нестационарной корректировке в окрестности точки $t=0$ (при внешних временах порядка $O(1))$.

Отметим, наконец, что нестационарная корректировка аэродинамических (или метеорологических) полей является результатом образования, дисперсии и затухания быстрых инерционных волн.

Согласно МСАР начальные условия для предельных модельных уравнений представляют собой условия согласования для двух асимптотических представлений - основного (при фиксированном $t$ ) и локального (вблизи точки $t=0$ ).

В заключение можно сказать, что целью задачи нестационарной корректировки является выяснение вопроса о том, как мнохсество начальных данных, ассоциированное с определенной (точной) системой уравнений, связано с множеством начальных данных для более простых, приближенных модельных уравнений, которые рассматриваются как существенное вырождение исходной системы (точных) уравнений. Для решения этой задачи необходимо ввести начальный слой в окрестности точки $t=0$ путем скейлинга времени и введения новых неизвестных, не фигурирующих в начальной постановке.

Существование, единственность, устойчивость и регулярность решений: новейшие результаты. В приводимый ниже обзор мы включили результаты, которые, на наш взгляд, содержат полезную дополнительную информацию, однако ссылки не являются исчерпывающими и на их основе нельзя оценивать вклад разных физиков и математиков в исследование рассматриваемых задач.

Прежде всего отметим недавнюю книгу Лионса [20]. Этот двухтомник представляет собой единую и строгую монографию о различных математических аспектах гидромеханических моделей. В первом томе основной упор делается на математическом исследовании несжимаемых моделей (уравнения Навье, включая неоднородный случай, и уравнения Эйлера). С полными доказательствами излагаются некоторые известные и новые результаты о существовании и регулярности решений. Обсуждение содержит много интересных замечаний и интуитивных объяснений. В книге шшироко используются современная техника и методы, а также формулируется ряд нерешенных проблем. Второй том посвяшен в основном новым результатам для сжимаемых моделей (изэнтропические уравнения Навье-Стокса).

Касаясь (невязких) течений Эйлера, отметим наиболее свежий математический результат Оказава [112]. Целью этой статьи является доказательство локального существования и единственности классического решения уравнений Эйлера для несжимаемой жидкости в ограниченной области пространства $\mathbb{R}^{m}$. Доказательство основано на недавно установленной теореме об абстрактных квазилинейных эволюционных уравнениях (см. [113]). Для применения этой теоремы автор существенно использует результат Ж.-М. Гидалья [114] о максимальной гладкости. Относительно новый результат о глобальном существовании и единственности течения Эйлера в $\mathbb{R}^{2}$ для соответствуюших, возможно расходящихся на бесконечности начальных полей скоростей установлен в [115]. Однако анализ автора не включает физически интересньй 
случай однородного течения, для которого начальная завихренность (при $t=0$ ) принадлежит лишь $L_{\infty}$. В [116] доказьвается существование и компактность (устойчивость) энтропийных решений гиперболических систем законов сохранения, соответствующих изэнтропической газовой динамике, когда давление и плотность связаны $\gamma$-законом с $\gamma>1$. Авторы существенно обобщают и упрощают результаты Ди Перна (см. $[117],[118])$ и дают полное доказательство сушествования. Методы этой работы основаны на компенсированной компактности и кинетической формулировке систем законов сохранения. Работа Бренье [119] основана на результатах самого автора. Эта обзорная статья посвяшена некоторым аспектам так назьваемого современного лагранжева подхода в задачах идеальной гидродинамики, предложенного В.И. Арнольдом. Такой подход основан на представлении течения жидкости в компактной области в виде кривой в группе сохраняюших время диффеоморфизмов области. В заметке [120] дается простое доказательство гипотезы Л. Онсагера [121] о сохранении энергии для слабых решений трехмерных несжимаемых уравнений Эйлера. В работе [122] изучается вопрос о существовании стационарного напорного течения баротропной жидкости в плоскопараллельном слое с верхней поверхностью и постоянным гравитационным полем. Автор рассматривает такое течение как нестационарное в системе координат, где оно является напорным (строго или асимптотически). В работе Блохина и Биркина [123] изучается задача о глобальной разрешимости так называемой нестационарной модели сверхзвукового течения вблизи бесконечного кругового конуса в одномерном случае. Обсуждается справедливость метода стационаризации (стабилизации), который широко используется для численного решения задач газовой динамики. Корректность задачи Коши для коротковолновых-длинноволновых резонансных уравнений (предложенных Д. Дж. Бенни [124]) рассматривается в [125].

Касаясь (вязких несжимаемых) течений Навье, прежде всего отметим работу Константина [126]. Это очень элегантный, полный и доступный обзор о несжимаемых уравнениях Навье-Стокса. Делая акцент на нерешенные проблемы, автор приводит ряд новых, интересных результатов (полученных им самим и другими авторами), касаюшихся задач со свободной гранищей, поведения решений в турбулентном режиме и при больших временах, уравнений Эйлера и образования особенностей. В статье Бен-Артци [127] установлен интересный результат о существовании и единственности решения для двумерных уравнений Навье при условии, что начальная завихренность принадлежит $L^{1}$. Доказывается также, что когда вязкость стремится к нулю, некоторая последовательность решений уравнений Навье сходится к решению уравнений Эйлера! Целью заметки Брезиса [128] является доказательство того, что одно техническое предположение в статье [127] (а именно, условие (1.19) о поведении на бесконечности) не является необходимым для единственности. Интересно отметить, что статья [127] дает новьй подход в теории сушествования и единственности решения для системы нестационарных уравнений Навье в $\mathbb{R}^{2}$, которая описывает движение вязкой несжимаемой жидкости на всей плоскости с начальньм условием

$$
\boldsymbol{u}(\boldsymbol{x}, 0)=\boldsymbol{u}_{0}(\boldsymbol{x})
$$

и следующим условием на поведение на бесконечности:

$$
\boldsymbol{u}(\boldsymbol{x}, t) \rightarrow 0, \text { когда }|\boldsymbol{x}| \rightarrow \infty \text {, при } 0 \leqslant t<\infty .
$$


Этот подход основан на совершенно элементарньх принципах сравнения для линейных параболических уравнений. На первом шаге строится гладкое классическое решение для очень узкого класса начальных данных. Для этого используется итеративный метод, основанный на решении уравнения типа "конвекции с диффузией". Затем по непрерьвности сушествование решения распространяется на достаточно широкий класс начальных функций $\boldsymbol{u}_{0}(\boldsymbol{x})$. Эти результаты можно также перенести на случай мерозначных начальных данных. Однако "корректность" (в смысле сильной непрерывной зависимости от начальных данных) при таком обобщении теряется! В [127] дается также краткий обзор литературы, касающейся двумерных уравнений Навье-Стокса и соответствуюших предельных (невязких) уравнений Эйлера (см. с. 334-335). В статье Галди и Шора [129] доказьвается следуюший результат. Пусть $\{\boldsymbol{u}=(u, v), p\}$ - (гладкое) решение двумерных стационарных уравнений Эйлера, соответствуюшее заданному векторному полю $f$ (объемная сила, взятая с обратньм знаком) с ограниченным носителем ( $\boldsymbol{f}$ достаточно быстро убывает на бесконечности), удовлетворяюшее условию

$$
\boldsymbol{u}(\boldsymbol{x}) \rightarrow \boldsymbol{u}_{\infty} \text { при }|\boldsymbol{x}| \rightarrow \infty,
$$

где $\boldsymbol{u}_{\infty}=(1,0)$ - заданный постоянный вектор, и имеющее конечный интеграл Дирихле

$$
\int_{\Omega}(\nabla \boldsymbol{u} \cdot \nabla \boldsymbol{u}) d \Omega \leqslant M,
$$

где $M$ зависит только от начальных данных. Если

$$
\int_{|\boldsymbol{x}|>r}|v(\boldsymbol{x})|^{s} d x<\infty
$$

для некоторых $s \in(1, \infty)$ и $r>0$, то $\{\boldsymbol{u}=(u, v), p\}$ - физически разумное $(\Phi \mathrm{P})$ решение.

Решение называется физически разумным, если

$$
\boldsymbol{u}(\boldsymbol{x})-\boldsymbol{u}_{\infty}=O\left(|\boldsymbol{x}|^{-1 / 4-\varepsilon}\right)
$$

при больших $|\boldsymbol{x}|$ и некотором $\varepsilon>0$. Теорема сушествования в классе ФР решений в общем контексте установлена Галди (см. [130]). Напомним, что $\Omega$ (область течения) - дополнение двумерной компактной области (задача о плоском стационарном течении вокруг препятствия). Ясно, что условие (7.24) само по себе не может обеспечить сходимость $\boldsymbol{u}(\boldsymbol{x})$ к постоянному вектору $\boldsymbol{u}^{\circ}$, когда $|\boldsymbol{x}|$ стремится к бесконечности. Более того, легко привести примеры соленоидальных векторных полей, которые удовлетворяют условию (7.24) и растут на бесконечности. Вопрос о совпадении $\boldsymbol{u}^{\circ}$ и $\boldsymbol{u}_{\infty}$ остается открытым. Амик показал [131], что любая (достаточно гладкая) функция $\boldsymbol{u}(\boldsymbol{x})$, удовлетворяюшая стационарньм уравнениям Навье с $\boldsymbol{f}=0$, краевому условию на границе препятствия $\partial \Omega(\boldsymbol{u}(\boldsymbol{x})=0$ при $\boldsymbol{x} \in \partial \Omega)$ и условию конечности интеграла Дирихле (7.24), непременно ограничена. Возникает вопрос: отражают ли эти решения основные особенности, следуюшие из физических соображений, при условии, что 
вьполнено (7.23)? Например, можно ожидать, что они удовлетворяют уравнению сохранения энергии и что при $\boldsymbol{u}_{\infty} \neq 0$ соответствующее течение представляет собой бесконечный спутный след в направлении $\boldsymbol{u}_{\infty}$. Эти свойства связаны с асимптотической структурой на бесконечности. Исходя из таких мотиваций, разные авторы ввели класс $\Phi$ Р решений, удовлетворяющих (7.26)! Разумеется, в трехмерном случае поведение поля скоростей $\Phi$ Р решения отличается от (7.26).

Отметим здесь две недавние книги Галди, касаюшиеся математической теории уравнений Навье: [132], линейные стационарные задачи, и [133], нелинейные стационарные задачи. В статье Аманна [134] рассматривается движение вязкой несжимаемой жидкости в области $\Omega$ с условием непроскальзьвания на гранище $\partial \Omega$ и заданным начальным полем скоростей $\boldsymbol{u}_{0}(\boldsymbol{x})$. Тензор напряжений для несжимаемой вязкой жидкости определяется с помошью уравнения состояния как гладкая функция от линейного тензора скорости изменения деформаций. Жидкость назьвается ньютоновской, если эта функция линейна, и неньютоновской в противном случае. Если векторные поля плотности объемной силы и начальной скорости равны нулю, т.е. $\boldsymbol{f}=0$ и $\boldsymbol{u}_{0}=0$, то система уравнений, управляюшая движением, имеет тривиальное нулевое решение, соответствуюшее состоянию покоя жидкости, находящейся в ограниченной области $\Omega$ в пространстве $\mathbb{R}^{3}$. Согласно [134] решение устойчиво относительно малых возмушений функций $\boldsymbol{f}=0$ и $\boldsymbol{u}_{0}=0$, причем возмушенное решение әкспоненииально yбъвает $\kappa$ нулю, если этим свойством обладает возмущающая объемная сила.

По-видимому, результат об экспоненциальном убьвании возмушенного решения при наличии внешнего силового поля является новым даже в случае классических уравнений Навье (для ньютоновской жидкости). По поводу свойств убывания сильных решений двумерных нестационарных уравнений Навье см. статью Козоно и Огава [135]. Касаясь регулярности решений стационарных уравнений Навье в ограниченной области, отметим недавнюю статью Фрезе и Ружичка [136]. Работа Тани и Танака [137] посвящена сушествованию при больших временах поверхностных волн для несжимаемой вязкой жидкости с натяжением или без натяжения. Эта задача изучалась многими авторами (см., например, библиограффию в [137; с. 305-306]). В более свежей работе Тани [138] установлено существование решения в малом в случае трехмерных уравнений Навье для несжимаемой жидкости со свободной границей. Аналог квазидинамической системы Елизарова-Четверушкина [139] в случае вязкой несжимаемой жидкости построен в статье [140] путем формального обобщения. В этой работе получено уравнение баланса кинетической энергии для указанной математической модели и доказана теорема об убывании по времени полной кинетической энергии жидкости. Кроме того, показано, что решения системы Навье, описьвающие течения Куәтта и Пуазейля, являются точными решениями новой системы. Квазигидродинамические уравнения можно интерпретировать как регуляризацию уравнений Навье, необходимую для построения диссипативно устойчивых численных алгоритмов. Известно также, что для соответствуюшим образом регуляризованных гидродинамических уравнений установлено сушествование решения.

По поводу (вязких несэимаемых) течений Навъе-Стокса отметим недавнюю, очень содержательную статью Новотни и Падула [141]. В этой работе рассмотрено трехмерное стационарное течение, равномерное на бесконечности, для вязкой сжмимаемой жидкости, прошедшей через фиксированное препятствие. Авторами решены фундаментальные вопросы о существовании, единственности и асимптотике устано- 
вившегося течения вязкого идеального газа в тепловом равновесии. (На самом деле рассмотрено только изотермическое движение, для которого установлены существование и единственность решений в $L^{p}$-пространствах с $p>3$ и исследована гладкость, а также убьвание на бесконечности.) Эти фундаментальные проблемы изучались многими авторами. Сотни статей посвящены приближенным вычислениям, однако вопрос корректности с математической строгостью рассматривался лишь немногими авторами. В частности, для потенциального течения невязкой жидкости эти вопросы изучались в середине столетия (см., например, библиографию в книге Серрина [3]). Такое сильное ограничение на течение можно налагать ввиду того, что на большом расстоянии от препятствия ожидаемая завихренность течения близка нулю ( для вязкой несжимаемой жидкости можно доказать, что скорость убьвания является экспоненциальной). Следует отметить, что для течений в ограниченной области ряд важных результатов в математической теории вязких стационарных течений сжимаемой жидкости был получен за последние несколько лет (см. [142]-[145]). В случае неограниченных областей отметим результат о существовании (полученньй в [141]) для внешней области при условии, что скорость на бесконечности $\boldsymbol{u}_{\infty}$ равна нулю, а также результат Падула [146] для всего пространства $\mathbb{R}^{3}$ при достаточно малом $\boldsymbol{u}_{\infty}$.

Отметим, что системы, рассмотренные в [141], имеют для сжимаемой жидкости такую же важность, как и уравнения Стокса и Осеена для несжимаемой жидкости. С физической точки зрения такие уравнения пригодны для линейного приближения полной системы, когда конвективный член (типа Стокса) или его часть (типа Осеена) игнорируются только в уравнении сохранения количества движения. Это означает, что инерциальная составляющая много меньше члена, отвечающего за трение (медленное движение). Тем не менее доказательство можно применить к теплопроводящей жидкости и рассмотреть малое ненулевое граничное условие на $\partial \Omega(\Omega$ - внешность ограниченной области). Зависимость решений уравнений движения сжимаемой вязкой жидкости от числа Маха $M$ и коэффициентов вязкости, когда $M \rightarrow 0$ (слабо сжимаемая жидкость или течения с малым числом Маха), является очень интересной, но трудной задачей! В недавней статье Бейрао да Вейга [147] этот сингулярный предел рассматривается с математической точки зрения. В [147; с. 326-327] читатель может найти разные интересные ссылки, касаюшиеся сингулярных пределов в динамике сжимаемой жидкости. В [148] гидродинамический подход применяется для изучения течений с мальм числом Маха асимптотическими методами.

В выгислительной гидродинамике много дискуссий ведется о краевых условиях, которые следует налагать на удаленных границах. Такие границы возникают при срезании неограниченных областей (для сведения к задаче в ограниченной области). Для (сжимаемых) уравнений Навье-Стокса часто применяемые краевые условия были выведены из уравнений Эйлера, т.е. вязкие члены в уравнениях Навье-Стокса были игнорированы! Такой метод приводит к затруднительной ситуации, когда уравнения Навье-Стокса с налагаемымиграничными условиями представляют собой некорректную задачу, тогда как вычисления часто дают хорошие результаты. В статье Норстрёма [149] получены граничные условия, которые корректны при рассмотрении уравнений Навье-Стокса и полезны при вычислениях. Упомянутая статья содержит интересное обсуждение теории корректных граничных условий. Представлены численные результаты, подтверждающие полезность предложенных граничным условий. 
Устойчивость течений Навье во внешних областях изучается в статье Козоно и Огава [150]. Имеется по крайней мере два метода для исследования устойчивости стационарного течения несжимаемой вязкой жидкости. Первьй из них, энергетический метод, основан на изучении уравнений Эйлера-Лагранжа, а другой, метод линеаризованной устойчивости, - на исследовании спектра линейной пространственной части уравнений для возмущения. Первьй метод дает достаточньй критерий для энергетической устойчивости вне зависимости от величины начальных данных, тогда как второй приводит лишш к условию устойчивости стационарного течения при малых возмущениях. В конкретных случаях требуемую малость возмущения трудно контролировать [151]. Допустимые возмущения могут быть настолько малы, что с физической точки зрения возмущения конечной амплитуды недопустимы; такие возмушения называются инфинитезимальным.м. Важность второго метода состоит в том, что он дает достаточное условие неустойчивости (см., например, [152]). В статье фон Валя [151] обсуждается вопрос о совпадении достаточного условия устойчивости, полученного энергетическим методом и являющегося по существу критерием неустойчивости, и достаточного условия неустойчивости, установленного методом линеаризованной устойчивости.

Отметим, что имеется также более новьй, третий метод исследования проблемы устойчивости, который применим, в частности, к возмушениям неподвижного состояния. Исчерпывающий обзор этих вопросов (см. [151]) можно найти в работе Галди и Педула [153]. В [151] рассмотрены примеры плоскопараллельного течения вязкой жидкости с несимметричным профилем в бесконечном врашаюшемся слое и влияния вращения на конвекцию. В [154] с помощью преобразования Фурье-Лапласа изучается начально-краевая задача устойчивости для двумерного возмущения пограничного слоя Блазиуса (пограничный слой на плоской пластине, точнее, на полупространстве, помещенной кромкой в равномерное стационарное течение). Полученная неоднородная граничная задача для уравнения Орра-Зоммерфельда изучается аналитически.

Нелинейная неустойчивость связана, в основном, с непрерывным энергетическим каскадом, направленным к коротковолновой части спектра. Имеется много методов для избежания "нелинейной неустойчивости”, однако довольно специфический способ подавления этой неустойчивости состоит в оптимизации некоторых функиионалов. В статье Алексеева [155] при исследовании некоторых задач гидродинамики используется функционал погрешности для метода конечных разностей. Имеется обширная литература, касаюшаяся нелинейной устойчивости вязких ударных волн; разнообразные ссылки по этому поводу можно найти в статье Сзепесси и Ксин [156]. В этой работе изучается нелинейная устойчивость вязких законов сохранения при помощи анализа асимптотического поведения при больших временах ударной волны, возникаюшей в строго гиперболической системе; заметим, что под ударной волной в [156] понимается гладкая бегущая волна, удовлетворяющая условиям

$$
\frac{\partial u}{\partial x}+\frac{\partial f(u)}{\partial x}=\frac{\partial^{2} u}{\partial x^{2}}, \quad x \in \mathbb{R}^{1}, \quad t>0, \quad u \in \mathbb{R}^{m}, \quad u(\cdot, 0)=u^{\circ}(\cdot) .
$$

Отметим также важное наблюдение Т.-П. Лиу [157], согласно которому типичное возмущение вязкого ударного профиля порождает не только трансляционные, но и диффузионные волны. Отметим также, что ударные волны для сжсимаемых уравнений Навье-Стокса (в одномерном случае) устойчивы (см. [158]). 
Задача существования и линейной устойчивости для периодического волнового поезда Стокса в жидкости (воде) конечной глубины сформулирована в работе Бриджиса и Мильке [159] в терминах пространственно-временной гамильтоновой структуры задачи о волнах на воде (задача о волнах на воде обсуждается также в недавнем обзоре автора [90]). С гамильтоновой точки зрения доказана неустойчивость периодического волнового поезда Стокса. Строгий анализ дисперсионного соотношения доказьвает один результат, впервые отмеченный в середине шестидесятых годов (в работе Бенджамина, Файера [160]). Согласно этому результату гравитационньй волновой поезд достаточно малой амплитуды неустойчив при $F r \in\left(0, F^{\circ}\right)$, где $F^{\circ} \approx 0.8$, a $F r$ - число Фруда. Гамильтонова структура пространственного центрального многообразия, когда исходное уравнение имеет (конечномерную) гамильтонову структуру, была введена Мильке, а в [159] эта теория переносится на более общий случай, включающий результаты линейной устойчивости для волн с пространственным центральным многообразием. Недавно пространственная гамильтонова структура для задачи о стационарных волнах на воде изучалась в работе Гровса [161].

В теории гидродинамической устойчивости (краткий обзор нелинейной теории можно найти в [162]) течение Пуазейля, также как и задача Бенара-Рэлея, играет роль модели для аналитического и численного подходов. В обоих случаях в отличие от задачи Бенара о тепловой конвекции нейтральная кривая устойчивости может быть получена только численно. В работе Афендикова и Мильке [163] рассматриваются бифуркации течения Пуазейля между двумя параллельными пластинами и строятся трехмерные решения, имеюшие большую длину волны в направлении, перпендикулярном невозмушенному течению. Используя аналитические методы теории бифуркаций, такие как техника центрального многообразия, получены разные типы решений, сушествование которых трудно вывести другими методами, и установлена их устойчивость. Для этого численно находятся необходимые коэффициенты редуцированной задачи на центральном многообразии.

Касаясь результатов П.-Л. Лионса, важно отметить, что он доказал теорему $c y$ ществования для сжимаемых баротропных течений, которая является глобальной по времени и не предполагает малости начальных данных. С другой стороны, вопрос о единственности остается открытым (рассматриваются "слабые" решения). В некотором смысле Лионс установил (см., например, [164]) аналог результата Ж. Лере (или Э. Хопфа) о глобальном сушествовании для несжимаемых уравнений Навье. Из результата Валли о сжимаемых течениях вытекает глобальное существование ("сильного" и "гладкого") решения, но только при условии, что данные достаточно маль! В этом случае имеет место единственность.

Некоторые дополнительные результаты (декабрь 1996 года). Наряду с анализом уравнений волн на воде статья [165] содержит математическое обоснование модуляционного приближения. Доказывается, что полученный волновой пакет, оболочка которого управляется уравнением Шрёдингера с кубической нелинейностью (НУШ), является решением уравнений волн на воде. В работе Падула и Петунина [166] во внешней области рассматривается вязкая несжимаемая неоднородная теплопроводящая диффузионная жидкость в приближении Обербека-Буссинеска. Авторы строят глобальное регулярное решение с конечной кинетической энергией и находят скорость убывания его $L^{2}$-нормы. В статье [167] заслуживают внимания следуюшие результаты: новый вывод стационарного неоднородного уравнения Кортеве- 
га-де Фриза (СНКдФ), доказательство теоремы существования положительных решений СНКдФ и анализ ошибок с помошью сравнения. Асимптотическое поведение при $t \rightarrow \infty$ решений задачи Коши для уравнений, описываюших динамику теплопроводящей жидкости, рассмотрено в [168]. Статья [169] касается задачи, в которой уравнения Навье дополнены уравнением теплопроводности. В работе [170] доказьвается корректность (в смысле Адамара) характеристической симметрической гиперболической системы. Наконец, в [171] изучается устойчивость слабых волн разрежения системы законов сохранения, описьвающей одномерные вязкие среды со строго гиперболическими функциями тока.

\section{§ 8. Подход конечномерных динамических систем в задачах турбулентности}

Во многих случаях течение жидкости можно аппроксимировать конечномерной динамической системой. Такое приближение возможно, например, в стадии рождения турбулентности, когда имеется лишш конечное число возбужденных степеней свободы движения. На практике изучение так называемого "хаоса" проводится, в основном, методами конечномерных динамических систем (малой размерности), тогда как "турбулентность" связана с движением жидкости с бесконечным числом степеней свободы. Аппроксимация жидкости в терминах модельных конечномерных систем - мошное средство анализа, благодаря которому недавнее развитие теории хаоса позволило изучить фундаментальные механизмы турбулентности. С другой стороны, общепризнанно, что турбулентность в развитой стадии имеет сингулярную пространственно-временную структуру. Такое сингулярное поведение невозможно правильно описывать модельной системой конечной размерности. В этом смысле хаос в жидкости представляет собой лишь часть феномена турбулентности.

Несмотря на то, что хаос и турбулентность в жидкости - не одно и то же, образ турбулентного, беспорядочного движения служит для физиков указателем, напоминаюшем о задаче, которую следует изучить. Турбулентность в жидкости - крайне нерегулярное явление, не поддаюшееся точному прогнозу.

По сушеству, понятие хаоса связано с “чувствительной зависимостью от начальных данных". Формально “хаотическим” назьвается неслучайное сложное движение, в котором проявляется очень быстрьй рост ошибок, не позволяющий несмотря на детерминированность рассматриваемого процесса делать долгосрочные точные прогнозы.

Известно, что в некоторых системах с небольшим числом степеней свободы проявляется временной хаос. Рассмотрим, например, модель Лоренца [172], имеющую лиш три степени свободы. Это грубое усечение классической двумерной задачи Рэлея-Бенара о мелкой конвекции, которое содержит лишь по одному показателю Фурье в вертикальном и горизонтальном направлениях, так что движение никак не может рассматриваться как пространственно хаотическое! Тем не менее, численные расчеты показьвают, что временной спектр становится непрерьвным, когда число Рэлея пересекает некоторый пороговьй уровень - указатель существования временного хаoca.

Совершенно другой является задача о развитой турбулентности, которая, по сушеству, представляет собой пространственно-временной хаос: когда число Рей- 
нольдса стремится к бесконечности, возмущаются все пространственные и временные шкалы, включая инфинитезимальные. Такой хаос может возникнуть за конечное время и обладает универсальньми масштабньми характеристиками (например, степенной закон энергетического спектра). Универсальные свойства развитой турбулентности связаны с перемежаемостью или, по-другому, неоднородностью малых масштабов. Однако переход к турбулентности (хаосу!) возможен через перемежаемость (путь к хаосу Помо-Маневилля). В гидродинамике часто различают (см., например, обзорную статью Хюера [173]) поведение открытых и замкнутых систем. С чисто кинематической точки зрения можно сказать, что течение является замкнутым, если частищы жидкости периодически возврашаются в рассматриваемую физическую область (конвекция Бенара в горизонтальном слое жидкости). Если же все частицы окончательно покидают область (как при плоском течении Пуазейля), то течение называется открытым.

В неустановившихся замкнутых течениях движение является абсолютно неустойчивым (конвекция Рэлея-Бенара или течение Тейлора-Куэтта) и может привести при определенных условиях к хаотическому движению на странном аттракторе малой размерности. Этого не происходит в случае открытых, конвективно неустойчив $ы x$ течений! Возникновение хаоса в конвективно неустойчивом течении обсуждается B [173].

В 1944 году Ландау [174], а затем в 1948 году Хопф [175] выдвинули теорию для объяснения происхождения турбулентности, согласно которой физические параметры, объясняющие феномен турбулентности, являются квазипериодическими функциями вида

$$
f\left(\omega_{1} t, \omega_{2} t, \ldots, \omega_{k} t\right)
$$

где функция $f$ периодична по каждой из переменных с периодом 1, a $\omega_{i}, 1 \leqslant i \leqslant k,-p a-$ ционально независимые числа. (Это означает, что если некоторая линейная комбинация чисел $\omega_{i}$ с рациональными коэффициентами обращается в нуль, то все коэффициенты равны нулю.) Квазипериодическое движение с большим числом независимых частот может быть очень сложным и напоминает поведение в турбулентном режиме!

Тем не менее основное возражение теории (сценария) Ландау-Хопфа состоит в том, что квазипериодические движения не отражают важньй феномен, назьваемьй “чувствительной зависимостью от начальных данных" (ЧЗНД), который является существенной характеристикой турбулентности (хаоса). Это свойство ярко проявляется в том, что два течения, почти идентичные в начальный момент времени, сильно отличаются впоследствии, что связано с феноменом “әкспоненциальной расходимости".

Почти через двадцать лет после создания теории Ландау Лоренц [172] предпринял попытку приближенного описания конвективной неустойчивости Рэлея-Бенара в случае течений в атмосфере. Если температура достаточно высока, то имеет место беспорядочно турбулентная конвекция, причем временная эволюция такой турбулентности связана с появлением в фазовом пространстве (странного) аттрактора Лоренца, возникающего из ассоциированной системы Галеркина. Приводимая ниже иллюстрация такого аттрактора взята из работы ${ }^{3}$ О.Э. Лэнфорда (университет Беркли).

\footnotetext{
${ }^{3}$ См. также статью О. Лэнфорда "Изображение аттрактора Лоренца, полученное с помощью компьютера" в [176; с. 73-74]. Прим. перев.
} 
Динамическая система Лоренца, содержащая три уравнения, записывается в виде

$$
\frac{d X}{d t}=-10 X+10 Y, \quad \frac{d Y}{d t}=-X Z+28 X-Y, \quad \frac{d Z}{d t}=X Y-\frac{8}{3} Z .
$$

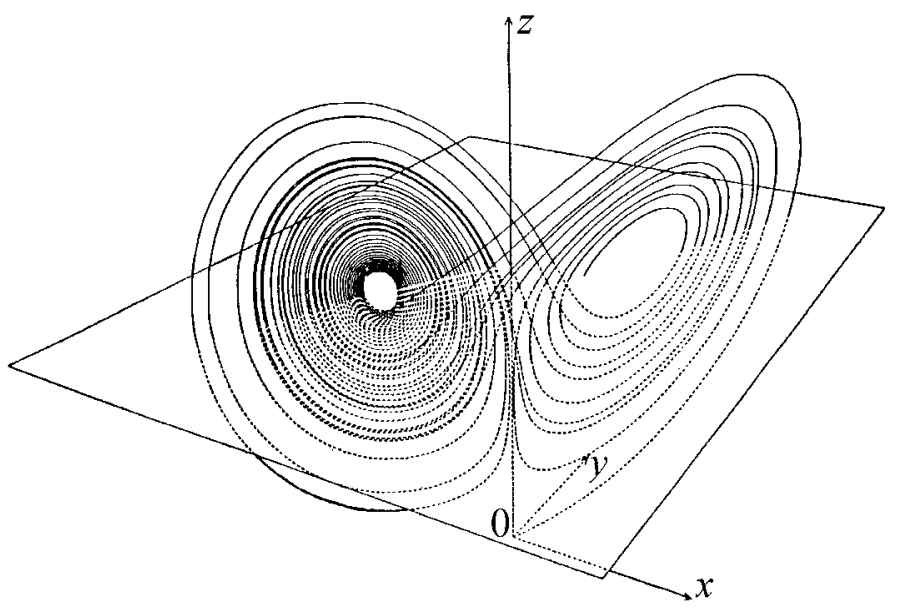

Рис. 2. Странный аттрактор Лоренца

Для уравнений Лоренца (8.1) Лэнфорд рассматривал решение, выходяшее из начала координат ( $X=0, Y=0, Z=0)$ в момент времени $t=0$. На самом деле при численном моделировании задавалось не “в точности” нулевое начальное условие, а “достаточно близкое" к нему. Это решение совершает один виток справа, затем несколько витков слева, потом опять справа и т. д., причем совершенно случайным образом. На рисунке можно проследить до пятидесяти витков решения, причем часть траектории, лежащая ниже уровня $Z=27$, изображена пунктиром. Если при $t=0$ вместо $(0,0,0)$ выбрана другая, но очень близкая начальная точка, то соответствуюшее решение системы (8.1) будет сильно отклоняться от исходного (экспоненциальная расходимость), причем число витков слева или справа также будет меняться из-за ЧЗНД. На основе этого явления Лоренц строго обосновал невозможность более или менее долгосрочного прогноза погоды, причем под долгосрочностью понимается промежуток времени более одной недели!

К сожалению, в то время специалисты по гидромеханике даже не подозревали о сушествовании связи между результатами Лоренца и их собственными исследованиями по проблеме турбулентности. Именно поэтому работа Лоренца [172] осталась незамеченной среди математиков и физиков.

Выражение “странный аттрактор" (аттрактор Лоренща попадает в эту категорию) было введено в работе Рюэля и Такенса [176], см. также [177]. Именно понятие ЧЗНД лежит в основе теории Рюэля-Такенса. Оно связьвает турбулентность со странным аттрактором ("странность" связана с ЧЗНД), возникающим в фазовом пространстве ассоциированной динамической системы. Этот аттрактор, являющийся абстрактным математическим объектом, образован из бесконечного числа тонких слоев или колец, растянутых и сложенных друг над другом. Следует иметь в виду, что в диссипативной динамической системе (описьваемой, например, вязкими несжимаемыми 
уравнениями Навье) траектории не покрывают все фазовое пространство, а стремятся к некоторой его части, назьваемой аттрактором, и все время остаются вблизи нее. Из-за этого структура аттрактора тесно связана с природой устанавливающегося предельного режима.

Поэтому в отличие от теории Ландау-Хопфа, для которой аттрактором, ассоциированным с турбулентньм режимом, является тор $T^{k}$ большой размерности $k$, в модели Рюэля-Такенса турбулентность описывается странньм аттрактором, который отражает феномен ЧЗНД, причем движение на странном аттракторе также является неустойчивым по Ляпунову и, следовательно, имеет чисто непрерывный энергетический спектр. Новая теория Рюэля-Такенса способствовала созданию огромного числа работ, посвященных изучению динамических систем, и дала толчок разнообразным экспериментам и вычислениям, касающимся перехода к “турбулентности” для различных физических систем (см., например, статью [179], содержашую многочисленные примеры странных аттракторов, возникаюших в задаче Бенара о глубокой конвекции).

Прошло десять лет после публикации работы Рюэля-Такенса, прежде чем было создано новое направление в теории турбулентности. В связи с этим в первую очередь отметим статью Фейгенбаума [180], хотя следует также подчеркнуть, что аналогичные результаты были получены в работе Трессера и Кулле [181]. В теории Фейгенбаума-Трессера-Куллепереход к турбулентности происходит благодаря явлению удвоения периодов, причем техника, известная как группа ренормализации (ГР), играет существенную роль. В настоящее время интенсивно изучается переход к хаосу от квазипериодических режимов (например, в конвективных течениях) или через перемежаемость (согласно модели Помо и Манневилля [182] перехода к хаосу), причем в последнем случае возникают турбулентные “следы” большой амплитуды. Касаясь перехода от периодического режима к хаотическому, отметим, что существуют различные сценарии; краткое обсуждение можно найти в работе Свинни [183].

Важно также заметить, что понятие хаоса, которое возникло в результате изучения динамических систем, связано с фрактальными размерностями и фрактальной геометрией. В теории динамических систем притягиваюшее множество, обладающее фрактальными свойствами, называется странным аттрактором! Математически фрактал определяется как “множество, для которого размерность Безиковича-Хаусдорфа строго больше топологической размерности”.

Аттракторы суть геометрические объекты, характеризующие поведение системы в фазовом пространстве (пространстве состояний) при больших временах. Грубо говоря, аттрактор это множество, к которому стабилизируется или стремится динамика. Простейшим примером аттрактора является неподвижная точка, представляющаяся собой состояние равновесия или, по-другому, стационарное решение динамической системы. Неподвижная точка в фазовом пространстве может притягивать (устойчивая неподвижная точка) или отталкивать (неустойчивая неподвижная точка) проходящие вблизи нее траектории. Поэтому при исследовании динамических систем полезно определить все неподвижные точки и изучить их устойчивость. Это в свою очередь мотивирует изучение бифуркаций, которые могут влиять на устойчивость стационарных точек. Более сложным аттрактором является предельный иикл-замкнутая петля в фазовом пространстве. Предельный цикл описывает устойчивые осцилляции (периодические решения). Если при рассмотрении устойчивого предельного 
цикла в трехмерном фазовом пространстве ввести двумерную поверхность Пуанка$p e$ (поверхность сечений), то траектория (или орбита) будет многократно пересекать эту поверхность в одной и той же точке. Наконец, торообразный аттрактор возникает в случае более сложных осциллящий (квазипериодическое движение). Поведение динамической системы, аттрактор которой принадлежит одному из указанных трех типов, допускает сколь угодно точное прогнозирование. С другой стороны, хаотические аттракторы соответствуют непредсказуемым движениям и имеют более сложную геометрическую структуру.

Ключ к пониманию хаотического движения дают простые операции растяжения и свертывания, которые происходят в фазовом пространстве. Прежде всего заметим, что экспоненциальная расходимость является локальным свойством: так как аттракторы ограничены, то две орбиты хаотического аттрактора не могут экспоненциально отталкиваться все время. Следовательно, аттрактор должен наматьваться сам на себя. Несмотря на то, что орбиты расходятся и расстояние между ними увеличивается, время от времени они подходят близко друг к другу. Траектории, лежащие на хаотическом аттракторе, перемешиваются подобно колоде карт в руках сдающего! Случайность хаотических орбит является результатом перемешивания. Процессы растяжения и свертьвания повторяются многократно, порождая бесконечное число вложенных складок. Другими словами, хаотический (странньй) аттрактор является фракталом - объектом, при увеличении которого обнаруживаются все больше особенностей (“другой взгляд” на странные аттракторы содержится в обзорной статье Гукенхаймера [184]).

Эксперименты, разработанные для изучения странных (хаотических) аттракторов в теории турбулентности, проводились, в основном, над проводяшими слоями (системы Рэлея-Бенара) и течениями между вращаюшимися цилиндрами (эксперимент Куэтта-Тейлора).

Метод Галеркина. Рассмотрим теперь нестационарную задачу для уравнений Навье в области $\Omega_{T}=\Omega \times[0, T]$ :

$$
\begin{gathered}
\frac{\partial \boldsymbol{V}}{\partial t}-\nu^{\circ} \Delta \boldsymbol{V}+\boldsymbol{V} \cdot \nabla \boldsymbol{V}=-\nabla p+\boldsymbol{f} \\
\nabla \cdot \boldsymbol{V}=0 \\
\left.\boldsymbol{V}\right|_{\partial \Omega}=0,\left.\quad \boldsymbol{V}\right|_{t=0}=\boldsymbol{V}^{\circ}
\end{gathered}
$$

где $\boldsymbol{f}(\boldsymbol{x}, t)$ - заданное векторное поле внешних сил, а $(\boldsymbol{V}, p)$ - неизвестные функции (вектор скорости и давление). Мы будем предполагать, что заданное векторное поле $\boldsymbol{V}^{\circ}(\boldsymbol{x})$ является соленоидальным и удовлетворяет граничному условию $\left.\boldsymbol{V}^{\circ}\right|_{\partial \Omega}=0$.

Пусть система $\left\{\varphi^{k}(\boldsymbol{x})\right\}$ ортонормированных в $L_{2}(\Omega)$ функций полна в пространстве $H(\Omega)$. Предположим, что функция $\varphi^{1}$ совпадает с заданным начальным условием для искомого решения: $\varphi^{1}(x)=\boldsymbol{V}^{\circ}(\boldsymbol{x})$. Приблизим решение с помошью функций $\boldsymbol{V}^{n}$ вида

$$
\boldsymbol{V}^{n}=\sum_{l=1}^{n} C_{l n}(t) \varphi^{l}(\boldsymbol{x})
$$


Коэффициенты $C_{l n}(t)$ определяются из соотношений

$$
\begin{gathered}
C_{l n}(0)=\delta_{l 1} \\
\left\langle\frac{\partial \boldsymbol{V}^{n}}{\partial t}+\boldsymbol{V}^{n} \cdot \nabla \boldsymbol{V}^{n}-\boldsymbol{f}, \varphi^{l}\right\rangle+\nu^{\circ}\left\langle\nabla \boldsymbol{V}^{n}, \nabla \varphi^{l}\right\rangle=0
\end{gathered}
$$

где $l=1,2, \ldots, n$, а через $\langle\cdot, \cdot\rangle$ обозначается скалярное произведение в $L_{2}(\Omega)$. Это и есть известный метод Галеркина. Ввиду ортонормированности функций $\varphi^{l}(x)$ в $L_{2}(\Omega)$ уравнения $(8.4 \mathrm{~b})$ образуют систему обыкновенных дифференциальных уравнений относительно $C_{l n}(t)$ :

$$
\frac{d C_{l n}(t)}{d t}=\nu^{\circ} \sum_{k=1}^{n} a_{k l} C_{k n}+\sum_{k, p=1}^{n} a_{k p l} C_{k n} C_{p n}+f_{l}, \quad l=1, \ldots, n
$$

где $a_{k l}$ и $a_{k p l}$ - числовые коэффициенты ${ }^{4}$, a $f_{l}=\left\langle\boldsymbol{f}, \varphi^{l}\right\rangle$. Правые части уравнений (8.5) являются аналитическими функциями по $C_{l n}$. Поэтому сушествование единственного решения системы (8.4b) с начальными условиями (8.4a) на интервале определения правой части будет установлено, если мы покажем, что решения $C_{l n}(t)$ ограничены на этом интервале. Отметим, что ограниченность является следствием наложенных условий [185]:

$$
\boldsymbol{V}^{\circ} \in W_{2}^{2}(\Omega), \quad \int_{0}^{T^{*}}\left[\|f\|+\left\|\frac{\partial f}{\partial t}\right\|\right] d t<\infty,
$$

где $T^{*}$ - положительная константа.

Уравнения (8.5) представляют собой конечномерную динамическую систему размерности $n$ относительно амплитудных функций $C_{l n}(t), l=1, \ldots, n$.

Впервые странный аттрактор, возникающий в задачах гидродинамики, был обнаружен Лоренцем [172]. Уравнения Буссинеска, описываюшие тепловую мелкую конвекцию Рэлея-Бенара для горизонтального слоя подогреваемой снизу жидкости, Лоренц разложил в тройной ряд Фурье по пространственным переменным (метод Галеркина), выписал обыкновенные дифференциальные уравнения для коэффициентов Фурье, зависящих от времени, а затем рассмотрел усеченную систему, состоящую из трех уравнений.

Если обозначить коэффициенты Фурье, фигурируюшие в этих трех уравнениях, через $X(t), Y(t)$ и $Z(t)$, то система Лоренца примет вид

$$
\begin{aligned}
& \frac{d X}{d t}=-\sigma X+\sigma Y, \\
& \frac{d Y}{d t}=r X-Y-X Z, \\
& \frac{d Z}{d t}=-b Z+X Y .
\end{aligned}
$$

\footnotetext{
${ }^{4}$ Легко видеть, что эти коэффициенты имеют вид $a_{k l}=\left\langle\nabla \varphi_{k}, \nabla \varphi_{l}\right\rangle, a_{k p l}=\left\langle\varphi_{k} \cdot \nabla \varphi_{p}, \varphi_{l}\right\rangle$. Прим. перев.
} 
Константа $r>0$ пропорциональна числу Рэлея (отношение числа Рэлея к критическому числу) и представляет собой меру интенсивности подогрева (на самом деле, $r$ - управляющий параметр), $\sigma>0$ - число Прандтля, а $b>0$-геометрический коэффициент коробки.

Бифуркации в диссипативных динамических системах. Рассмотрим динамическую систему общего вида с $N$ степенями свободы:

$$
\frac{d \boldsymbol{X}}{d t}=F(\boldsymbol{X} ; \lambda), \quad \boldsymbol{X}(t) \in \mathbb{R}^{N}
$$

Под динамической системой (ДС) часто понимают систему достаточно малой размерности $N$. Для диссипативной ДС объемы в фазовом пространстве сжимаются, а объектами изучения являются траектории и их аттракторы в фазовом пространстве (касаясь характерных неустойчивостей и нелинейной динамики, отметим работу Тюала [186]). Когда управляющий параметр $\lambda$ варьируется, эти аттракторы могут дестабилизироваться, и в фазовом портрете происходят топологические изменения - бифуркации. Описанная выше модель Лоренца (8.6) - первый пример такой диссипативной ДС.

Очевидно, что для заданной ДС точка $\boldsymbol{X}^{*}$ фазового пространства является неподвижной (точка равновесия), если $F\left(\boldsymbol{X}^{*} ; \lambda\right)=0$. Для исследования ее устойчивости необходимо изучить собственные значения матрицы размера $N \times N$, полученной в результате линеаризации уравнения в точке $\boldsymbol{X}^{*}$ :

$$
\begin{gathered}
\boldsymbol{X}=\boldsymbol{X}^{*}+\boldsymbol{U}, \\
\frac{\partial \boldsymbol{U}}{\partial t}=L^{*} \boldsymbol{U}+N(\boldsymbol{U}, \lambda),
\end{gathered}
$$

где $L^{*}=D F\left(\boldsymbol{X}^{*} ; \lambda\right)$ - производная функции $F\left(\boldsymbol{X}^{*} ; \lambda\right)$ в точке $\boldsymbol{X}^{*}$. Неподвижная точка устойчива, если все собственные значения (линейного) оператора $L^{*}$ имеют отрицательные вещественные части. Неустойчивость возникает тогда, когда одно действительное или два комплексно-сопряженные собственные значения (в обшем случае) пересекают мнимую ось. Топологическая особенность, ассоциированная с неустойчивостью, называется бифуркацией.

Когда мнимую ось пересекает вещественное собственное значение, типичной является седло-узловая бифуркация: две неподвижные точки, одна из которых является неустойчивой, а другая - устойчивой, соединяются при некотором критическом значении параметра. При этом число решений уравнения $F\left(\boldsymbol{X}^{*} ; \lambda\right)=0$ меняется, так как производная по $\boldsymbol{X}$ функции $F$ не обратима, и поэтому теорема о неявной функции не применима. Если рассматриваемое уравнение обладает некоторой симметрией (нетипичный случай), имеет место так называемая вильная бифуркация.

Для модели Лоренца (8.6) производная функции $F\left(\boldsymbol{X}^{*} ; \lambda\right)$ в точке $\boldsymbol{X}=(X, Y, Z)$ записывается в виде матрицы размера $3 \times 3$ :

$$
D F\left(\boldsymbol{X}^{*}, \lambda\right)=\left(\begin{array}{ccc}
-\sigma & \sigma & 0 \\
r-Z & -1 & -X \\
Y & X & -b
\end{array}\right)
$$


Решая характеристическое уравнение при $\boldsymbol{X}=0$, находим соответствуюшие собственные значения:

$$
(s+b)\left[s^{2}+(\sigma+1) s+\sigma(1-r)\right]=0 .
$$

Когда $r=1$, нулевое решение испытывает вильную бифуркацию. Уравнения инвариантны относительно преобразования

$$
X \Rightarrow-X, \quad Y \Rightarrow-Y, \quad Z \Rightarrow-Z
$$

При $r>1$ нулевое решение неустойчиво, а два симметричных решения

$$
\boldsymbol{X}^{+}(a, a, r-1) \text { и } \boldsymbol{X}^{-}(-a,-a, r-1),
$$

где $a=[b(r-1)]^{1 / 2}$, устойчивы. Эти новые неподвижные точки физически соответствуют конвективным врашениям (по часовой стрелке или против нее).

Характерной для комплексно-сопряженных собственных значений является бифуркаиия Хопфа, и в этом случае оператор $L^{*}$ остается обратимым. В результате бифуркации около неподвижной точки появляется охватывающая ее периодическая круговая траектория с возрастающим радиусом.

Нормальная форма вильной бифуркации. Вильная бифуркация возникает тогда, когда имеется вещественное собственное значение, пересекающее мнимую ось при некотором $\lambda=\lambda_{c}$, а уравнения обладают симметрией. Пусть $L=L_{c}$ - линеаризованный оператор в критической точке, $L \varphi=0, \varphi$ - маргинальная мода, а $L \varphi_{i}=s_{i} \varphi_{i}$, $\varphi_{i}$ - демпфированные моды.

Для любого $\lambda$ рассмотрим разложение $\boldsymbol{U}(t)$, согласованное с $L$ :

$$
\boldsymbol{U}(t)=A(t) \varphi+\sum B_{i}(t) \varphi_{i}
$$

Из уравнения $\partial \boldsymbol{U} / \partial t=L \boldsymbol{U}+N(\boldsymbol{U}, \lambda)$ находим

$$
\frac{d A}{d t}=\mu A+g(A, B), \quad \frac{d B_{i}}{d t}=s_{i} B_{i}+g_{i}(A, B),
$$

где $\mu$ - бифурцирующее собственное значение, эквивалентное $\left(\lambda-\lambda_{c}\right)$ вблизи $\lambda_{c}$. Характерное время эволюции для демпфированных мод имеет порядок единицы, тогда как для маргинальной моды этот порядок равен $(1 / \mu)$. Поэтому мы предполагаем, что функция $B$ адиабатически связана с эволюцией $A$, т.е. $B=h(A)$, или, более аккуратно, привлекая теорему о иентральном многообразии, $B=h(A)=$ $a A^{2}+b A^{3}+O\left(A^{4}\right)$. Теперь мы можем исключить $B$ из эволюционного уравнения для $A: d A / d t=\mu A+g(A, h(A))=: \mu A+f(A)$. Наконец, ввиду симметрии $A \Rightarrow-A$, приводящей к вильной бифуркации, асимптотическое разложение $f(A)$ начинается с кубического члена: $f(A)=-\alpha A^{3}+O\left(A^{5}\right)$.

Таким образом, мы получили главные члены нормальной формы вильной бифуркации:

$$
\frac{d A}{d t}=\mu A-\alpha A^{3}
$$


Это хорошо известное уравнение Ландау. В зависимости от знака $\alpha$ вильная бифуркация является суперкритической $(\alpha>0)$ или субкритической $(\alpha<0)$.

Уравнение Ландау для вильной бифуркации в модели Лоренца (8.6) соответствует значениям

$$
\mu=\frac{\sigma(r-1)}{\sigma+1}, \quad \alpha=\frac{\sigma}{b(\sigma+1)}>0
$$

Нормальная форма бифуркации Хопфа. Предположим, что комплексно-сопряженные собственные значения $i \omega$ и $-i \omega$ пересекают мнимую ось при $\lambda=\lambda_{c}$. Мы сохраним использованные вьше обозначения (см. [186]): $L=L_{c}$ - линеаризованный оператор в критической точке, $L \varphi=i \omega \varphi$ и $L \varphi^{*}=-i \omega \varphi^{*}, \varphi$ и $\varphi^{*}$ - маргинальные моды, а $L \varphi_{i}=s_{i} \varphi_{i}, \varphi_{i}$ - демпфированные моды. Для любого $\lambda$ рассмотрим разложение $\boldsymbol{U}(t)$, согласованное с $L$ :

$$
\boldsymbol{U}(t)=W(t) \varphi+W^{*}(t) \varphi^{*}+\sum B_{i}(t) \varphi_{i}
$$

Напомним, что решением линейной задачи $\partial \boldsymbol{U} / \partial t=L \boldsymbol{U}$ является семейство эллипсов:

$$
\boldsymbol{U}(t)=2 a \cos (\omega t-\gamma) \boldsymbol{V}_{r}-2 a \sin (\omega t-\gamma) \boldsymbol{V}_{i}, \quad \boldsymbol{V}=\boldsymbol{V}_{r}+i \boldsymbol{V}_{i}
$$

Нелинейная задача $\partial \boldsymbol{U} / \partial t=L \boldsymbol{U}+N(\boldsymbol{U}, \lambda)$ эквивалентна системе

$$
\begin{aligned}
\frac{d W}{d t} & =(\mu+i \omega) W+g\left(W, W^{*}, B\right), \\
\frac{d W^{*}}{d t} & =(\mu-i \omega) W^{*}+g^{*}\left(W, W^{*}, B\right), \\
\frac{d B_{i}}{d t} & =s_{i} B_{i}+g_{i}\left(W, W^{*}, B\right),
\end{aligned}
$$

где $\mu$ - вешественная часть бифурцирующих собственных значений, пропорциональная $\lambda-\lambda_{c}$. В этом случае разложение центрального многообразия имеет вид

$B=h\left(W, W^{*}\right)=a W^{2}+b W W^{*}+c W^{* 2}+d W^{3}+e W^{2} W^{*}+p W W^{* 2}+q W^{* 3}+O\left(|W|^{4}\right)$.

Мы можем теперь исключить $B$ из эволюционного уравнения для $W$ :

$$
\frac{d W}{d t}=(\mu+i \omega) W+g\left(W, W^{*}, h\left(W W^{*}\right)\right)=(\mu+i \omega) W+f\left(W, W^{*}\right) .
$$

Асимптотическое разложение функции $f\left(W, W^{*}\right)$ начинается с квадратичных членов, однако нелинейной заменой переменных (см. [186; с. 12]) мы можем исключить все коэффициенты асимптотического разложения функции $f\left(W, W^{*}\right)$ за исключением резонансных членов. Это приводит к нормальной форме бифуркации Хопфа, главная часть которой записывается в виде:

$$
\frac{d W}{d t}=(\mu+i \omega) W-\alpha|W|^{2} W
$$

где $W$ и $\alpha$ - комплексные числа. 


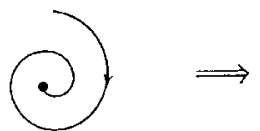

(a)

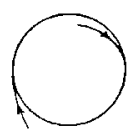

(b)

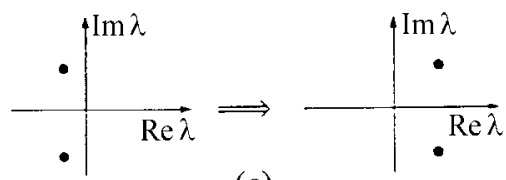

(c)

Рис. 3. Бифуркация Хопфа от неподвижной точки (a) к предельному циклу (b) и поведение собственных значений $\lambda(\mathrm{c})$

В зависимости от знака $\operatorname{Re} \alpha$, бифуркация является суперкритической $(\operatorname{Re} \alpha>0)$ или субкритической $(\operatorname{Re} \alpha<0)$ с $\mu=0$. Вопрос о том, является ли бифуркация Хопфа суперкритической или субкритической, нетривиален. Выгисление нормальной формы, ассоциированной с этой бифуркацией, приводит к ответу на этот вопрос. Отметим также, что вычисление (вывод) нормальной формы можно осуществить либо асимптотическими методами, включающими малый параметр (метод Пуанкаре-Линдштедта, метод многих масштабов и т. д.), либо прямым методом Крылова-Боголюбова-Митропольского (см., например, [186; с. 12-17]). Однако, используя технику типа Ляпунова-Шмидта, полученную из теории бифуркаций и теории возмущений, сопоставленной с методом многих масштабов, можно вывести (как и в [187]) систему уравнений, управляющую эволюцией амплитуды наиболее быстрорастуших мод линейной теории. Если имеется только одна растущая мода (и в этом случае происходит так назьваемая перестановка устойчивостей), то для соответствуюшей амплитуды снова получаем классическое уравнение Ландау (8.12). Эвристические рассуждения, обосновьвающие уравнение Ландау, можно найти в [188].

Бифуркация от периодической орбиты к инвариантному тору. В результате бифуркации, следуюшей за той, которая привела к замкнутой траектории и, следовательно, к периодическому движению, может появиться инвариантный (устойчивый) двумерный тор $T^{2}$. Однако возникновение инвариантного двумерного тора в результате бифуркации периодической устойчивой орбиты вовсе не означает, что существуют траектории, плотные на этом торе. В самом деле, в общем случае маловероятно, чтобы одна орбита была плотна на полученном двумерном торе и, следовательно, являлась квазипериодической функцией времени (ввиду теоремы Пейксото [19; т. II, с. 354]). Появление конечного числа периодических орбит и неподвижных точек характерно, тогда как квазипериодические движения не характерны. Возникновение инвариантного трехмерного тора при следуюшей бифуркации зависит от существования орбиты, плотной на двумерном торе, и поэтому бифуркация к трехмерному инвариантному тору маловероятна! Если периодическая орбита, лежащая на двумерном торе, совершает $n$ витков прежде чем замкнуться, то бифуркация является субгармонической с внезапным $n$-кратным увеличением периода при бифуркации. В случае эксперимента Бенара после появления двух основных частот энергетический спектр становится непрерывным (хаос). Уже после двух бифуркаций Хопфа регулярное движение ста- 
новится крайне неустойчивым из-за хаотического движения на странном аттракторе. Понятно, что хаотическое движение возможно только после двух бифуркаций Хопфа, когда траектория становится многомерной. Это следует из того, что двоякопериодическое движение соответствует траектории, лежашей на двумерном торе (т.е. на двумерном многообразии), на котором не может быть хаоса ввиду теоремы Пуанкаре-Бендиксона. (Эта теорема утверждает, что в ограниченной области двумерного пространства не может быть хаотического потока.) Однако Ньюхаус, Рюэль и Такенс показали (в 1978 году), что сушествование странного аттрактора не только возможно, но и характерно (т.е. практически неизбежно после двух бифуркаций Хопфа).

Более точно, Ньюхаус, Рюэль и Такенс показали (в 1978 году), что в системе, описывающей пространственный поток с тремя несоизмеримьми частотами, сколь угодно малое изменение приводит к тому, что этот квазипериодический поток становится хаотическим. Наивная логика подсказьвает (см., например, книгу Шустера [189]; см. также [190]), что потоки с тремя частотами маловероятны, так как они могут быть разрушены малыми возмушениями!

Однако численно было показано, что добавление гладких нелинейных возмущений, как правило, не разрушает трехчастотную квазипериодичность.

Разгадка в том, что в доказательстве Ньюхауса, Рюэля и Такенса малые возмущения, приводящие к хаотическим аттракторам, имеют малые первые и вторые производные, однако производные более высокого порядка, вообще говоря, не малы, как того требуют физические приложения!

Переход к турбулентности: сценарии, пути к хаосу. За первой бифуркацией могут последовать другие, и возникает естественный вопрос, что будет после некоторой серии бифуркаций. Принципиально существует бесконечно много возможностей, однако в некотором смысле, который уточняется ниже, не все из них равновероятны. Наиболее вероятные возможности называются сценариями, три из которых - самые известные и уже имеющие теоретические и экспериментальные подтверждения - изучаются ниже. В общем случае в сценарии описьваются аттракторы. Однако динамическая система может иметь много аттракторов. Поэтому различные сценарии могут одновременно развиваться в разных частях фазового пространства. Заметим, что сценарии не содержат информацию об областях их применимости!

“Неадекватный” сценарий Ландау-Хопфа. После первой бифуркации движение в обшем случае становится периодическим, после второй-квазипериодическим с двумя частотами и т. д. Было показано, что если первая бифуркация приводит к замкнутой орбите, то в результате второй бифуркации в фазовом пространстве может появиться притягивающий инвариантный двумерный тор. Если при этом движение таково, что его орбита плотна на этом торе, то полученные функции времени такие, как координаты в фазовом пространстве, квазипериодичны с двумя частотами. Более точно, мы можем определить две внутренние угловые координаты на двумерном торе и записать рассматриваемое движение в виде $\theta=\omega^{*} t+$ const, $\varphi=\omega^{* *} t+$ const. Соответствующая орбита плотна на торе тогда и только тогда, когда частоты $\omega^{*}$ и $\omega^{* *}$ несоизмеримы. После следуюшей бифуркации может возникнуть движение на трехмерном торе и т. д. Основная идея, стояшая за сценарием Ландау-Хопфа, состоит в том, что если имеется много независимых частот, то движение настолько нерегулярное, что в некоторых приложениях его можно рассматривать как хаотическое. Существуют различ- 
ные ситуации, когда этот сценарий непригоден. 1) Одна из бифуркаций может быть субкритической. В этом случае как только управляюший параметр $\lambda$ превьшшает соответствуюшее критическое значение, система перестает обладать движением, близким к устойчивому, и имеет место так назьваемый взрывной переход к движению, связывающему более или менее отдаленные части фазового пространства. 2) Хотя в общем случае после второй бифуркации появляется инвариантный двумерньй тор, орбита не обязана быть плотной на нем и может вернуться в начальную точку после конечного числа оборотов вокруг тора. В этом случае орбита замкнута, а движение является периодическим. В настояшее время ввиду теоремы Пейксото сушествует гипотеза, согласно которой замкнутые орбиты на двумерном торе менее вероятны, чем плотные. Это может привести к сценарию Фейгенбаума. 3) В ситуации, обсуждавшейся Рюэлем и Такенсом, после нескольких бифуркаций в фазовом пространстве возникает инвариантное множество, не являюшееся тором и назьваемое странньм аттрактором. Как объясняется ниже, движение при этом является не периодическим, а апериодическим.

Сценарий Рюэля-Такенса-Ньюхауса. В сценарии ранней стадии турбулентности, предложенном Рюэлем и Такенсом [176], предполагается, что первая бифуркация, как и в сценарии Ландау-Хопфа, является суперкритической и приводит к инвариантным торам $T^{k}, k=1,2,3,4$, каждый из которых представляет собой притягивающее множество с момента своего возникновения до следуюшей бифуркации. Сушествование торов обсуждается ниже при рассмотрении сценария Фейгенбаума. Рюэль и Такенс доказали, что на торе $T^{4}$ вполне вероятно движение по принадлежащему $T^{4}$ странному аттрактору определенного типа. Локально странный (хаотический) аттрактор является декартовым произведением двумерного канторова множества и двумерной поверхности. Векторные поля, порождающие странные аттракторы, нельзя считать редкими исключениями. Однако выбор странного аттрактора связан с некоторым произволом: легко представить себе многочисленные вариации, обладающие указанным вьше свойством. По-видимому, пока нет конкретных примеров векторных полей на заданном многообразии, которые приводят к странному аттрактору в точности по сценарию Рюэля-Такенса. Важной идеей в их статье является то, что движения по странным аттракторам в некотором смысле вполне вероятны или по крайней мере не исключены и, может быть, даже характерны при определенных обстоятельствах. В их теореме не утверждается, что существование странного аттрактора - типичное свойство векторного поля на $T^{4}$. Она просто утверждает, что если установлено существование инвариантного тора $T^{4}$, то движение по странному аттрактору более вероятно, чем квазипериодическое движение на $T^{4}$. Странным является аттрактор, на котором движения неустойчивы по Ляпунову и, следовательно, характеризуются непрерьвным энергетическим спектром. В действительности странность хаотического аттрактора устойчива при малых возмушениях динамической системы; другими словами, странность - не исключительное свойство.

Согласно Экману [191], если при изменении управляющего параметра система испьтывает три бифуркации Хопфа, начинающиеся со стационарного решения, то вполне вероятно, что после третей бифуркачии система будет обладать странныцм аттрактором с чувствительной зависимостью от начальных условий. В энергетическом спектре такой системы обнаруживается сначала одна, затем две, а возможно и три независимые частоты. Когда третья частота стоит на пороге 
появления, одновременно возникает иирокополосный шум, если существует странный аттрактор. Такое явление интерпретируется как хаотическая, турбулентная эволюция системы. Сценарий Рюэля-Такенса-Ньюхауса (см. [192]) не разрушается при добавлении к эволюционным уравнениям малого внешнего шума. Природа хаотических систем может быть совершенно нечувствительной к такому шуму. По-видимому, наиболее чувствительньми к шуму являются детерминированные системы вблизи точек перехода (бифуркации). На самом деле хаос в сценарии настолько сильный, что случайное установление порядка при добавлении малого шума невозможно, подобно тому, как шум локально не имеет сильного влияния на притягиваюшую неподвижную точку, а глобально может быть лишь малая вероятность стохастического перехода из одной области притяжения в другую. (Для аттрактора $A$ область притяжения определяется как множество точек $\boldsymbol{X}$, образ $F^{t} \boldsymbol{X}$ которого под действием потока $F^{t}$ стремится к $A$ при $t \rightarrow \infty$.) Хотя поток $F^{t}$ (диссипативной системы) сжимает объемы, он не обязан сжимать длины. В частности, если даже любое конечное множество $V$ фазового пространства $\mathbb{R}^{n}$ под действием потока стремится к единственному аттрактору $A$, могут сушествовать точки, принадлежашие аттрактору и сколь угодно близкие в начальньй момент, которые по истечении достаточно большого промежутка времени окажутся макроскопически отделенными (чувствительная зависимость от начальных условий). Такой аттрактор, сушествование которого не исключается даже в случае потоков, сжимающих площадь, называется странным. Решения диссипативных динамических систем, имеющих положительный характеристический показатель Ляпунова, могут экспоненциально расходиться. При этом движение является хаотическим, и появляется странный аттрактор. Характеристический показатель Ляпунова $\sigma$ потока $F^{t}$ определяется как предел

$$
\sigma=\lim \left\{\frac{1}{t} \ln \frac{D(t)}{D(0)}\right\} \text { при } t \rightarrow \infty \quad \text { и } D(0) \rightarrow 0
$$

где $D(t)$ - расстояние между решениями с близкими начальными условиями. Это дает меру средней экспоненциальной скорости расходимости двух решений с близкими начальными условиями или, по-другому, меру хаотичности турбулентности. Важно заметить, что величина $\sigma$ положительна, а это указьвает на хаотичность движения. Можно ожидать, что когда управляющий параметр (например, число Рейнольдса) возрастает, движение становится более хаотичным, что проявляется в увеличении показателя Ляпунова $\sigma$.

Наряду с показателем Ляпунова для описания турбулентности вводится еще одна величина, а именно, размерность странного аттрактора, на котором она (турбулентность) возникает. Эта размерность должна быть мерой числа активных степеней свободь или активных мод турбулентности. Вопрос о том, какие моды являются активными, может оказаться очень сложным. Первоначально обсуждения гидродинамического хаоса были в целом основаны на описании геометрических образов странных аттракторов, что возможно лишь при малых размерностях (т.е. при возникновении хаоса). Некоторые другие методы, применимые к умеренно возмушенным системам, дает эргодическая теория дифференииально-динамических систем (из условия эргодичности следует, что траектория равномерно покрывает энергетически допустимую область классического фазового пространства, так что среднее по времени 
можно заменить средним по соответствуюшему фазовому пространству). С эргодичной мерой $\rho$ ассоциируются разные параметры: а) характеристические показатели $\lambda_{1} \geqslant \lambda_{2} \geqslant \lambda_{3} \geqslant \cdots$ (назьваемые также показателями Ляпунова) из мультипликативной эргодической теоремы (Оселедца); $\lambda_{1}$ представляет собой скорость экспоненциальной расходимости орбит динамической системы с близкими начальными условиями; b) энтропия $H(\rho)$ - средняя скорость образования информации в системе или инвариант Колмогорова-Синая; с) информационная размерность $\operatorname{dim}_{H} \rho$ - наименьшая хаусдорфова размерность множеств $E$, удовлетворяюших условию $\rho(E)=1$.

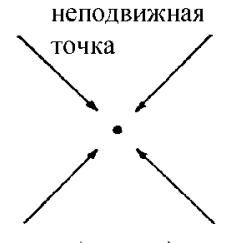

$(-,-,-)$

тор

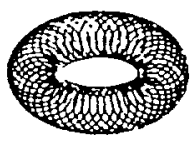

$(0,0,-)$

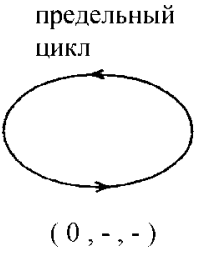

странный аттрактор

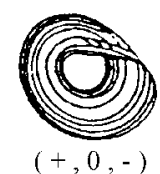

Рис. 4. Связь между размерностями простых аттракторов, вложенных в трехмерное фазовое пространство, и знаками соответствуюших им трех показателей Ляпунова (нуль означает, что показатель Ляпунова равен нулю)

Если система является эргодической, то энтропия задается формулой

$$
(H \rho)=\sum_{i=1}^{N} \lambda_{i},
$$

где сумма берется по всем положительныцм $\lambda_{i}$.

Размерность является, пожалуй, наиболее фундаментальньм свойством аттрактора. Существуют два общих типа определений размерности, один из которых зависит от метрических свойств, а другой - от частоты, с которой типичная траектория оказьвается в различных частях аттрактора.

Мы определим размерность $d$ хаотического аттрактора по формуле

$$
d=N-\sum_{i=1}^{N} \frac{\lambda_{i}}{\lambda_{N+1}}
$$

где

$$
\sum_{i=1}^{N+1} \lambda_{i}<0, \quad \sum_{i=1}^{N} \lambda_{i} \equiv H(\rho) \geqslant 0 .
$$


Заметим, что

$$
0<\sum_{i=1}^{N} \frac{\lambda_{i}}{\lambda_{N+1}}<1 .
$$

Для странного (хаотического) аттрактора Лоренца имеем $d=2.06$. Размерность аттрактора дает возможность оценивать число степеней свободы, которые участвуют в динамическом движении. При этом если размерность $d$ аттрактора мала и не является цельмм числом, то это свидетельствует о том, что динамика допускает детерминированное математическое описание, причем редуцированная система имеет невысокую размерность и характеризуется странньм аттрактором. Наконец, отметим, что в случае конечномерной динамической системы

$$
\frac{d X_{i}}{d t}=F_{i}, \quad i=1,2,3, \ldots, N,
$$

диссипативность означает, что

$$
\operatorname{div} F_{i}=\sum_{i=1}^{N} \frac{\partial}{\partial X_{i}} \frac{d X_{i}}{d t}<0 .
$$

Для системы Лоренца (8.6) ввиду неравенств $\sigma>0$ и $b>0$ имеем

$$
\operatorname{div} F_{i}=-(\sigma+1+b)<0,
$$

откуда следует, что обғемы сжимаются әкспоненциально по времени:

$$
V(t)=V(0) \exp [-(\sigma+1+b)]
$$

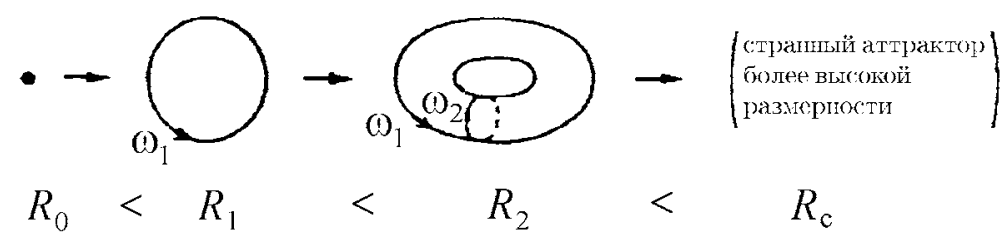

Рис. 5. Путь к хаосу Рюэля-Такенса ( $R$ - управляюший параметр, а $R_{c}-$ критическое значение $R$ )

Сценарий Фейгенбаума. В то время как аттрактор Лоренца возникает в связи с субкритической бифуркацией Хопфа, в сценариях Ландау-Хопфа и Рюэля-ТакенсаНьюхауса предполагается, что имеется последовательность суперкритических бифуркаций, приводящих к инвариантным торам все более высокой размерности, причем в первом сценарии размерности сколь угодно высоки, а во втором - не меньше 4. Однако существование такой последовательности маловероятно в силу теоремы Пейксото.

Фейгенбаум [193] предложил сценарий, основанньй на последовательности субгармонических бифуркаций с удвоением периодов (в этом случае инвариантный тор, 
вообще говоря, не существует, и в результате бифуркации могут возникнуть одна или несколько периодических орбит). Оказывается, что такие удвоения встречаются во многих примерах итерированных отображений и простых динамических систем. При этом, когда число удвоений $n$ возрастает, поведение системы управляется определенными асимптотическими законами, содержашими универсальные постоянные и функции, не зависящие от рассматриваемой системы. Более того, асимптотические законы довольно точно выполняются уже при сравнительно небольших значениях $n$. В частности, значения $\mu_{n}$ безразмерного параметра $\mu$, при которых происходят бифуркации (удвоения), сходятся к некоторому $\mu_{\infty}$ как геометрическая прогрессия, причем

$$
\frac{\mu_{n+1}-\mu_{n}}{\mu_{n}-\mu_{n-1}} \approx 0.21416938 \ldots \text { при больших } n \text {. }
$$

Когда $n \rightarrow \infty$, по крайней мере в изученных случаях, энергетический спектр движения приближается к непрерывному спектру с определенными универсальными свойствами. По-видимому, при $\mu=\mu_{\infty}$ движение на (странном) аттракторе апериодично.

Примером такого поведения является система Лоренца (8.6) при значениях безразмерного параметра $r$, сушественно превосходяших рассмотренные Лоренщем значения. А именно, странный аттрактор Лоренца, который возникает при $r=24.74$, сохраняется вплоть до некоторого значения $r=r^{*}(\approx 250)$. Когда $r$ значительно больше $r^{*}$, сушествует периодическая орбита, и когда $r^{*}$ убывает в направлении $r^{*}$, появляется последовательность удвоений при определенных значениях $r_{n}$ параметра $r$, которые сходятся к $r^{*}$ сверху, причем

$$
\frac{r_{n+1}-r_{n}}{r_{n}-r_{n-1}} \approx 0.21416938 \ldots .
$$

Существует универсальное число (Фейгенбаума)

$$
\frac{1}{0.21416938 \ldots}=4.669201545 \ldots=\delta
$$

такое, что

$$
\left|\mu_{j}-\mu_{\infty}\right| \approx \operatorname{const} \delta^{-j} \text { при } j \rightarrow \infty .
$$

После каскада удвоений периодов можно ожидать, что за точкой накопления $\mu_{\infty}$ возникает обратный каскад шумовых периодов. Если субгармонические бифуркации экспериментально наблюдаются при значениях $\mu_{1}$ и $\mu_{2}$, то согласно сценарию Фейгенбаума сушествует большая вероятность того, что бифуркация произойдет около точки

$$
\mu_{3}=\mu_{2}-\frac{\mu_{1}-\mu_{2}}{\delta} .
$$

$\mathrm{K}$ тому же, если уже произошли три бифуркации, то четвертая бифуркация становится более вероятной, чем третья бифуркация после второй, и т. д. В точке накопления наблюдается апериодичное поведение, но широкополосный спектр отсутствуem.

Сценарий Фейгенбаума исключительно хорошо согласуется с численными и физическими экспериментами. Удвоения периодов наблюдаются во многих динамических 


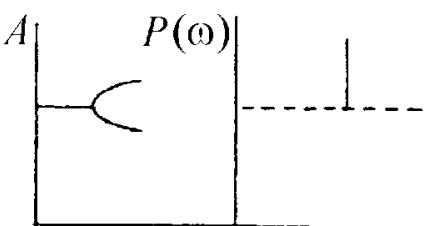

$\mu$

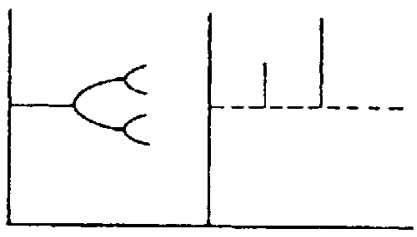

$\omega$
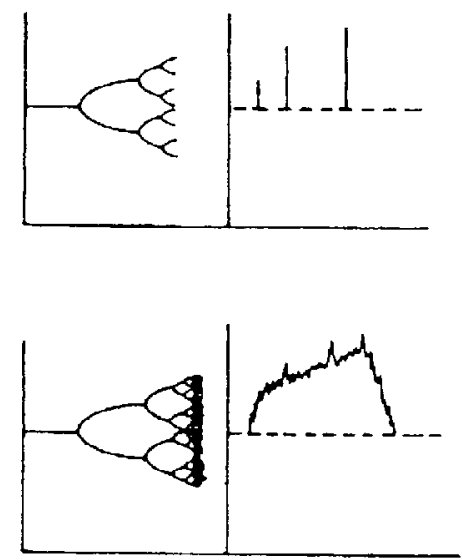

Рис. 6. Энергетические спектры и бифуркационные диаграммы для сценария Фейгенбаума

системах малой размерности, и это явление изучалось с помошью анализа группы ренормализации. Отметим, что используемая в этих случаях функциональная группа ренормализации строится по аналогии с методом группы ренормализации для критических явлений (см., например, статьи Аргула и Арнеодо (с. 241-288) и Кулле и Трессера (с. 217-240) в сборнике [194]).

В статье Эррафия и Зейтуняна [179] можно найти пример перехода к хаосу по сценарию Фейгенбаума для так назьваемой задачи Бенара о глубокой конвекиии. В этом случае имеется последовательность периодических режимов с удвоением периодов (при шести значениях управляющего параметра: $\mu=200,217.5,219.5,220,240$ и 250 ). На первом из приводимых ниже рисунков представлены фазовые диаграммы для $\mu=$ 220,240 и 250. Хаос возникает при $\mu=270$, и на втором рисунке приведен соответствуюший хаотический аттрактор Фейгенбаума для $\mu=290$. Строго говоря, этот хаотический аттрактор Фейгенбаума не является странным (точное определение этого объекта можно найти в [189; гл. 5]), однако непрерывность энергетического спектра очень характерна для хаоса. Таким образом, в рассматриваемом случае при переходе к хаосу происходят последовательные (субгармонические) бифуркации удвоения периода от (простого) периодического потока (соответствующего $\mu=200$ ).

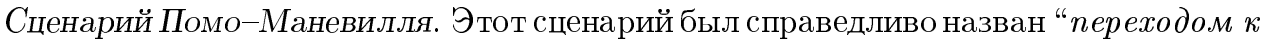
турбулентности через перемежсаемость". Его математическое состояние по сравнению с двумя другими приведенными вьше сценариями - менее удовлетворительное. Это связано с тем, что область изменения параметров, при которых описьвается сценарий, содержит бесконечное число (очень длинных) устойчивых периодов, и при этом не уточняется, когда достигается “турбулентньй режим" и какова точная природа этой турбулентности!

В отличие от сценариев Рюэля-Такенса-Ньюхауса и Фейгенбаума, ассоциированных соответственно с бифуркациями Хопфа и вильными бифуркациями, сценарий Помо-Маневилля связан с "седло-узловой бифуркацией", т.е. со столкновением устойчивой и неустойчивой неподвижньх точек, которые затем исчезают (пре- 

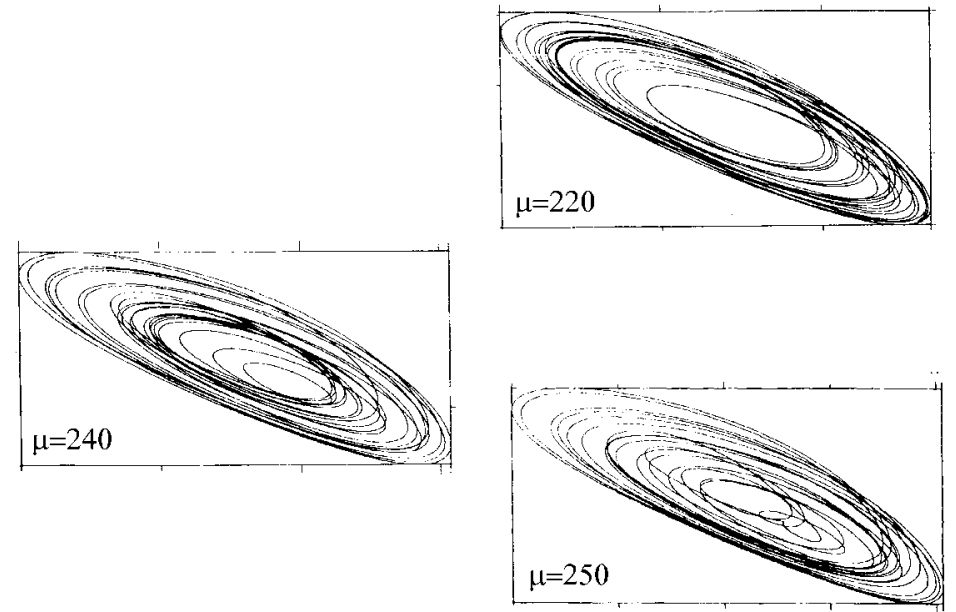

Рис. 7. Фазовые диаграммы при $\mu=220,240$ и 250

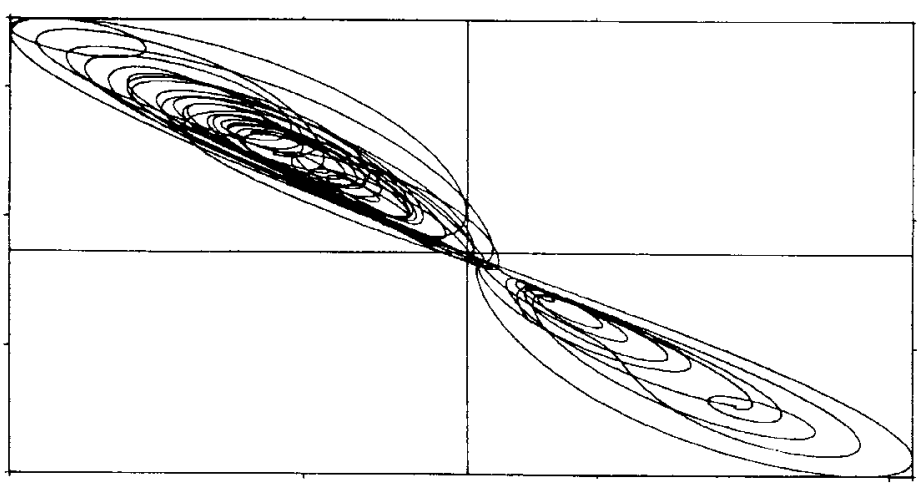

Рис. 8. Аттрактор Фейгенбаума при $\mu=290$

врашаются в комплексные неподвижные точки). Под перемежаемостью мы подразумеваем появление сигнала, который случайным образом альтернирует между длинными регулярньми (ламинарными) фазами (так назьваемые паузы) и относительно короткими нерегулярными импульсами. Было установлено, что с увеличением внешнего параметра число хаотических импульсов возрастает, а это означает, что переход от регулярного к хаотическому движению через перемежаемость является непрерывным. Переход к хаосу через перемежаемость обладает на самом деле универсальными свойствами и является одним из редких примеров, когда (линеаризованные) уравнения группы ренормализации допускают точное решение. Этот путь к хаосу был изучен в пионерской работе Манневилля и Помо [195]. Они численно решили дифференциальные уравнения модели Лоренца (8.6) и обнаружили, что при $r<r_{c}$ компонента $Y(t)$ осуществляет устойчивое периодическое движение. Выше порога $r_{c}$ осцилляции прерьваются хаотическими импульсами, которые с увеличением $r$ учашаются до тех пор, пока движение не становится полностью хаотическим. В действительности устойчивые осцилляции при $r<r_{c}$ соответствуют устойчивой неподвижной точке 
отображения Пуанкаре. При $r>r_{c}$ эта неподвижная точка становится неустойчивой. Так как в общем случае существуют три возможные пути потери устойчивости, Помо и Манневилль рассмотрели три случая перемежаемости (типы I, II и III; см. [189; гл. 4]). Например, в модели Лоренца имеет место перемежаемость типа I, причем в этом случае переход к хаосу характеризуется обратной тангенииальной бифуркацией; после исчезновения пары устойчивой и неустойчивой неподвижных точек, итерации долгое время остаются в "канальной области” (между графиком отображения и биссектрисой). Это приводит к длинной ламинарной области для значений $r$, примыкаюших к $r_{c}$ сверху, и после того, как траектория покидает канал, движение становится хаотическим до повторного вхождения в окрестность “фиктивной неподвижной точкой” (которая притягивает траектории слева и отталкивает их справа) и начала новой регулярной фазы.
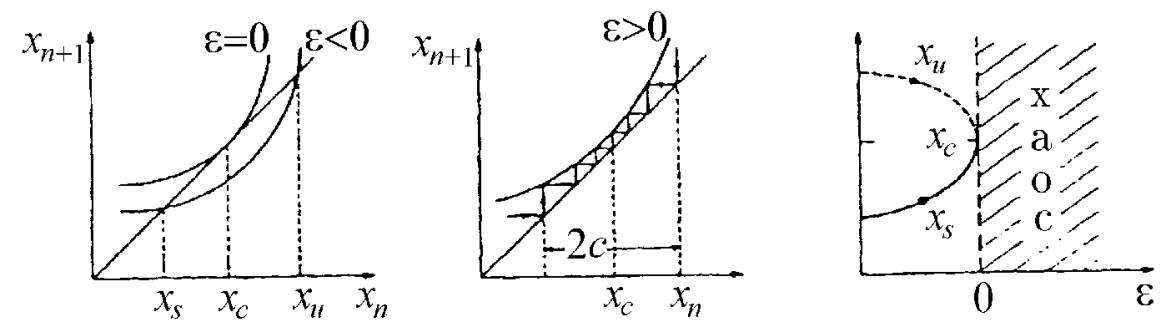

Рис. 9. Механизм перемежаемости типа I: отображения Пуанкаре при $\varepsilon=$ $r-r_{c} \leqslant 0$ и $\varepsilon>0$; движение траектории при $\varepsilon>0$; обратная тангенциальная бифуркация

Теория Помо-Манневилля объясняет только ламинарное течение, но не дает информации о механизме рождения хаоса! Следует отметить, что тангенциальные бифуркации (в отличие от вильных бифуркаций, в результате которых число неподвижных точек удваивается) являются единственным механизмом, благодаря которому на логистической карте появляется нечетное число неподвижных точек.

Перемежаемость типа III возникает одновременно с бифуркацией удвоения периода. Наблюдается рост субгармонической и убывание фундаментальной амплитуд. Когда субгармоническая амплитуда достигает высоких значений, сигнал теряет регулярность и появляются турбулентные импульсы. В случае перемежаемости типа II периодические (но неустойчивые) траектории продолжают существовать после возникновения неустойчивости. Таким образом, в отличие от перемежаемости типа I максимальная продолжительность кажущихся периодическими осцилляций между двумя импульсами не ограничена сверху, и даже в режиме развитой турбулентности последовательности регулярных осцилляций продолжают время от времени появляться. Отметим наконец, что при дальнейшем варьировании параметра от критического значения $\mu_{c}$ к $\mu$ выявляется перемежаемость интервалов турбулентного поведения случайной продолжительности и ламинарных фаз средней продолжительности $\sim\left|\mu-\mu_{c}\right|^{-1 / 2}$.

На приводимых ниже рисунках представлены энергетические спектры и аттракторы (для двух значений управляющего параметра $\varepsilon: \varepsilon=125$ и 130) в случае задачи Бенара о глубокой конвекиии (согласно [179]). Уточним, что при $\varepsilon=120$ имеет мес- 
то чисто периодический режим. В этом случае неустойчивость возникает через перемежающийся режим, и при $\varepsilon=130$ аттрактор является хаотическим. Это крайне “захватьваюшее” численное наблюдение (М. Эррафия) перехода к хаосу через перемежаемость очень хорошо согласуется со сценарием Помо-Манневилля. При $\varepsilon=130$ начинается временная эволюция, которая случайным образом альтернирует между относительно короткими псевдорегулярными (псевдоламинарными) фазами (так называемые паузы) и “относительно большими” нерегулярными импульсами. Число хаотических импульсов возрастает с увеличением $\varepsilon$ (в рассматриваемом случае от 122 до 130), а это означает, что переход от регулярного движения к хаотическому через перемежаемость является непрерьвным.
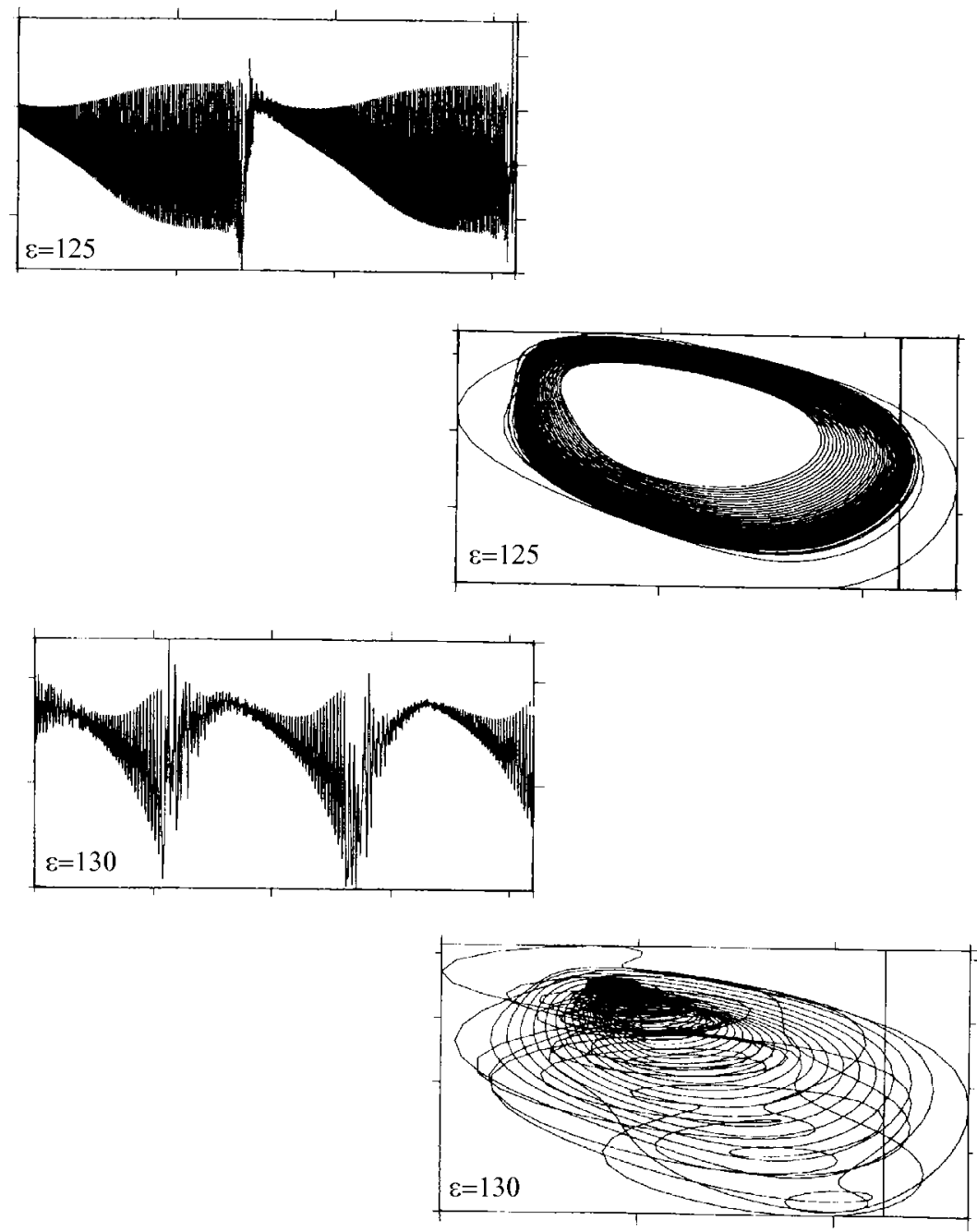

Рис. 10. Численные свидетельства перемежаемости. При $\varepsilon=130$ импульсы относительно велики 
Теория бифуркаиий представляет собой, по сушеству, теорию равновесных решений нелинейных уравнений. Под равновесными решениями мы подразумеваем, например, стационарные, периодические или квазипериодические по времени решения. Целью книги Йоосса и Джозефа [196] является исследование теории бифуркаций равновесных решений эволюционных задач, управляющихся нелинейными дифференциальными уравнениями. Интересно отметить, что общая теория проще, чем подробная теория в конкретных приложениях! Общепризнанно, что математическая теория бифуркаций требует привлечения функционального анализа и некоторых методов топологии и динамических систем. В работах [196] и [197] основным приложением функционального анализа в теории бифуркаций является обоснование редукции задач в пространствах большой или бесконечной размерности к одномерным или двумерньм задачам. Возникаюшие на этом пути задачи малой размерности связаны с проекторами на подпространства, порожденные собственными функциями, причем в некоторых специальных случаях, таких как вырожденные задачи о стационарных бифуркациях с разрушением симметрии, редуцированная задача может иметь размерность больше чем два.

Существует $m p и$ основных метода для получения задач в пространствах малой размерности. В некоторых работах используется "метод Ляпунова-Шмидта" для расшепления уравнений и соответствуюшего пространства решений на конечномерную и бесконечномерную компоненты (см., например, [187]). Бесконечномерную часть уравнения можно решить, а полученная конечномерная задача содержит всю информацию о бифуркации. Подробное математическое обсуждение этого метода можно найти в статье Вайнберга и Треногина [198]. В ряде работ для получения конечномерных задач используется метод “иентрального многообразия". Этот метод основан на том наблюдении, что в задачах, подобных изученным в [196], решения притягиваются к центральному многообразию конечной размерности. Полное описание этого метода и вывод амплитудных уравнений можно найти в [199] и [200]. Указанные два метода пригодны для доказательства теорем существования.

Последним (третьим) методом является “теорема о неявной функции”, которая используется для обоснования прямого, последовательного вычисления коэффициентов разложения решений в степенные ряды по (малой) амплитуде $\varepsilon$, причем для этого привлекается также альтернатива Фредгольма. Систематическое изложение этого подхода можно найти в [196].

Обсуждение вопросов, связанных с нелинейной динамикой и турбулентностью, можно найти в сборнике [201]. Многие статьи этого сборника посвящены разъяснению обобщенной конщепии турбулентности и исследованию ее приложений в задачах турбулентности в жидкости. Для этого комбинируются аналитические, численные и экспериментальные методы.

Интересно также отметить, что если бифуркации происходят в параметризованных динамических системах, когда изменение (управляющего) параметра приводит к расшеплению равновесного состояния на два таких состояния, то возникает катастрофа, при которой нарушается устойчивость равновесия, в результате чего система перескакивает в другое состояние. В работе Зеемана [202] описьваются некоторые странные аттракторы и странные бифуркации, а также иллюстрируется разница между бифуркацией и катастрофой в "неэлементарной" теории. Зееман обсуждает также некоторые связанные $c$ турбулентностью свойства, такие как 
устойчивость, чувствительная зависимость от начальных данных и широкополосные частотные спектры, аналогичные тем, которые рассмотрены в работе Свинни и Голлуба [203]. Короткий, но в то же время содержательньй и поучительный нематематический обзор менее спорных результатов дается в книге Арнольда [204], которая может служить полезньм введением в эту область. Целью книги [205] является обсуждение математических идей при помощи их визуального изложения. Эта книга основана на том, что математические понятия (такие как критические точки в трехмерном пространстве, предельные циклы, характеристические показатели, энергетические спектры, хаотические предельные множества и свойства хаоса) можно легко связывать друг с другом, согласовывая визуальные, словесные и символические представления, что дает возможность исследовать математические задачи с привлечением визуальных моделей.

С другой стороны, изучение асимптотического поведения решений диссипативных дифференциальных уравнений с частными производными при больших временах является главной задачей математической физики, которая тесно связана с пониманием турбулентности. В общем случае поведение динамических систем на бесконечности можно описывать с помощью глобальных аттракторов. С этим объектом тесно связано понятие приближенного инерииального многообразия, введенное в работе Фойаша, Менли и Темама [206]. Напомним, что приближсенное инерииальное многообразие является конечномерным гладким многообразием, в малую окрестность которого за конечное время попадает любое решение. В частности, глобальный аттрактор содержится в инерииальном многообразии. Существование приближенного инерциального многообразия установлено для многих диссипативных дифференциальных уравнений в частных производных (см., например, статью Темама [207]). Более точно, асимптотическое поведение решений диссипативных эволюционных уравнений при больших временах описьвается компактным аттрактором, притягивающим все ограниченнье множества. Современное состояние теории динамических систем (см. [208]) не дает много информации о геометрии аттракторов, которые могут быть фрактальными множествами [199], [209]. В силу общих теорем, которые применимы к различным динамическим системам, аттрактор имеет ${ }^{5}$ конечную хаусдорфову размерность и конечную емкость (фрактальную размерность); см., например, [211]. В работе [208] предложен метод аппроксимации глобальных аттракторов диссипативных дифференциальных уравнений (включая двумерные уравнения Навье), основанньй на аналитичности по времени (в фиксированной бесконечной полосе) всех решений, лежащих на аттракторе. В частности, строятся семейства полиномиальных отображений, нули которых при соответствующих условиях аппроксимируют аттрактор. Некоторые другие приближения аттракторов конечномерными алгебраическими или аналитическими множествами построены в статье [207], однако там эти множества являются графиками над конечномерньпи подпространствами! Стоит отметить, что система Лоренца (8.6) с обычными параметрами $\sigma=10$, $b=8 / 3$ и $r=28$ не удовлетворяет условиям, наложенным в $\S \S 3,7$ работы [208].

Если при рассмотрении двумерных уравнений Навье-Стокса ограничиться галеркинскими приближениями (общее изложение можно найти, например, в [212]),

\footnotetext{
5Это утверждение справедливо в случае автономных динамических систем. Хаусдорфова размерность аттрактора неавтономного уравнения может быть бесконечной [210]. Прим. перев.
} 
которые согласованы с подпространствами $V_{m}$, натянутыми на первые $m$ собственных функций $w_{j}$ ассоциированной (линейной) задачи Стокса, то для достаточно больших $m$ асимптотика при $t \rightarrow \infty$ галеркинской аппроксимации $u_{m}$ полностью определяется поведением при $t \rightarrow \infty$ некоторого фиксированного числа $m^{*}$ ее мод (см. [201; с. 139-155]). Интересно отметить, что, как показано в статье Трева [201; с. 334-342], важные свойства галеркинских приближений легко устанавливаются при условии, что они удовлетворяют уравнениям әнергетического равновесия. Это еще одно оправдание рассмотрения таких аппроксимаций в задачах гидродинамики, и во многих случаях они действительно могут быть построены. Для классической задачи Бенара о конвекции Трев показал, что сушествует единственньй способ построения галеркинских приближений любого порядка, для которых уравнения энергетического равновесия выполняются точно. Более того, он дал простое доказательство ограниченности таких аппроксимаций, показал, как получить оценки для физически интересных величин, таких как число Нуссельта, и установил глобальную устойчивость чисто проводящих решений для субкритических чисел Рейнольдса. Фойаш и Со [213] показали, что для эволющионных уравнений Навье отношение энтропии и энергии имеет предел при $t \rightarrow \infty$, которьй является собственным значением ассоциированного оператора Стокса. Этот факт имеет прямые следствия, касающиеся глобальной структуры уравнений Навье. В самом деле, он позволяет построить в пространстве начальных данных $R$ фолаг нелинейных спектральных многообразий оператора Навье. Это конечномерные аналитические многообразия, которые инвариантны относительно уравнений Навье и полностью описьвают убьвание энергии решений. Статья Гидалья [214] была мотивирована изучением аттракторов и поведения решений при больших временах для бесконечномерных динамических систем, возникаюших в гидромеханике (уравнения Навье, магнитогидродинамические и термогидравлические уравнения), в теории горения (уравнения Курамото-Сивашинского) и в оптике (нелинейное уравнение Шрёдингера).

Наиболее современньй анализ уравнений Навье можно найти в [15], [20], [211], [215] и [216]. Отметим, что книга Доеринга и Гиббона [216] содержит изложение математической и физической основ уравнений Навье-Стокса, причем особое внимание уделяется нерешенньм задачам, касаюшимся регулярности решений в трехмерном случае. Авторам удалось дать всестороннее представление о сильных и слабых сторонах методов, используемых при исследовании этих уравнений.

В недавней книге Трошкина [217] излагаются специальные методы, используемые в гидродинамике. "Нетрадиционность", упомянутая в заглавии книги, возможно, состоит в том, что наряду с традишионными методами анализа используются идеи из теории динамических систем, а также некоторые геометрические и топологические методы. Однако с течением времени любые методы становятся традиционными!

Новейшие результаты, касаюшиеся ламинарно-турбулентных переходов, можно найти в материалах [218] четвертого симпозиума IUTAM, состоявшегося в Сендае с 5-го по 9-е сентября 1994 года. “Асимптотические подходы моделирования переходов" обсуждаются в обзоре Каули и Ксуесонг Ву [219]. Вопросы структуры и статистики турбулентности представлены в материалах симпозиума, состоявшегося 17-18 января 1994 года в Исследовательском институте математических наук университета Киото (см.: Math. Reviews, 96h:76002).

В лекциях [220] читатель может найти разные интересные и красивые цветные ил- 
люстрации возникновения фракталов, как хаотических множеств динамических систем. Введение в хаотическую динамику можно найти также в книге Бейкера и Голлуба [221], которая является не исследовательской монографией, а скорее очень содержательным начальньм курсом, касающимся фундаментальных явлений. Основное внимание при этом уделяется не полноте изложения, а методам теории. В книге Арефа и Эль Наши [222] приводится всесторонний обзор современного состояния хаотической адвекиии, покрывающий многочисленные аспекты, в том числе математические методы, используемые при описании хаотической адвекции, детальное изучение основной динамики, апериодические системы, растяжение и синхронизация, хаотические адвекция и диффузия. В книге Бека и Шлёгла [223] обсуждаются разные термодинамические концепции, используемые при анализе нелинейных динамических систем. Недавняя книга Марека и Шрейбера [224] представляет собой продвинутый учебник о теоретических и экспериментальных аспектах хаотического поведения. Книга [225], выпушенная под редакцией Годрехе и Манневилля, содержит пять курсов лекций всемирно признанных ученых на темы нелинейных неустойчивостей и перехода к турбулентности в гидродинамике. В книгеван Гроезена и Егера [226] дается прекрасный обзор роли бесконечномерных гамильтоновых и пуассоновских динамических структур в ряде задач, возникаюших в уравнениях с частными производными. Примечательной особенностью является признание того, что гамильтоновый случай - лишь идеализированньй предел в гидромеханике.

Целью книги Николиса [227] является развитие единого подхода в нелинейной науке, показывающего ее многогранность, а также разнообразие и богатство концепший и методов, разработанных за многие годы.

Отметим, наконец, недавнюю книгу Кеворкяна и Коула [228], которая является переработанной и обновленной версией весьма известного труда авторов "Meтоды возмущений в прикладной математике". Большая часть нового издания написана заново и содержит много свежего материала, в том числе по одной главе о почти единичных усредняюших преобразованиях и о многомаситабных разложсениях для уравнений с частными производными.

\section{СПИСОК ЛИТЕРАТУРЫ}

[1] Hadamard J. La Théorie des Équations aux Dérivées Partielles. Pekin: Editions Scientifiques, 1964

[2] Meyer R. E. Introduction to Mathematical Fluid Dynamics. New York: Wiley, 1971.

[3] Серрин Дж. Математические основы классической механики жидкостей. М.: ИЛ, 1963.

[4] Прагер В. Введение в механику сплошных сред. М.: ИЛ, 1968.

[5] Zeytounian R.Kh. The Bénard problem for deep convection: rigorous derivation of approximate equations // Internat. J. Engrg. Sci. 1989. V. 27. №11. P. 1361-1366.

[6] Gustafsson B., Sundström A. Incompletely parabolic problem in fluid dynamics // SIAM J. Appl. Math. 1978. V. 35. P. 343-357.

[7] Oliger J., Sundström A. Theoretical and practical aspects of some initial boundary value problems in fluid dynamics // SIAM J. Appl. Math. 1978. V. 35. P. 419-446.

[8] Zeytounian R. Kh. The Bénard-Marangoni thermocapillary instability problem: on the role of the buoyancy // Internat. J. Engrg. Sci. 1997. V. 35. № 5. P. 455-466.

[9] Белов Ю. Я., Яненко Н. Н. Влияние вязкости на гладкость решений неполно-параболических систем // Матем. заметки. 1971. Т. 10. С. 93-99.

Автор благодарен переводчику, обратившему его внимание на работы [54], [176], [190], [210]. 
[10] Strikwerda J. C. Initial boundary value problems for incompletely parabolic systems // Comm. Pure Appl. Math. 1977. V. 30. P. 797-822.

[11] Ладыженская О. А. Математические вопросы динамики вязкой несжимаемой жидкости. М.: Наука, 1970.

[12] Shinbrot M. Lectures on Fluid Mechanics. New York: Gordon and Breach, 1973.

[13] Темам Р. Уравнения Навье-Стокса. Теория и численный анализ. М.: Мир, 1981.

[14] Антонцев С.Н., Кажихов А. В., Монахов В. Н. Краевые задачи механики неоднородных жидкостей. Новосибирск: Наука, 1983.

[15] Constantin P., Foias C. Navier-Stokes Equations. Chicago: Univ. of Chicago Press, 1988.

[16] von Wahl W. The Equations of Navier-Stokes and Abstract Parabolic Equations. Brunschweig: Vieweg, 1985.

[17] Majda A. Mathematical Foundations of Incompressible Fluid Flow. Princeton University, Department of Mathematics, Lecture Notes, 1985.

[18] Valli A. Mathematical results for compressible flows // In "Mathematical Topics in Fluid Mechanics" (Lisbon, 1991). Pitman Res. Notes Math. Ser., 274. Harlow: Longman Sci. Tech., 1992. P. 193-229.

[19] Рихтмайер Р. Принципы современной математической физики. Т. I, II. М.: Мир, 1982, 1984.

[20] Lions P.-L. Mathematical Topics in Fluid Mechanics, Vol. I. Incompressible Models, Vol. II. Compressible models. Oxford: Clarendon Press, 1996, 1998.

[21] Solonnikov V.A., Kazhikhov A. V. Existence theorems for the equations of motion of a compressible viscous fluid // Annual Rev. Fluid Mech. 1981. V. 13. P. 79-95.

[22] Beirão da Veiga H. On the barotropic motion of compressible perfect fluids // Ann. Scuola Norm. Sup. Pisa Cl. Sci. Ser. IV. 1981. V. 8. P. 317-351.

[23] Beirão da Veiga H. Perturbation theorems for linear hyperbolic mixed problems and applications to the compressible Euler equations // Comm. Pure Appl. Math. 1993. V. 46. №2. P. 221-259.

[24] Graffi D. Il teorema di unicità nella dinamica dei fluidi compressibili // J. Rational Mech. Anal. Math. 1953. V. 2. P. 99-106.

[25] Serrin J. On the uniqueness of compressible fluid motion // Arch. Rational Mech. Anal. 1959. V. 3. P. 271-288.

[26] Kato T. Quasi-linear equations of evolution, with applications to partial differential equations // Spectral theory and differential equations / ed. W.N. Everitt. Berlin: Springer-Verlag, 1975. P. 25-70.

[27] Sideris T.C. Formation of singularities in three-dimensional compressible fluids // Comm. Math. Phys. 1985. V. 101. P. 475-485.

[28] Secchi P. Existence theorems for compressible viscous fluids having zero shear viscosity // Rend. Sem. Mat. Univ. Padova. 1983. V. 70. P. 73-102.

[29] Valli A. Periodic and stationary solutions for compressible Navier-Stokes equations via a stability method // Ann. Scuola Norm. Sup. Pisa Cl. Sci. Ser. IV. 1983. V. 10. P. 607-647.

[30] Matsumura A. An energy method for the equation of motion of compressible viscous and heat-conductive fluids. MRC Technical Summary Report. № 2194. Madison: University of Wisconsin, 1981.

[31] Matsumura A., Nishida T. The initial boundary value problem for the equations of motion of compressible viscous and heat-conducting fluid. MRC Technical Summary Report. № 2237. Madison: University of Wisconsin, 1981.

[32] Matsumura A., Nishida T. Initial boundary value problems for the equations of motion of general fluids // Computing methods in applied sciences and engineering, $\mathrm{V}$ / ed. R. Glowinski, J.-L. Lions. Amsterdam: North-Holland, 1982. P. 389-406.

[33] Valli A., Zajaczkowski W. M. Navier-Stokes equations for compressible fluids: global existence and qualitative properties of the solutions in general case // Comm. Math. Phys. 1986. V. 103. P. 259-296. 
[34] Zajaczkowski W. M. On nonstationary motion of a compressible barotropic viscous capillary fluid bounded by a free surface // SIAM J. Math. Anal. 1994. V. 25. № 1. P. 1-84.

[35] Valli A. Uniqueness theorems for compressible viscous fluids, especially when the Stokes relation holds // Boll. Un. Mat. Ital. Ser. V. Anal. Funz. Appl. 1981. V. 18-C. P. 317-325.

[36] Itaya N. On the initial value problem of the motion of compressible viscous fluid, especially on the problem of uniqueness // J. Math. Kyoto Univ. 1976. V. 16. P. 413-427.

[37] Heywood J. G. The Navier-Stokes equations: on the existence, regularity and decay of solutions // Indiana Univ. Math. J. 1980. V. 29. № 5. P. 639-680.

[38] Simon J. Nonhomogeneous viscous incompressible fluids: existence of velocity, density and pressure // SIAM J. Appl. Math. 1990. V. 21. № 5. P. 1093-1117.

[39] Amick C.J. On steady Navier-Stokes flow past a body in the plane // Sympos. Pure Math. 1986. V. 45. № 1. P. 37-50.

[40] Leray J. Etude de diverses équations intégrales non linéaires et de quelques problèmes que pose l'hydrodynamique // J. Math. Pures Appl. (9). 1933. V. 12. P. 1-82.

[41] Nonlinear Functional Analysis and its Applications. Proc. Summer. Res. Inst., Berkley/Calif. 1983. Proc. Symp. Pure Math. V. 45. Pt. 1, 2, 1986.

[42] Valli A. Navier-Stokes equations for compressible fluids: global estimates and periodic solutions // Nonlinear Functional Analysis and its Applications. Proc. Summer Res. Inst., Berkeley/Calif. 1983. Proc. Symp. Pure Math. V. 45. Pt. 2, 1986. P. 467-476.

[43] Temam R. (ed. ) Navier-Stokes Equations and Turbulence. Berlin: Springer-Verlag, 1975. (Lecture Notes in Math. V. 565.)

[44] Temam R. On the Euler equations of incompressible perfect fluids // J. Funct. Anal. 1975. V. 20. P. 32-43.

[45] Baouendi M.S., Goulaouic C. Sharp estimates for analytic pseudodifferential operators and application to Cauchy problems // J. Differential Equations. 1983. V. 48. P. 241-268.

[46] Metivier G. Un théorème de Cauchy-Kowalevski pseudo-différentiel local // Commun. Partial Differential Equations. 1985. V. 10. P. 341-363.

[47] Bardos C. Analyticité de la solution de l'équation d'Euler dans un ouvert de $\mathbb{R}^{n} / / \mathrm{C}$. R. Acad. Sci. Paris. Sér. I Math. 1976. V. 283. P. 255-258.

[48] Bardos C., Benachour S., Zerner M. Analyticité des solutions périodiques de l'équation d'Euler en dimension deux // C. R. Acad. Sci. Paris. Sér. I Math. 1976. V. 282. P. 995-998.

[49] Benachour S. Analyticité des solutions périodiques de l'équation d'Euler en dimension trois // C. R. Acad. Sci. Paris. Sér. I Math. 1976. V. 283. P. 107-110.

[50] Alinhac S., Metivier G. Propagation de l'analyticité locale pour les solutions de l'équation d'Euler // Arch. Rational Mech. Anal. 1986. V. 92. P. 287-296.

[51] Le Bail D. Analyticité locale pour les solutions de l'équation d'Euler // Arch. Rational Mech. Anal. 1986. V. 95. № 2. P. 117-136.

[52] Ward G. N. Linearized Theory of Steady High-Speed Flow. Cambridge: Cambridge Univ. Press, 1955

[53] Курант Р., Фридрихс К. Сверхзвуковое течение и ударные волны. М.: ИЛ, 1950.

[54] Ландау Л. Д., Лифшиц Е. М. Теоретическая физика IV. Гидродинамика. М.: Наука, 1988.

[55] Germain P. Shock waves, jump relations, and structure // Adv. Appl. Mech. 1972. V. 12. P. 131-194.

[56] O'Neil M. E., Chorlton F. Viscous and Compressible Fluid Dynamics. Chichester/New York: Ellis Horwood Ltd./Halsted Press, 1989.

[57] Хейз У. Д., Пробстин Р. Ф. Теория гиперзвуковых течений. М.: Мир, 1962.

[58] Гиро ЖК. П. Основные вопросы теории гиперзвуковых течений. М.: Мир, 1965.

[59] Lagerstrom P. A. Laminar flow theory // Theory of Laminar Flows. Section B / ed. F. K. Moore. Princeton, NJ: Princeton Univ. Press, 1964. P. 20-285.

[60] François C. Les méthodes de perturbation en mécanique. Paris, 1981. (E. N. S. T. A. V. 507.) 
[61] Germain P., Guiraud J.-P. Conditions de choc et structure des ondes de choc dans un ecoulement non stationnaire de fluide dissipatif // J. Math. Pures Appl. 1966. V. 45. P. 311-358.

[62] Lagerstrom P. A. Matched Asymptotic Expansions. Ideas and Techniques. New York: Springer-Verlag, 1988.

[63] Kaplun S., Lagerstrom P. A. Asymptotic expansions of Navier-Stokes solutions for small Reynolds numbers // J. Rational Mech. Anal. 1957. V. 6. P. 585-593.

[64] Ван Дайк М. Д. Методы возмущений в механике жидкостей. М.: Мир, 1967.

[65] Guiraud J.-P., Zeytounian R. Kh. (ed. ) Asymptotic Modelling of Fluid Flows // J. Méc. Théor. Appl. Suppl. 1986.

[66] Zeytounian R. Kh. Modélisation Asymptotique en Mécanique des Fluides Newtoniens. Berlin: Springer-Verlag, 1994.

[67] Cercignani C. Une histoire de la théorie cinétique des gas. La vie des sciences // Comptes Rendus. Sér. Générale. 1992. V. 9. №4. P. 275-285.

[68] Guiraud J.-P., Zeytounian R.Kh. A double-scale investigation of the asymptotic structure of rolled-up vortex sheets // J. Fluid. Mech. 1977. V. 79. №1. P. 93-112.

[69] Guiraud J.-P., Zeytounian R. Kh. Une théorie pour le noyau de nappes tourbillonnaires à enroulement serré // Rech. Aérospat. 1977. V. 4 (Juillet-Aout). P. 205-212.

[70] Guiraud J.-P. The dynamics of rolled vortex sheets tightly winded around slender vortex filaments in inviscid incompressible flow // Lecture Notes in Math. 1977. V. 594. P. 244-259.

[71] Guiraud J.-P., Zeytounian R. Kh. A note on the viscous diffusion of rolled vortex sheets // J. Fluid Mech. 1979. V. 90. № 1. P. 197-201.

[72] Stewartson K., Hall M.G. The inner viscous solution for the core of a leading-edge vortex // J. Fluid Mech. 1963. V. 15. № 2. P. 306-318.

[73] Guiraud J.-P., Zeytounian R. Kh. Rotational compressible inviscid flow with rolled vortex sheets. An analytical algorithm for the computation of the core // J. Fluid Mech. 1980. V. 101. № 2. P. 393-401.

[74] Guiraud J.-P. Going on with asymptotics // Asymptotic Modelling in Fluid Mechanics / ed. P.-A. Bois, E. Dériat, R. Gatignol, and A. Rigolot. Berlin: Springer-Verlag, 1995. P. 257-307.

[75] Villat H. As luck would have it - a few mathematical reflections // Annual Rev. Fluid. Mech. 1972. V. 4. P. 1-5.

[76] Stewartson K. D'Alembert's paradox // SIAM Rev. 1981. V. 23. P. 308-345.

[77] Nayfeh A. H. Triple deck structure // Comput. \& Fluids. 1991. V. 20. P. 269-292.

[78] Meyer R. E. A view of the triple deck // SIAM J. Appl. Math. 1983. V. 43. P. 639-663.

[79] Zeytounian R. Kh. Meteorological Fluid Dynamics. Lecture Notes in Phys. V. 5. Berlin: Springer-Verlag, 1991.

[80] Heywood J. G. Open problems in the theory of the Navier-Stokes equations for viscous incompressible flow // Lecture Notes in Math. 1989. V. 1431. P. 1-22.

[81] Berger S. A. Initial-value stability analysis of a liquid jet // SIAM J. Appl. Math. 1988. V. 48. № 5. P. 973-991.

[82] Sulem C., Sulem P. L. The well-posedness of two-dimensional ideal flow // J. Méc. Théor. Appl. Suppl. 1983. P. 217-242.

[83] Moore D. W. The spontaneous appearance of a singularity in the shape of an evolution vortex sheet // Proc. Roy. Soc. London Ser. A. 1979. V. 365. P. 105-119.

[84] Guiraud J.-P., Zeytounian R. Kh. Note sur un mécanisme d'instabilité pour le coeur d'une nappe tourbillonnaire enroulée // Rech. Aérospat. 1979. V. 2 (Mars-Avril). P. 85-88.

[85] Bardos C. Nonlinear Euler equations and Burgers equation. Relation with Turbulence // Nonlinear Partial Differential Equations and Applications. Proceedings, Indiana University 1976-1977, Lecture Notes in Math. V. 648. Berlin: Springer-Verlag, 1978. P. 1-46.

[86] Эбин Д., Марсден Дж. Группы диффеоморфизмов и движение несжимаемой жидкости // Математика (сборник переводов). 1973. Т. 17. № 5. С. 142-167; №6. С. 111-146. 
[87] Sulem C., Sulem P. L., Bardos C., Frisch U. Finite time analyticity for the twoand three dimensional Kelvin-Helmholtz instability // Comm. Math. Phys. 1981. V. 80. P. $769-790$.

[88] Зейтунян Р. Х. Нелинейные длинные волны на поверхности воды и солитоны // УФН. 1995. Т. 165. № 12. С. 1403-1456.

[89] Уизем Дж. Линейные и нелинейные волны. М.: Мир, 1977.

[90] Zeytounian R. Kh. A quasi-one-dimensional asymptotic theory for non-linear water waves // J. Engrg. Math. 1994. V. 28. P. 261-296.

[91] Sulem C., Sulem P. L., Bardos C., Frisch U. Finite time analyticity for the twoand three-dimensional Kelvin-Helmholtz instability // Preprint Université de Nice, Dept. de Mathématiques, November, 1980.

[92] Nayfeh A.H., Saric W.S. Nonlinear Kelvin-Helmholtz instability // J. Fluid Mech. 1971. V. 46. P. 209-231.

[93] Nayfeh A.H., Saric W.S. Nonlinear waves in a Kelvin-Helmholtz flow // J. Fluid Mech. 1972. V. 55. P. 311-327.

[94] Weissman M. A. Nonlinear wave packets in the Kelvin-Helmholtz instability // Philos. Trans. Roy. Soc. London. Ser. A. 1979. V. 290. № 1377. P. 639-685.

[95] Биркгоф Г., Сарантонелло Э. Струи, следы и каверны. М.: Мир, 1964.

[96] Олейник О.А. О системе уравнений в теории пограничного слоя // Журн. вычисл. матем. и матем. физики. 1963. Т. 3. №3. С. 489-507.

[97] Nickel K. Prandtl's boundary-layer theory from the viewpoint of a mathematician // Annual Rev. Fluid Mech. 1973. V. 5. P. 405-428.

[98] Goldstein S. On laminar boundary layer flow near a point of separation // Quart. J. Mech. Appl. Math. 1948. V. 1. P. 43-69.

[99] Stewartson K. Is the singularity at separation removable? // J. Fluid Mech. 1970. V. 44. P. 347-364.

[100] Сычев В.В. О ламинарном отрыве // Изв. АН СССР. Мех. жидк. и газа. 1972. Т. 3. C. $47-59$.

[101] Catherall D., Mangler K. W. The integration of the two-dimensional laminar boundary-layer equations past the point of vanishing skin friction // J. Fluid Mech. 1966. V. 26. P. $163-182$.

[102] Нейланд В. Я. К теории отрьва ламинарного пограничного слоя в сверхзвуковом потоке // Изв. АН СССР. Мех. жидк. и газа. 1969. Т. 4. С. 53-57.

[103] Stewartson K., Williams P. G. Self-induced separation // Proc. Roy. Soc. London. Ser. A. 1969. V. 312. P. 181-206.

[104] Mauss J. Asymptotic modelling for separating boundary layers // Lecture Notes in Phys. 1995. V. 44. P. 239-254.

[105] Mangler K. W., Smith J. H. B. Behaviour of the vortex sheet at trailing edge of a lifting wing // Aero. J. Roy. Aeron. Soc. 1970. V. 74. P. 906-908.

[106] Smith F. T. The laminar separation of an incompressible fluid streaming past a smooth surface // Proc. Roy. Soc. London. Ser. A. 1977. V. 356. P. 433-463.

[107] Guiraud J.-P., Zeytounian R. Kh. Séparation d'une nappe tourbillonnaire sur une surface régulière à grand nombre de Reynolds // Journal de Mécanique. 1979. V. 18. № 3 . P. 423-431.

[108] Smith F. T. On the high Reynolds number theory of laminar flows // IMA J. Appl. Math. 1982. V. 28. P. 207-281.

[109] Сычев В.В.Асимптотическая теория отрывных течений. М.: Наука, 1987.

[110] Cercignani C. Theory and Application of the Boltzmann Equation. Edinburgh: Scottish Academic Press, 1975.

[111] Гринспен Х. Теория вращающихся жидкостей. Л.: Гидрометеоиздат, 1975.

[112] Okazawa N. The Euler equation on a bounded domain as a quasilinear evolution equation // Comm. Appl. Nonlinear Anal. 1996. V. 3. № 3. P. 107-113.

[113] Okazawa N., Unai A. Abstract quasilinear evolution equations in a Hilbert space, with applications to symmetric hyperbolic systems // SUT J. Math. 1993. V. 29. № 2. P. 263-290. 
[114] Ghidaglia J. M. Regularité des solutions de certains problemes aux limites linéaires liés aux équations d'Euler // Comm. Partial Differential Equations. 1984. V. 9. P. 1265-1298.

[115] Benedetto D., Marchioro C., Pulvirenti M. On the Euler flow in $\mathbb{R}^{2} / /$ Arch. Rational Mech. Anal. 1993. V. 123. № 4. P. 377-386.

[116] Lions P.-L., Perthame B., Souganidis P. E. Existence and stability of entropy solutions for the hyperbolic systems of isentropic gas dynamics in Eulerian and Lagrangian coordinates // Comm. Pure Appl. Math. 1996. V. 49. №6. P. 599-638.

[117] Di Perna R. J. Convergence of the viscosity method for isentropic gas dynamics // Comm. Math. Phys. 1983. V. 91. P. 1-30.

[118] Di Perna R.J. Convergence of approximate solutions to conservation laws // Arch. Rational Mech. Anal. 1983. V. 82. P. 27-70.

[119] Brenier Y. On the motion of an ideal incompressible fluid // Partial Differential Equations of Elliptic Type / ed. A. Alvino. Proceedings of the conference held on October 12-16, 1992, in Cortona, Italy, Sympos. Math. V. 35. Cambridge: Cambridge Univ. Press, 1994. P. 123-148.

[120] Constantin P., Weinan E., Titi E. S. Onsager's conjecture on the energy conservation for solutions of Euler's equation // Comm. Math. Phys. 1994. V. 165. № 1. P. 207-209.

[121] Onsager L. // Nuovo Cimento Suppl. Al. Ser. IX. 1949. V. 6. № 2. P. 279-287.

[122] Arendt S. On the non-existence of steady confined flows of a barotropic fluid in a gravitational field // J. Fluid Mech. 1994. V. 271. P. 341-350.

[123] Блохин А. М., Биркин А. Д. Глобальная разрешимость задачи о сверхзвуковом обтекании конуса // Матем. моделир. 1996. Т. 8. № 4. С. 89-104.

[124] Benney D. J. Significant interactions between small and large scale surface waves // Stud. Appl. Math. 1976. V. 55. P. 93-106.

[125] Tsutsumi M., Hatano H. Well-posedness of the Cauchy problem for the long wave-short wave resonance equations // Nonlinear Anal. 1994. V. 22. № 2. P. 155-171.

[126] Constantin P. A few results and open problems regarding incompressible fluids // Notices Amer. Math. Soc. 1995. V. 42. №6. P. 658-663.

[127] Ben-Artzi M. Global solutions of two-dimensional Navier-Stokes and Euler equations // Arch. Rational Mech. Anal. 1994. V. 128. № 4. P. 329-358.

[128] Brezis H. Remarks on the preceding paper by M. Ben-Artzi "Global solutions of two-dimensional Navier-Stokes and Euler equations" // Arch. Rational Mech. Anal. 1994. V. 128. № 4. P. 359-360.

[129] Galdi G. P., Sohr H. On the asymptotic structure of plane steady flow of a viscous fluid in exterior domains // Arch. Rational Mech. Anal. 1995. V. 131. № 2. P. 101-119.

[130] Galdi G. P. Existence and uniqueness at low Reynolds number of stationary plane flow of a viscous fluid in exterior domains // Recent Developments in Theoretical Fluid Mechanics. Papers presented at the 2nd winter school in fluid dynamics, held in Paseky, Czech Republic, in November 29-December 4, 1992 / ed. G. P. Galdi and J. Necas. Harlow: Pitman, 1993. P. 1-33.

[131] Amick C. J. On Leray's problem of steady Navier-Stokes flow past a body in the plane // Acta Math. 1988. V. 161. №1/2. P. 71-130.

[132] Galdi G. P. An Introduction to the Mathematical Theory of the Navier-Stokes equations. I. Linearized Steady Problems. New York: Springer-Verlag, 1994.

[133] Galdi G. P. An Introduction to the Mathematical Theory of the Navier-Stokes equations. II. Nonlinear Steady Problems. New York: Springer-Verlag, 1994.

[134] Amann H. Stability of the rest state of a viscous incompressible fluid // Arch. Rational Mech. Anal. 1994. V. 126. № 3. P. 231-242.

[135] Kozono H., Ogawa T. Decay properties of strong solutions for the Navier-Stokes equations in two-dimensional unbounded domains // Arch. Rational Mech. Anal. 1993. V. 122. № 1. P. 1-17.

[136] Frehse J., Rùzicka M. Regularity for the stationary Navier-Stokes equations in bounded domains // Arch. Rational Mech. Anal. 1994. V. 128. № 4. P. 361-380. 
[137] Tani A., Tanaka N. Large-time existence of surface waves in incompressible viscous fluids with or without surface tension // Arch. Rational Mech. Anal. 1995. V. 130. № 4 . P. 303-314.

[138] Tani A. Small-time existence for the three-dimensional Navier-Stokes equations for an incompressible fluid with a free surface // Arch. Rational Mech. Anal. 1996. V. 133. № 4 . P. 299-331.

[139] Елизарова Т. Г., Четверушкина Б. Н. Математическое моделирование процессов в нелинейных средах. М.: Наука, 1986.

[140] Шеретов Ю. В. Теорема о диссипации энергии и точные решения системы квазигидродинамических уравнений // Журн. вычисл. матем. и матем. физики. 1994. Т. 34. № 3. C. $483-491$.

[141] Novotny A., Padula M. $L^{p}$-approach to steady flows of viscous compressible fluids in exterior domains // Arch. Rational Mech. Anal. 1994. V. 126. № 3. P. 243-297.

[142] Beirão da Veiga H. An $L^{p}$-theory for the $n$-dimensional, stationary, compressible Navier-Stokes equations, and the incompressible limit for compressible fluids. The equilibrium solutions // Comm. Math. Phys. 1987. V. 109. P. 229-248.

[143] Farwig R. Stationary solutions of compressible Navier-Stokes equations with slip boundary condition // Comm. Partial Differential Equations. 1989. V. 14. № 11. P. 1579-1606.

[144] Padula M. On the exterior steady problem for the equations of a viscous isothermal gas // Comment. Math. Univ. Carolin. 1993. V. 34. № 2. P. 275-293.

[145] Valli A. On the existence of stationary solutions to compressible Navier-Stokes equations // Ann. Inst. H. Poincaré. Anal. Non Linéaire. 1987. V. 4. P. 99-113.

[146] Padula M. A representation formula for the steady solutions of a compressible fluid moving at low speed // Transport Theory Statist. Phys. 1992. V. 21. № 4-6. P. 593-613.

[147] Beirão da Veiga H. Singular limits in compressible fluid dynamics // Arch. Rational Mech. Anal. 1994. V. 128. № 4. P. 313-327.

[148] Zeytounian R. Kh., Guiraud J.-P. Asymptotic features of low Mach number flows in aerodynamics and in the atmosphere // Advances in Computational Methods for Boundary and Interior Layers / ed. J. J. H. Miller. Dublin: Boole Press, 1984. P. 95-100.

[149] Nordström J. The use of characteristic boundary conditions for the Navier-Stokes equations // Comput. \& Fluids. 1995. V. 24. №5. P. 609-623.

[150] Kozono H., Ogawa T. On stability of Navier-Stokes flows in exterior domains // Arch. Rational Mech. Anal. 1994. V. 128. № 1. P. 1-31.

[151] von Wahl W. Necessary and sufficient conditions for the stability of flows of incompressible viscous fluids // Arch. Rational Mech. Anal. 1994. V. 126. № 2. P. 103-129.

[152] Sattinger D. H. The mathematical problem of hydrodynamic stability // J. Math. Mech. 1970. V. 19. P. 797-817.

[153] Galdi G. P., Padula M. A new approach to energy theory in the stability of fluid motion // Arch. Rational Mech. Anal. 1990. V. 110. № 3. P. 187-286.

[154] Brevdo L. Initial-boundary value stability problem for the Blasius boundary layer // Z. Angew. Math. Mech. 1995. V. 75. № 5. P. 371-378.

[155] Alekseev V.A. Application of optimal control methods for constructing finite difference schemes in hydrodynamics // Russian J. Numer. Anal. Math. Modelling. 1994. V. 9. № 4. P. $315-336$.

[156] Szepessy A., Xin Z.P. Nonlinear stability of viscous shock waves // Arch. Rational Mech. Anal. 1993. V. 122. №1. P. 53-103.

[157] Liu T.-P. Nonlinear Stability of Shock Waves for Viscous Conservation Laws. Providence, RI: Amer. Math. Soc., 1985. (Mem. Amer. Math. Soc. V. 328.)

[158] Liu T.-P. Shock waves for compressible Navier-Stokes equations are stable // Comm. Pure Appl. Math. 1986. V. 39. P. 565-594.

[159] Bridges T. J., Mielke A. A proof of the Benjamin-Feir instability // Arch. Rational Mech. Anal. 1995. V. 133. № 2. P. 145-198.

[160] Benjamin T. B., Feir J. E. The disintegration of wave trains on deep water. I: Theory // J. Fluid Mech. 1967. V. 27. P. 417-430. 
[161] Groves M. D. A new Hamiltonian formulation of the steady water-wave problem // Structure and Dynamics of Nonlinear Waves in Fluids / ed. A. Mielke, K. Kirchgässner. Singapore: World Scientific, 1995. P. 259-267.

[162] de Conink F., Guiraud J.-P., Zeytounian R. Kh. A short look at nonlinear hydrodynamic stability theory // Quart. J. Mech. Appl. Math. 1983. V. 36. №1. P. 1-18.

[163] Afendikov A., Mielke A. Bifurcations of Poiseuille flow between parallel plates: three-dimensional solutions with large spanwise wavelength // Arch. Rational Mech. Anal. 1995. V. 129. № 2. P. 101-127.

[164] Lions P.-L. Compacité des solutions de Navier-Stokes compressibles isentropiques // C. R. Acad. Sci. Paris. Sér. I Math. 1993. V. 317. P. 115-120.

[165] Craig W., Sulem C., Sulem P. L. Nonlinear modulation of gravity waves: a rigorous approach // Nonlinearity. 1992. V. 5. P. 497-522.

[166] Padula M., Petunin I. Asymptotic behaviour of solutions to the exterior problem for the Oberbeck-Boussinesq equations // European. J. Mech. B Fluids. 1994. V. 13. №6. P. 701-730.

[167] Shen S. S. P. On the accuracy of the stationary forced Korteweg-de Vries equation as a model equation // Quart. Appl. Math. 1995. V. 53. № 4. P. 701-719.

[168] Глушко А. В., Глушко Е. В. Асимптотическое поведение при $t \rightarrow \infty$ решения задачи Коши для динамики теплопроводящей жидкости // Журн. вычисл. матем. и матем. физики. 1995. Т. 35. № 8. C. 1271-1277.

[169] Bernardi C., Metivet B., Pernaud-Thomas B. Couplage des équations de Navier-Stokes et de la chaleur: le modèle et son approximation par éléments finis // RAIRO Modél. Math. Anal. Numér. 1995. V. 29. № 7. P. 871-921.

[170] Secchi P. Well-posedness of characteristic symmetric hyperbolic systems // Arch. Rational Mech. Anal. 1996. V. 134. №2. P. 155-197.

[171] Szepessy A., Zumbrun K. Stability of rarefaction waves in viscous media // Arch. Rational Mech. Anal. 1996. V. 133. № 3. P. 249-298.

[172] Лоренц Э.Н.Детерминированное непериодическое течение // Странные аттракторы. М.: Мир, 1981. С. 88-116.

[173] Huerre P. Spatio-temporal instabilities in closed and open flows // Instabilities and Nonequilibrium Structures / ed. E. Trirapegui, D. Villarroel. Dordrecht: Reidel, 1987. P. $141-177$.

[174] Ландау Л.Д. К проблеме турбулентности // Докл. АН СССР. 1944. Т. 44.№8. C. $339-342$.

[175] Hopf E. A mathematical example displaying features of turbulence // Comm. Pure Appl. Math. 1948. V. 1. P. 303-322.

[176] Синай Я. Г., Шильников Л. П. (ред. ) Странные аттракторы. М.: Мир, 1981.

[177] Рюэль Д., Такенс А. О природе турбулентности // Странные аттракторы. М.: Мир, 1981. C. $117-151$.

[178] Ruelle D., Takens F. Note concerning our paper "On the nature of turbulence" // Comm. Math. Phys. 1971. V. 23. P. 343-344.

[179] Errafiy M., Zeytounian R. Kh. The Bénard problem for deep convection: Routes to chaos // Internat. J. Engrg. Sci. 1991. V. 29. № 11. P. 1363-1373.

[180] Feigenbaum M. J. Quantitative universality for a class of nonlinear transformations // J. Statist. Phys. 1978. V. 19. № 1. P. 25-52.

[181] Tresser C., Coullet P. H. Iterations d'endomorphismes et groupe de renormalisation // C. R. Acad. Sci. Paris. Sér. I Math. 1978. V. 287. P. 577-580.

[182] Pomeau Y., Manneville P. Intermittent transition to turbulence in dissipative dynamical systems // Comm. Math. Phys. 1980. V. 77. P. 189-197.

[183] Swinney H. L. Observations of order and chaos in nonlinear systems // Physica D. Nonlinear Phenomena. 1983. V. 7. №1-3. P. 3-15.

[184] Guckenheimer J. Strange attractors in fluids: Another view // Annual Rev. Fluid Mech. 1986. V. 18. P. 15-31. 
[185] Ladyzhenskaya O. A. Regularity of the generalized solutions of the general nonlinear and nonstationary Navier-Stokes equations // The Mathematical Problems in Fluid Mechanics. Warszawa: Institute of Basic Technical Problem, Polish Acad. of Sci., 1967. P. 61-86.

[186] Thual O. Generic instabilities and nonlinear dynamics // Astrophysical Fluid Dynamics / ed. J.P. Zahn, J. Zinn-Justin. Amsterdam: Elsevier Sci. Publ. B. V., 1988; // см. также: Dynamique des Fluides Astrophysiques. Comptes Rendues de la Session 47, Les Houches, 29 Juin-31 Juillet 1987. Amsterdam: North-Holland, 1993. P. 93-138.

[187] Guiraud J.-P. Effets non-linéaires en théorie de la stabilité des écoulements laminaires // Ann. Phys. France. 1980. V. 5. P. 33-59.

[188] Guiraud J.-P., Iooss G. Sur la stabilité des écoulements laminaires // C. R. Acad. Sci. Paris. Sér. I Math. 1968. V. 266. P. 1283-1286.

[189] Шустер Г. Г. Детерминированный хаос: введение. М.: Мир, 1988.

[190] Peixoto M. Structural stability on two-dimensional manifolds // Topology. 1962. V. 1. P. $101-120$.

[191] Eckmann J. P. Roads to turbulence in dissipative dynamical systems // Rev. Modern Phys. 1981. V. 53. № 4. P. 643-654.

[192] Newhouse S., Ruelle D., Takens F. Occurrence of strange Axiom A attractors near quasiperiodic flows on $T^{m}, m \geqslant 3 / /$ Comm. Math. Phys. 1978. V. 64. P. 35-40.

[193] Feigenbaum M. J. Universal behaviour in nonlinear systems // Los Alamos Science. 1980. V. 1. № 2. 4-27. (Перепечатано в журнале: Physica D. Nonlinear Phenomena. V. 7. № 1-3. Р. 16-39. Прим. перев.)

[194] Iooss G., Peyret R. (ed. ) Bifurcations and Chaotic Behaviors // J. Méc. Théor. Appl. Suppl. 1984.

[195] Manneville P., Pomeau Y. Intermittency and the Lorenz model // Phys. Lett. A. 1979. V. 75. № 1-2. P. 1-2.

[196] Iooss G., Joseph D.D. Elementary Stability and Bifurcation Theory. New York: Springer-Verlag, 1980.

[197] Iooss G. Bifurcation and transition to turbulence in hydrodynamics // CIME Session on Bifurcation Theory and Applications / ed. L. Salvadori. Lecture Notes Math. V. 1057. Berlin: Springer-Verlag, 1984. P. 152-201.

[198] Вайнберг М. М., Треногин В.А.Методы Ляпунова и Шмидта в теории нелинейных уравнений и их дальнейшее развитие // УМН. 1962. Т. 17. № 2. С. 13-75.

[199] Guckenheimer J., Holmes Ph. Nonlinear Oscillations, Dynamical Systems and Bifurcations of Vector Fields. New York: Springer-Verlag, 1983.

[200] Coullet P. H., Spiegel E. A. Amplitude equations for systems with competing instabilities // SIAM J. Appl. Math. 1983. V. 43. № 4. P. 776-821.

[201] Barenblatt G.I., Iooss G., Joseph D. D. (ed. ) Nonlinear Dynamics and Turbulence. Boston: Pitman, 1983.

[202] Zeeman E.C. Bifurcation, catastrophe, and turbulence // New Directions in Applied Mathematics / ed. P. J. Hilton, G.S. Young. New York: Springer-Verlag, 1982. P. 109-153.

[203] Swinney H.L., Gollub J.P. The transition to turbulence // Physics Today. 1978. V. 31. № 8. P. 41-49.

[204] Арнольд В. И. Теория катастроф. М.: Наука, 1990.

[205] Abraham R., Shaw C. D. Dynamics. The geometry of behavior. Part 2. Chaotic Behavior. Santa Cruz, California: Aerial Press Inc., 1984; Второе издание: Dynamics. The geometry of behavior. Part 1: Periodic behavior. Part 2: Chaotic behavior. Part 3: Global behavior. Part 4: Bifurcation behavior. Redwood City, CA: Addison-Wesley, 1992.

[206] Foias C., Manley O., Temam R. Sur l'interaction des petits et grands tourbillons dans des écoulements turbulents // C. R. Acad. Sci. Paris. Sér. I Math. 1987. V. 305. P. 497-500.

[207] Temam R. Attractors for the Navier-Stokes equations. Localization and approximation // J. Fac. Sci. Univ. Tokyo, Sect. I A. 1989. V. 36. № 3. P. 629-647.

[208] Foias C., Temam R. Approximation of attractors by algebraic or analytic sets // SIAM J. Math. Anal. 1994. V. 25. № 5. P. 1269-1302.

[209] Mandelbrot B. The Fractal Geometry of Nature. San Francisco: Freeman, 1982. 
[210] Chepyzhov V.V., Vishik M. I. Attractors of non-autonomous dynamical systems and their dimensions // J. Math. Pures Appl. (9). 1994. V. 73. № 3. P. 279-333.

[211] Temam R. Infinite Dimensional Dynamical Systems in Mechanics and Physics. Appl. Math. Sci. V. 68. New York: Springer-Verlag, 1988.

[212] Канторович Л. В., Крылов В. И. Приближенные методы высшего анализа. М.-Л.: Гостехиздат, 1952.

[213] Foias C., Saut J. C. Nonlinear spectral manifolds for the Navier-Stokes equations // Proc. Sympos. Pure Math. 1986. V. 45. № 1. P. 439-448.

[214] Ghidaglia J.-M. Some backward uniqueness results // Nonlinear Anal. 1986. V. 10. № 8. P. 777-790.

[215] Temam R. The Navier-Stokes Equations and Nonlinear Functional Analysis. CBMS NSF Regional Conference Series in Applied Mathematics. V. 41. Philadelphia, PA: SIAM, 1983.

[216] Doering C. R., Gibbon J. D. Applied Analysis of the Navier-Stokes Equations. Cambridge: Cambridge Univ. Press, 1995.

[217] Troshkin O. V. Nontraditional Methods in Mathematical Hydrodynamics. Transl. Math. Monographs. V. 144. Providence, RI: Amer. Math. Soc., 1995.

[218] Kobayashi R. (ed. ) Laminar-Turbulent Transition. Berlin: Springer-Verlag, 1995.

[219] Coweley S. J., Wu X. Asymptotic approaches to transition modelling // Progress in Transition Modelling. AGARD Rep. V. 793, 1994, Ch. 3. P. 1-38.

[220] Daveney R.L., Keen L. (ed. ) Chaos and Fractals: The Mathematics Behind the Computer Graphics // Proc. Sympos. Appl. Math. 1989. V. 39.

[221] Baker G. L., Gollub J. P. Chaotic dynamics. An introduction. Cambridge: Cambridge Univ. Press, 1996.

[222] Aref H., El Naschie M.S. (ed. ) Chaos Applied to Fluid Mixing. Oxford: Pergamon, 1995. (Reprinted from the journal: Chaos, Solitons and Fractals. V. 4. № 6, 1994.)

[223] Beck C., Schlögl F. Thermodynamics of Chaotic Systems. An Introduction. Cambridge Nonlinear Science Series. V. 4. Cambridge: Cambridge Univ. Press, 1995.

[224] Marek M., Schreiber I. Chaotic Behaviour of Deterministic Dissipative Systems. Cambridge: Cambridge Univ. Press, 1995.

[225] Godreche C., Manneville P. Hydrodynamics and Nonlinear Instabilities. Collection Alea-Saclay: Monographs and Texts in Statistical Physics. Cambridge: Cambridge Univ. Press, 1995.

[226] van Groesen E., Jager E. M. Mathematical Structures in Continuous Dynamical Systems. Poisson Systems and Complete Integrability with Applications from Fluid Dynamics. Studies in Mathematical Physics. V. 6. Amsterdam: North-Holland, 1994.

[227] Nicolis G. Introduction to Nonlinear Science. Cambridge: Cambridge Univ. Press, 1995.

[228] Kevorkian J., Cole J. D. Multiple Scale and Singular Perturbation Methods. Applied Mathematical Sciences. V. 114. New York: Springer-Verlag, 1996. 